Stellarators close to quasisymmetry

This content has been downloaded from IOPscience. Please scroll down to see the full text. 2013 Plasma Phys. Control. Fusion 55125014

(http://iopscience.iop.org/0741-3335/55/12/125014)

View the table of contents for this issue, or go to the journal homepage for more

Download details:

IP Address: 130.206.40.7

This content was downloaded on 20/11/2013 at 15:07

Please note that terms and conditions apply. 


\title{
Stellarators close to quasisymmetry
}

\author{
Iván Calvo $^{1}$, Felix I Parra ${ }^{2}$, José Luis Velasco ${ }^{1}$ and J Arturo Alonso ${ }^{1}$ \\ ${ }^{1}$ Laboratorio Nacional de Fusión, Asociación EURATOM-CIEMAT, 28040 Madrid, Spain \\ 2 Plasma Science and Fusion Center, MIT, Cambridge, MA 02139, USA \\ E-mail: ivan.calvo@ciemat.es, fparra@mit.edu, joseluis.velasco@ciemat.es and \\ arturo.alonso@ciemat.es
}

Received 12 July 2013, in final form 24 September 2013

Published 20 November 2013

Online at stacks.iop.org/PPCF/55/125014

\begin{abstract}
Rotation is favorable for confinement, but a stellarator can rotate at high speeds if and only if it is sufficiently close to quasisymmetry. This article investigates how close it needs to be. For a magnetic field $\boldsymbol{B}=\boldsymbol{B}_{0}+\alpha \boldsymbol{B}_{1}$, where $\boldsymbol{B}_{0}$ is quasisymmetric, $\alpha \boldsymbol{B}_{1}$ is a deviation from quasisymmetry, and $\alpha \ll 1$, the stellarator can rotate at high velocities if $\alpha<\epsilon^{1 / 2}$, with $\epsilon$ the ion Larmor radius over the characteristic variation length of $\boldsymbol{B}_{0}$. The cases in which this result may break down are discussed. If the stellarator is sufficiently quasisymmetric in the above sense, the rotation profile, and equivalently, the long-wavelength radial electric field, are not set neoclassically; instead, they can be affected by turbulent transport. Their computation requires the $O\left(\epsilon^{2}\right)$ pieces of both the turbulent and the long-wavelength components of the distribution function. This article contains the first step towards a formulation to calculate the rotation profile by providing the equations determining the long-wavelength components of the $O\left(\epsilon^{2}\right)$ pieces.
\end{abstract}

(Some figures may appear in colour only in the online journal)

\section{Introduction}

In a stellarator, quasisymmetry and the capability to sustain large rotation are equivalent $[1,2]$. This means that only when the stellarator is quasisymmetric, there exists a component of the flow (the component in the symmetry direction) on the flux surface that is neoclassically undamped. For design purposes, large rotation only requires that the stellarator be sufficiently close to quasisymmetry, as is physically reasonable. This is important because, on the one hand, large rotation is beneficial for magnetohydrodynamic modes and confinement [3], and therefore desirable; on the other hand, Garren and Boozer proved [4] that except for the axisymmetric case, quasisymmetric toroidal magnetic fields do not exist ${ }^{3}$. Then, it is natural to ask what is the maximum size of a deviation from quasisymmetry that still allows sonic rotation speeds. Let us write the magnetic field as $\boldsymbol{B}=\boldsymbol{B}_{0}+\alpha \boldsymbol{B}_{1}$, where $\boldsymbol{B}_{0}$ is quasisymmetric, $\alpha \ll 1$, and $\alpha \boldsymbol{B}_{1}$ represents a deviation from quasisymmetry. Reference [4] proved that $\alpha=0$ cannot be achieved. In this paper, and in the framework of gyrokinetic theory, we find that in general a stellarator

3 Garren and Boozer proved that quasisymmetry is possible on one flux surface but not throughout the entire plasma volume. plasma can rotate fast if

$$
\alpha<\epsilon^{1 / 2} \text {. }
$$

Here, $\epsilon:=\rho_{\mathrm{i}} / L$, where $\rho_{\mathrm{i}}$ is a typical ion Larmor radius and $L$ is the characteristic variation length of $\boldsymbol{B}_{0}$. This scaling was already obtained in a fluid description of a highly collisional plasma in [5]. Advancing the results of section 7, we point out that (1) is the correct criterion to evaluate closeness to quasisymmetry as long as the helicity of $\boldsymbol{B}_{1}$ is small enough.

Whether the deviation from quasisymmetry satisfies (1) or not has consequences for the theoretical computation of the rotation profile or, equivalently, the long-wavelength radial electric field. If (1) is not satisfied, the problem is neoclassical. If (1) holds, the effect of microturbulent fluctuations has to be taken into account. Let us explain the problem in detail.

Only in recent years has the problem of the determination of the long-wavelength radial electric field in a turbulent tokamak in the low flow ordering (i.e. with subsonic speeds) been understood [6-8]. First, it has been shown that computing the long-wavelength radial electric field is equivalent to the calculation of the toroidal momentum transport or to the determination of the toroidal rotation profile. The momentum flux in tokamaks is mostly due to microturbulence, well 
described by gyrokinetic theory. It requires gyrokinetic equations that are accurate to $O\left(\epsilon^{2}\right)$, because the expressions for obtaining the toroidal rotation profile involve the $O\left(\epsilon^{2}\right)$ pieces of the distribution function (the turbulent and longwavelength components) and some $O\left(\epsilon^{2}\right)$ pieces of the electrostatic potential (the complete turbulent component and the part corresponding to the long-wavelength poloidal electric field). An introductory overview of the problem is given in [9]. The derivation of the electrostatic gyrokinetic equations to $O\left(\epsilon^{2}\right)$ in general magnetic geometry has been carried out in [10]. A complete set of equations to find the tokamak long-wavelength radial electric field has been given [11-13] that is valid when $B / B_{\mathrm{p}} \gg 1$, where $B$ is the modulus of the magnetic field and $B_{\mathrm{p}}$ is the modulus of the poloidal magnetic field. The general proof that the turbulent tokamak is intrinsically ambipolar and the subset of long-wavelength equations that will eventually be needed to correctly compute toroidal momentum transport in a tokamak, without relying on the assumption $B / B_{\mathrm{p}} \gg 1$, have been given in [7,14].

The origin of the difficulty is the intrinsic ambipolarity of the tokamak, even in the presence of turbulence; that is, the fact that to lowest order the flux-surface averaged radial flux of charge is identically zero, whatever the value of the long-wavelength radial electric field. This is the property that allows the tokamak to rotate. Intrinsic ambipolarity in the tokamak implies that the flow in the toroidal direction is neoclassically undamped, that the plasma can rotate freely, and that the long-wavelength radial electric field is not determined by the lowest-order calculation in $\epsilon$. The theoretical calculation of the long-wavelength radial electric field in a generic stellarator is comparatively much easier because it is determined by neoclassical physics due to the lack of intrinsic ambipolarity: parallel viscosity damps the two components of the flow on the flux surface, setting the longwavelength radial electric field. This is true even for some types of stellarators optimized with respect to neoclassical transport. For example, collisionless particle trajectories in omnigeneous [15-17] and isodynamic stellarators [18] have vanishing average radial velocity but the radial particle fluxes need not be intrinsically ambipolar, and the radial electric field is computed from the standard neoclassical ambipolarity condition. The most constrained stellarator design concept is quasisymmetry $[19,20]$. Quasisymmetric stellarators are omnigenous and isodynamic, but the converse is not true. Since a stellarator is quasisymmetric if and only if it is intrinsically ambipolar [1,2], it is expected that the problem of solving for the radial electric field in these devices be similar to the one in the tokamak. The same can be said for the computation of the rotation profile along the direction of symmetry of the quasisymmetric stellarator.

Although the general picture is analogous in quasisymmetric stellarators and in tokamaks, the former exhibit very interesting subtleties [21,22]. In particular, a remark in connection with the striking result of [22] is in order. In this reference it was found that stellarators, even if quasisymmetric, cannot rotate to speeds that are strictly sonic; only the axisymmetric tokamak can. However, the result is not as negative for rotation in stellarators as this statement might suggest. Let us denote by $M=V_{\mathrm{i}} / v_{\mathrm{ti}}$ the Mach number, where $V_{\mathrm{i}}$ is the plasma rotation speed and $v_{\mathrm{ti}}$ is the ion thermal speed. Typically, there is much room between $\epsilon v_{\mathrm{ti}}$ and $v_{\mathrm{ti}}$. If $\epsilon v_{\mathrm{ti}} \ll M v_{\mathrm{ti}} \ll v_{\mathrm{ti}}$, then a subsidiary expansion in the Mach number is possible and the mathematical obstructions for rotation found in [22] disappear. It is in this setting that our work should be understood.

The problem of writing the equations that would determine the long-wavelength radial electric field, or the rotation profile, in a quasisymmetric stellarator (actually, in a stellarator satisfiying equation (1)) has never been considered. We take the first step towards a formulation that unifies the non-quasisymmetric and quasisymmetric cases. Such a formulation has to be addressed in the framework of gyrokinetic theory and implies obtaining equations for the $O\left(\epsilon^{2}\right)$ pieces of both the turbulent and long-wavelength components of the distribution function, for the $O\left(\epsilon^{2}\right)$ pieces of the turbulent electrostatic potential, and for the $O\left(\epsilon^{2}\right)$ pieces of the long-wavelength poloidal and toroidal components of the electric field. In this paper we formulate the equations for the long-wavelength components. The longwavelength Fokker-Planck and quasineutrality equations to $O\left(\epsilon^{2}\right)$ for an arbitrary stellarator are derived, and explicit expressions are given that could eventually be implemented in computer codes. Obviously, if the stellarator is nonquasisymmetric it is not necessary to perform the calculation to $O\left(\epsilon^{2}\right)$. The proof of intrinsic ambipolarity in [14] fails for non-quasisymmetric stellarators, and the equations presented here give the neoclassical radial electric field. In the quasisymmetric case, the $O\left(\epsilon^{2}\right)$ accuracy to which the gyrokinetic Fokker-Planck and quasineutrality equations are derived in this paper is necessary; physically this implies, in particular, that both neoclassical and turbulent effects have to be included. Progress in computational stellarator optimization and design techniques [23] as well as in computer simulation of the gyrokinetic equations [24-33] make the topics of this paper relevant to present-day fusion research.

The rest of the paper is organized as follows. In section 2 we introduce the basic assumptions, mathematical tools and equations of gyrokinetics, and set the notation. We also recall the results from [10] that are needed in subsequent sections. Section 3.1 is devoted to taking the long-wavelength limit of the gyrokinetic Fokker-Planck equation to $O\left(\epsilon^{2}\right)$ by assuming only the existence of nested flux surfaces; that is, in a general stellarator. In section 3.2 we do the same for the quasineutrality equation. Although it was not emphasized there, many expressions in [14] are valid for general magnetic geometry, not only for tokamaks. Hence, much of the effort involved in the derivation of the gyrokinetic FokkerPlanck and quasineutrality equations to $O\left(\epsilon^{2}\right)$ has already been done. Accordingly, sections 3.1 and 3.2 will be shorter than the corresponding ones in [14]. Section 4 shows why the long-wavelength radial electric field is not determined, in any stellarator, by using exclusively the quasineutrality equation to second order. To find the long-wavelength radial electric field, we need the solvability conditions of the Fokker-Planck equation to order $\epsilon^{j}$, discussed in section 5. For $j=1$ it turns out that the solvability conditions are trivially satisfied, whereas for $j=2$ they 
yield transport equations for density and energy, that we give explicitly. Imposing compatibility of the density transport equation with the lowest-order quasineutrality equation leads to the well-known neoclassical ambipolarity condition, i.e. the vanishing of the lowest-order flux-surface averaged radial current. Section 6 discusses in detail when the neoclassical ambipolarity condition is not automatically satisfied, giving the long-wavelength radial electric field. In other words, we ask when the system is intrinsically ambipolar, which is equivalent to being quasisymmetric. In section 7 we discuss the violation of ambipolarity due to small deviations from quasisymmetry, determining when a stellarator can be considered quasisymmetric in practice. The conclusions are contained in section 8 .

\section{Electrostatic gyrokinetics}

The kinetic description of a plasma in a time-independent magnetic field requires the Fokker-Planck equation for each species $\sigma$,

$$
\begin{aligned}
\partial_{t} f_{\sigma} & +\boldsymbol{v} \cdot \nabla_{r} f_{\sigma}+\frac{Z_{\sigma} e}{m_{\sigma}}\left(-\nabla_{r} \varphi+c^{-1} \boldsymbol{v} \times \boldsymbol{B}\right) \cdot \nabla_{\boldsymbol{v}} f_{\sigma} \\
& =\sum_{\sigma^{\prime}} C_{\sigma \sigma^{\prime}}\left[f_{\sigma}, f_{\sigma^{\prime}}\right](\boldsymbol{r}, \boldsymbol{v}),
\end{aligned}
$$

and Poisson's equation,

$$
\nabla_{r}^{2} \varphi(\boldsymbol{r}, t)=-4 \pi e \sum_{\sigma} Z_{\sigma} \int f_{\sigma}(\boldsymbol{r}, \boldsymbol{v}, t) \mathrm{d}^{3} v .
$$

Here, $c$ is the speed of light, $e$ the charge of the proton, $\varphi(r, t)$ the electrostatic potential, $\boldsymbol{B}(\boldsymbol{r})=\nabla_{\boldsymbol{r}} \times \boldsymbol{A}(\boldsymbol{r})$ a time-independent magnetic field, $\boldsymbol{A}(\boldsymbol{r})$ the magnetic vector potential, $f_{\sigma}(\boldsymbol{r}, \boldsymbol{v}, t)$ the phase-space probability distribution, and $Z_{\sigma} e$ and $m_{\sigma}$ the charge and the mass of species $\sigma$. The Fokker-Planck collision operator between species $\sigma$ and $\sigma^{\prime}$ is

$$
\begin{aligned}
& C_{\sigma \sigma^{\prime}}\left[f_{\sigma}, f_{\sigma^{\prime}}\right](\boldsymbol{r}, \boldsymbol{v}) \\
& =\frac{\gamma_{\sigma \sigma^{\prime}}}{m_{\sigma}} \nabla_{\boldsymbol{v}} \cdot \int \stackrel{\leftrightarrow}{\boldsymbol{W}}\left(\boldsymbol{v}-\boldsymbol{v}^{\prime}\right) \cdot\left(\frac{1}{m_{\sigma}} f_{\sigma^{\prime}}\left(\boldsymbol{r}, \boldsymbol{v}^{\prime}, t\right) \nabla_{\boldsymbol{v}} f_{\sigma}(\boldsymbol{r}, \boldsymbol{v}, t)\right. \\
& \left.\quad-\frac{1}{m_{\sigma^{\prime}}} f_{\sigma}(\boldsymbol{r}, \boldsymbol{v}, t) \nabla_{\boldsymbol{v}^{\prime}} f_{\sigma^{\prime}}\left(\boldsymbol{r}, \boldsymbol{v}^{\prime}, t\right)\right) \mathrm{d}^{3} \boldsymbol{v}^{\prime}
\end{aligned}
$$

where

$$
\begin{aligned}
& \gamma_{\sigma \sigma^{\prime}}:=2 \pi Z_{\sigma}^{2} Z_{\sigma^{\prime}}^{2} e^{4} \ln \Lambda \\
& \overleftrightarrow{\boldsymbol{W}}(\boldsymbol{w}):=\frac{|\boldsymbol{w}|^{2} \stackrel{\leftrightarrow}{\boldsymbol{I}}-\boldsymbol{w} \boldsymbol{w}}{|\boldsymbol{w}|^{3}},
\end{aligned}
$$

$\ln \Lambda$ is the Coulomb logarithm, and $\stackrel{\leftrightarrow}{I}$ is the identity matrix. Some important properties of this operator are collected in appendix A. A direct check shows that the Fokker-Planck equation can also be written as

$$
\partial_{t} f_{\sigma}+\left\{f_{\sigma}, H_{\sigma}\right\}_{\boldsymbol{X}}=\sum_{\sigma^{\prime}} C_{\sigma \sigma^{\prime}}\left[f_{\sigma}, f_{\sigma^{\prime}}\right](\boldsymbol{X}),
$$

where we designate by $\boldsymbol{X} \equiv(\boldsymbol{r}, \boldsymbol{v})$ a set of euclidean coordinates in phase space,

$$
H_{\sigma}(\boldsymbol{r}, \boldsymbol{v}, t)=\frac{1}{2} m_{\sigma} \boldsymbol{v}^{2}+Z_{\sigma} e \varphi(\boldsymbol{r}, t)
$$

is the Hamiltonian of species $\sigma$, and the Poisson bracket of two functions on phase space, $g_{1}(\boldsymbol{r}, \boldsymbol{v})$ and $g_{2}(\boldsymbol{r}, \boldsymbol{v})$, is

$$
\begin{aligned}
& \left\{g_{1}, g_{2}\right\}_{\boldsymbol{X}}=\frac{1}{m_{\sigma}}\left(\nabla_{\boldsymbol{r}} g_{1} \cdot \nabla_{\boldsymbol{v}} g_{2}-\nabla_{\boldsymbol{v}} g_{1} \cdot \nabla_{\boldsymbol{r}} g_{2}\right) \\
& +\frac{Z_{\sigma} e}{m_{\sigma}^{2} c} \boldsymbol{B} \cdot\left(\nabla_{\boldsymbol{v}} g_{1} \times \nabla_{\boldsymbol{v}} g_{2}\right) .
\end{aligned}
$$

\subsection{Gyrokinetic ordering}

Relevant frequencies in magnetically confined fusion plasmas are usually much smaller than the gyrofrequency, i.e. the frequency that corresponds to the approximately circular motion of a charged particle around a magnetic field line. Gyrokinetic theory $[34,35]$ is the reduced kinetic theory obtained by averaging out the gyrofrequency time scale. This is possible due to the assumed smallness of the gyroradius, as explained in what follows.

Denoted by $L \sim\left|\nabla_{r} \ln \right| B||^{-1}$ the typical length of variation of the magnetic field and by $B_{0}$ a typical value of the magnetic field strength. The sound speed is defined as $c_{\mathrm{s}}:=\sqrt{T_{\mathrm{e} 0} / m_{\mathrm{i}}}$, where $T_{\mathrm{e} 0}$ is a typical electron temperature and $m_{\mathrm{i}}$ is the mass of the singly-charged dominant ion species. Gyrokinetics is formulated as an asymptotic expansion in the small parameter $\epsilon_{\mathrm{s}}=\rho_{\mathrm{s}} / L$, where $\rho_{\mathrm{s}}=c_{\mathrm{s}} / \Omega_{\mathrm{i}}$ is a characteristic sound gyroradius, and $\Omega_{\mathrm{i}}=e B_{0} /\left(m_{\mathrm{i}} c\right)$ is a characteristic ion gyrofrequency. Even if formally the only expansion parameter is $\epsilon_{\mathrm{s}}$, many expressions are more conveniently written in terms of the species-dependent parameter $\epsilon_{\sigma}=\rho_{\sigma} / L$, where $\rho_{\sigma}=v_{\mathrm{t} \sigma} / \Omega_{\sigma}$ is the characteristic gyroradius of species $\sigma, v_{\mathrm{t} \sigma}=\sqrt{T_{\mathrm{e} 0} / m_{\sigma}}$ is the thermal velocity, and $\Omega_{\sigma}=Z_{\sigma} e B_{0} /\left(m_{\sigma} c\right)$ is the characteristic gyrofrequency. Observe that the relation between $\epsilon_{\sigma}$ and $\epsilon_{\mathrm{s}}$ is $\epsilon_{\mathrm{s}}=\lambda_{\sigma} \epsilon_{\sigma}$, with

$$
\lambda_{\sigma}=\frac{\rho_{\mathrm{s}}}{\rho_{\sigma}}=Z_{\sigma} \sqrt{\frac{m_{\mathrm{i}}}{m_{\sigma}}} .
$$

In this paper we assume that $T_{\mathrm{e} 0}$ is the typical temperature of all species, which is justified when the time between collisions is shorter than the transport time scale. It is not difficult to relax this hypothesis. We also stress that our ordering is maximal, in the sense that typical expansions, like those in the mass ratio, can be obtained by performing subsidiary expansions in parameters such as $\lambda_{\sigma}$.

It has been experimentally observed that the characteristic correlation length of the turbulence is of the order of the gyroradius and scales with it, and that the size of the turbulent fluctuations scales with the ion gyroradius [36]. Also, the characteristic length of the turbulent eddies and the size of the fluctuations are related to each other by the background gradient. An eddy of length $\ell_{\perp} \sim \rho_{\mathrm{s}}$ mixes the plasma contained within it. In the presence of a gradient this eddy will lead to fluctuations on top of the background density of 
order $\delta n_{\mathrm{e}} \sim \ell_{\perp}\left|\nabla n_{\mathrm{e}}\right| \sim \epsilon_{\mathrm{s}} n_{\mathrm{e}} \ll n_{\mathrm{e}}$. These facts suggest the ordering that we set below.

We start by introducing a transport average, that acting on a function $g(\boldsymbol{r}, t)$ gives the component corresponding to long wavelengths and small frequencies. Let $\{\psi, \Theta, \zeta\}$ be a set of flux coordinates, where $\psi$ is the flux-surface label, $\Theta$ is the poloidal angle, and $\zeta$ is the toroidal angle. This averaging operation is

$\langle g\rangle_{\mathrm{T}}=\frac{1}{\Delta t \Delta \psi \Delta \Theta \Delta \zeta} \int_{\Delta t} \mathrm{~d} t \int_{\Delta \psi} \mathrm{d} \psi \int_{\Delta \Theta} \mathrm{d} \Theta \int_{\Delta \zeta} \mathrm{d} \zeta g$,

where $\epsilon_{\mathrm{s}} \ll \Delta \psi / \psi \ll 1, \epsilon_{\mathrm{s}} \ll \Delta \Theta \ll 1, \epsilon_{\mathrm{s}} \ll \Delta \zeta \ll 1$ and $L / c_{\mathrm{s}} \ll \Delta t \ll \tau_{E}$. Here $\tau_{E}:=\epsilon_{\mathrm{s}}^{-2} L / c_{\mathrm{s}}$ is the transport time scale. For any function $g(\boldsymbol{r}, t)$, we define

$$
\begin{gathered}
g^{\mathrm{lw}}:=\langle g\rangle_{\mathrm{T}} \\
g^{\mathrm{sw}}:=g-g^{\mathrm{lw}},
\end{gathered}
$$

which satisfies the following obvious properties:

$$
\begin{gathered}
{\left[g^{\mathrm{lw}}\right]^{1 \mathrm{w}}=g^{\mathrm{lw}},} \\
{\left[g^{\mathrm{sw}}\right]^{\mathrm{lw}}=0,} \\
{[g h]^{1 \mathrm{w}}=g^{\mathrm{lw}} h^{1 \mathrm{w}}+\left[g^{\mathrm{sw}} h^{\mathrm{sw}}\right]^{1 \mathrm{w}},}
\end{gathered}
$$

for any two functions $g(\boldsymbol{r}, t)$ and $h(\boldsymbol{r}, t)$.

We decompose the fields of our theory using the transport average:

$$
\begin{gathered}
f_{\sigma}=f_{\sigma}^{\mathrm{lw}}+f_{\sigma}^{\mathrm{sw}}, \\
\varphi=\varphi^{\mathrm{lw}}+\varphi^{\mathrm{sw}} .
\end{gathered}
$$

The length and time scales of $f_{\sigma}^{\mathrm{sw}}$ and $\varphi^{\mathrm{sw}}$ correspond to the turbulence, whereas the scales of $f_{\sigma}^{\mathrm{lw}}$ and $\varphi^{\mathrm{lw}}$ are those of the slowly varying profiles. Now, we are ready to start enumerating the ordering assumptions. The long-wavelength component of the distribution function is assumed to be larger than the shortwavelength piece by a factor of $\epsilon_{\mathrm{s}}^{-1} \gg 1$; the long-wavelength piece of the potential is itself comparable to the kinetic energy of the particles and its short-wavelength component is also small in $\epsilon_{\mathrm{s}}$, i.e.

$$
\begin{aligned}
& \frac{v_{\mathrm{t} \sigma}^{3} f_{\sigma}^{\mathrm{sw}}}{n_{\mathrm{e} 0}} \sim \frac{Z_{\sigma} e \varphi^{\mathrm{sw}}}{m_{\sigma} v_{\mathrm{t} \sigma}^{2}} \sim \epsilon_{\mathrm{s}}, \\
& \frac{v_{\mathrm{t} \sigma}^{3} f_{\sigma}^{\mathrm{lw}}}{n_{\mathrm{e} 0}} \sim \frac{Z_{\sigma} e \varphi^{\mathrm{lw}}}{m_{\sigma} v_{\mathrm{t} \sigma}^{2}} \sim 1 .
\end{aligned}
$$

Here, $n_{\mathrm{e} 0}$ is a typical electron density.

We turn to the size of the space and time derivatives of the long- and short-wavelength components of our fields. The long-wavelength components $f_{\sigma}^{\mathrm{lw}}$ and $\varphi^{\mathrm{lw}}$ are characterized by large spatial scales, of the order of the macroscopic scale $L$, and long time scales, of the order of the transport time scale, $\tau_{E}$, i.e.

$$
\begin{array}{rlrl}
\nabla_{r} \ln f_{\sigma}^{1 \mathrm{w}}, & \nabla_{\boldsymbol{r}} \ln \varphi^{1 \mathrm{w}} & \sim 1 / L, \\
\partial_{t} \ln f_{\sigma}^{\mathrm{lw}}, & \partial_{t} \ln \varphi^{\mathrm{lw}} \sim \epsilon_{\mathrm{s}}^{2} c_{\mathrm{s}} / L .
\end{array}
$$

The short-wavelength components $f_{\sigma}^{\mathrm{sw}}$ and $\varphi^{\mathrm{sw}}$ have perpendicular wavelengths of the order of the sound gyroradius, and short time scales of the order of the turbulence correlation time. The parallel correlation length of the shortwavelength component is much longer than its characteristic perpendicular wavelength, and it is comparable to the size of the machine. That is, $f_{\sigma}^{\mathrm{sw}}$ and $\varphi^{\mathrm{sw}}$ are characterized by

$$
\begin{aligned}
\hat{\boldsymbol{b}} \cdot \nabla_{\boldsymbol{r}} \ln f_{\sigma}^{\mathrm{sw},} & \hat{\boldsymbol{b}} \cdot \nabla_{\boldsymbol{r}} \ln \varphi^{\mathrm{sw}} \sim 1 / L, \\
\nabla_{\boldsymbol{r}_{\perp}} \ln f_{\sigma}^{\mathrm{sw}}, & \nabla_{\boldsymbol{r}_{\perp}} \ln \varphi^{\mathrm{sw}} \sim 1 / \rho_{\mathrm{s}}, \\
\partial_{t} \ln f_{\sigma}^{\mathrm{sw}}, & \partial_{t} \ln \varphi^{\mathrm{sw}} \sim c_{\mathrm{s}} / L,
\end{aligned}
$$

with $\hat{b}:=B / B$

The magnetic field only contains long-wavelength components,

$$
\nabla_{r} \ln |\boldsymbol{B}| \sim 1 / L .
$$

Finally, we order the collision frequency by the relation

$$
\epsilon_{\mathrm{s}} \ll v_{* \sigma \sigma^{\prime}} \ll \frac{1}{\epsilon_{\mathrm{s}}},
$$

where we are defining the collisionality as

$$
\nu_{* \sigma \sigma^{\prime}}:=L v_{\sigma \sigma^{\prime}} / v_{\mathrm{t} \sigma}
$$

and the collision frequency as

$$
v_{\sigma \sigma^{\prime}}:=\frac{4 \sqrt{2 \pi}}{3} \frac{Z_{\sigma}^{2} Z_{\sigma^{\prime}}^{2} n_{\mathrm{e} 0} e^{4}}{m_{\sigma}^{1 / 2} T_{\sigma}^{3 / 2}} \ln \Lambda,
$$

which coincides with Braginskii's definition [37] for $\sigma=e$ and $\sigma^{\prime}=i$.

The ordering introduced above leads to consistent equations to each order in $\epsilon_{\mathrm{s}}$ that capture the physics of microturbulence in strongly magnetized plasmas. This helps not only from a conceptual perspective but also, and above all, from the simulation point of view. The removal of the gyromotion time scale saves enormous computational time, allowing routine simulation of magnetized plasma turbulence [25-27, 29,38]. After introducing suitably normalized variables in section 2.2, we explain how to carry out the gyrokinetic expansion in section 2.3 .

\subsection{Dimensionless variables}

The expansion of the equations to high order in $\epsilon_{\mathrm{s}}$ is easier if we work in non-dimensionalized variables adapted to the gyrokinetic ordering [10]. We employ the species-independent normalization

$$
\begin{gathered}
\underline{t}=\frac{c_{\mathrm{s}} t}{L}, \quad \underline{\boldsymbol{r}}=\frac{\boldsymbol{r}}{L}, \quad \underline{\boldsymbol{A}}=\frac{\boldsymbol{A}}{B_{0} L}, \quad \underline{\varphi}=\frac{e \varphi}{\epsilon_{\mathrm{s}} T_{\mathrm{e} 0}}, \\
\underline{H_{\sigma}}=\frac{H_{\sigma}}{T_{\mathrm{e} 0}}, \quad \underline{n_{\sigma}}=\frac{n_{\sigma}}{n_{\mathrm{e} 0}}, \quad \underline{T_{\sigma}}=\frac{T_{\sigma}}{T_{\mathrm{e} 0}}, \quad
\end{gathered}
$$

for time, space, vector potential, electrostatic potential, Hamiltonian, particle density, and temperature; and the species-dependent normalization

$$
\underline{\boldsymbol{v}_{\sigma}}=\frac{\boldsymbol{v}_{\sigma}}{v_{\mathrm{t} \sigma}}, \quad \underline{f_{\sigma}}=\frac{v_{\mathrm{t} \sigma}^{3}}{n_{\mathrm{e} 0}} f_{\sigma},
$$

for velocities and distribution functions. 
In dimensionless variables, the Fokker-Planck equation (2) becomes

$\partial_{\underline{t}} \underline{f_{\sigma}}+\tau_{\sigma}\left\{\underline{f_{\sigma}}, \underline{H_{\sigma}}\right\}_{\underline{\boldsymbol{X}}}=\tau_{\sigma} \sum_{\sigma^{\prime}} \underline{C_{\sigma \sigma^{\prime}}}\left[\underline{f_{\sigma}}, \underline{f_{\sigma^{\prime}}}\right](\underline{\boldsymbol{r}}, \underline{\boldsymbol{v}})$,

where

$$
\tau_{\sigma}=\frac{v_{\mathrm{t} \sigma}}{c_{\mathrm{s}}}=\sqrt{\frac{m_{\mathrm{i}}}{m_{\sigma}}},
$$

and the Poisson bracket of two functions $g_{1}(\underline{\boldsymbol{r}}, \underline{\boldsymbol{v}}), g_{2}(\underline{\boldsymbol{r}}, \underline{\boldsymbol{v}}$ ) (we no longer write the subindex $\sigma$ in $\boldsymbol{v}_{\sigma}$ ) is defined by

$$
\begin{gathered}
\left\{g_{1}, g_{2}\right\}_{\underline{X}}=\left(\nabla_{\underline{\boldsymbol{r}}} g_{1} \cdot \nabla_{\underline{\boldsymbol{v}}} g_{2}-\nabla_{\underline{\boldsymbol{v}}} g_{1} \cdot \nabla_{\underline{\boldsymbol{r}}} g_{2}\right) \\
+\frac{1}{\epsilon_{\sigma}} \underline{\boldsymbol{B}} \cdot\left(\nabla_{\underline{\boldsymbol{v}}} g_{1} \times \nabla_{\underline{\boldsymbol{v}}} g_{2}\right) .
\end{gathered}
$$

Here $\underline{\boldsymbol{X}} \equiv(\underline{\boldsymbol{r}}, \underline{\boldsymbol{v}})$ are the dimensionless cartesian coordinates. The normalized collision operator is

$$
\begin{aligned}
\underline{C_{\sigma \sigma^{\prime}}} & {\left[\underline{f_{\sigma}}, \underline{f_{\sigma^{\prime}}}\right](\underline{\boldsymbol{r}}, \underline{\boldsymbol{v}})=\underline{\gamma_{\sigma \sigma^{\prime}}} \nabla_{\underline{\boldsymbol{v}}} \cdot \int \stackrel{\leftrightarrow}{\boldsymbol{W}}\left(\tau_{\sigma} \underline{\boldsymbol{v}}-\tau_{\sigma^{\prime}} \underline{\boldsymbol{v}^{\prime}}\right) } \\
& \cdot\left(\tau_{\sigma} \underline{f_{\sigma^{\prime}}}\left(\underline{\boldsymbol{r}}, \underline{\boldsymbol{v}^{\prime}}, \underline{t}\right) \nabla_{\underline{\boldsymbol{v}}} \underline{f_{\sigma}}(\underline{\boldsymbol{r}}, \underline{\boldsymbol{v}}, \underline{t})\right. \\
& \left.\left.-\tau_{\sigma^{\prime}} \underline{f_{\sigma}} \underline{(\boldsymbol{r},} \underline{\boldsymbol{v}}, \underline{t}\right) \nabla_{\underline{\underline{\boldsymbol{v}}^{\prime}}} \underline{f_{\sigma^{\prime}}}\left(\underline{\boldsymbol{r}}, \underline{\boldsymbol{v}^{\prime}}, \underline{t}\right)\right) \mathrm{d}^{3} \underline{\boldsymbol{v}^{\prime}},
\end{aligned}
$$

with

$$
\underline{\gamma_{\sigma \sigma^{\prime}}}:=\frac{2 \pi Z_{\sigma}^{2} Z_{\sigma^{\prime}}^{2} n_{\mathrm{e} 0} e^{4} L}{T_{\mathrm{e} 0}^{2}} \ln \Lambda .
$$

Observe that $\gamma_{\sigma \sigma^{\prime}}$ is the usual collisionality parameter $\nu_{* \sigma \sigma^{\prime}}$ in (20) up to a factor of order unity.

As for Poisson's equation (3),

$$
\frac{\epsilon_{\mathrm{s}} \lambda_{\mathrm{De}}^{2}}{L^{2}} \nabla_{\underline{r}}^{2} \underline{\varphi}(\underline{\boldsymbol{r}}, \underline{t})=-\sum_{\sigma} Z_{\sigma} \int \underline{f_{\sigma}}(\underline{\boldsymbol{r}}, \underline{\boldsymbol{v}}, \underline{t}) \mathrm{d}^{3} \underline{v},
$$

where

$$
\lambda_{\mathrm{De}}=\sqrt{\frac{T_{\mathrm{e} 0}}{4 \pi e^{2} n_{\mathrm{e} 0}}}
$$

is the electron Debye length. We assume that the Debye length is sufficiently small that we can neglect the left-hand side of (29), so quasineutrality

$$
\sum_{\sigma} Z_{\sigma} \int \underline{f_{\sigma}}(\underline{\boldsymbol{r}}, \underline{\boldsymbol{v}}, \underline{t}) \mathrm{d}^{3} \underline{v}=0
$$

holds.

In dimensionless variables the short-wavelength electrostatic potential and distribution functions satisfy

$$
\begin{gathered}
\underline{\varphi}^{\mathrm{sw}}(\underline{\boldsymbol{r}}, \underline{t}) \sim 1, \\
\underline{f_{\sigma}^{\mathrm{sw}}}(\underline{\boldsymbol{r}}, \underline{\boldsymbol{v}}, \underline{t}) \sim \epsilon_{\mathrm{s}}, \\
\hat{\boldsymbol{b}}(\underline{\boldsymbol{r}}) \cdot \nabla_{\underline{r}} \underline{\varphi}^{\mathrm{sw}}(\underline{\boldsymbol{r}}, \underline{t}) \sim 1, \\
\hat{\boldsymbol{b}}(\underline{\boldsymbol{r}}) \cdot \nabla_{\underline{\boldsymbol{r}}} \underline{f_{\sigma}^{\mathrm{sw}}}(\underline{\boldsymbol{r}}, \underline{\boldsymbol{v}}, \underline{t}) \sim \epsilon_{\mathrm{s}},
\end{gathered}
$$

$$
\begin{gathered}
\nabla_{\underline{\boldsymbol{r}}_{\perp}} \underline{\varphi}^{\mathrm{sw}}(\underline{\boldsymbol{r}}, \underline{t}) \sim 1 / \epsilon_{\mathrm{s}}, \\
\nabla_{\underline{\boldsymbol{r}}_{\perp}} \underline{f_{\sigma}^{\mathrm{sw}}}(\underline{\boldsymbol{r}}, \underline{\boldsymbol{v}}, \underline{t}) \sim 1, \\
\partial_{\underline{t}} \underline{\varphi^{\mathrm{sw}}}(\underline{\boldsymbol{r}}, \underline{t}) \sim 1, \\
\partial_{\underline{t}} f_{\sigma}^{\mathrm{sw}}(\underline{\boldsymbol{r}}, \underline{\boldsymbol{v}}, \underline{t}) \sim \epsilon_{\mathrm{s}} .
\end{gathered}
$$

The normalized functions $\varphi^{\mathrm{sw}}$ and $f_{\sigma}{ }^{\mathrm{sw}}$ are of different size due to our choice of dimensionless variables (see (22) and (23)).

As for the long-wavelength components,

$$
\begin{gathered}
\underline{\varphi}^{\mathrm{lw}}(\underline{\boldsymbol{r}}, \underline{t}) \sim 1 / \epsilon_{\mathrm{s}}, \\
\left.{\underline{f_{\sigma}^{\mathrm{lw}}}}^{\mathrm{l}} \underline{\boldsymbol{r}}, \underline{\boldsymbol{v}}, \underline{t}\right) \sim 1, \\
\nabla_{\underline{\boldsymbol{r}}} \underline{\varphi}^{\mathrm{lw}}(\underline{\boldsymbol{r}}, \underline{t}) \sim 1 / \epsilon_{\mathrm{s}}, \\
\nabla_{\underline{\boldsymbol{r}}} \underline{f_{\sigma}^{\mathrm{lw}}}(\underline{\boldsymbol{r}}, \underline{t}) \sim 1, \\
\partial_{\underline{t}} \underline{\varphi^{\mathrm{lw}}}(\underline{\boldsymbol{r}}, \underline{t}) \sim \epsilon_{\mathrm{s}}, \\
\partial_{\underline{t}} \underline{f}_{\sigma}^{\mathrm{lw}}(\underline{\boldsymbol{r}}, \underline{\boldsymbol{v}}, \underline{t}) \sim \epsilon_{\mathrm{s}}^{2} .
\end{gathered}
$$

The following notation is adopted when we expand $\underline{\varphi}^{\mathrm{lw}}(\underline{\boldsymbol{r}}, \underline{t})$ in powers of $\epsilon_{\mathrm{s}}$ :

$\underline{\varphi}^{\mathrm{lw}}(\underline{\boldsymbol{r}}, \underline{t}):=\frac{1}{\epsilon_{\mathrm{s}}} \underline{\varphi}_{0}(\underline{\boldsymbol{r}}, \underline{t})+\underline{\varphi}_{1}^{\mathrm{lw}}(\underline{\boldsymbol{r}}, \underline{t})+\epsilon_{\mathrm{s}} \underline{\varphi}^{\mathrm{lw}}(\underline{\boldsymbol{r}}, \underline{t})+O\left(\epsilon_{\mathrm{s}}^{2}\right)$.

Similarly,

$$
\underline{\varphi}^{\mathrm{sw}}(\underline{\boldsymbol{r}}, \underline{t}):=\underline{\varphi}^{\mathrm{sw}}(\underline{\boldsymbol{r}}, \underline{t})+\epsilon_{\mathrm{s}} \underline{\varphi}^{\mathrm{sw}}(\underline{\boldsymbol{r}}, \underline{t})+O\left(\epsilon_{\mathrm{s}}^{2}\right) .
$$

From now on we do not underline variables but assume that we are working with the dimensionless ones. Only in section 7 we go back to dimensionful variables.

\subsection{Gyrokinetic coordinate transformation}

The goal of the gyrokinetic expansion is to use the ordering assumptions in section 2.1 to remove from the kinetic equation the degree of freedom associated to the gyromotion, order by order. Grossly speaking, there are two typical approaches to this objective. Iterative methods act by directly averaging the equations of motion [6,39-42] to each order in the expansion parameter. Hamiltonian and Lagrangian methods rely on the machinery of analytical mechanics and the averaging procedure is implemented by requiring that certain geometrical objects be gyrophase independent [10,43-45]. Both approaches try to find new coordinates on phase space in which the slow and fast degrees of freedom are decoupled. Such coordinates, that are not uniquely defined, are called gyrokinetic coordinates.

The complete calculation of the gyrokinetic system of equations to second order is given in [10] in the phase-space Lagrangian formalism. The latter was applied to the problem of guiding-center motion by Littlejohn [46] and has been used extensively in modern formulations of gyrokinetics [45]. In [10] a change of variables is performed in (24) and (31), that 
decouples the fast degree of freedom, the gyrophase, from the slow ones in the absence of collisions. This decoupling is achieved by eliminating the dependence of the phasespace Lagrangian on the gyrophase order by order in $\epsilon_{\sigma}$. Let us denote the transformation from the new phase-space coordinates $\boldsymbol{Z} \equiv\{\boldsymbol{R}, u, \mu, \theta\}$ to the euclidean ones $\boldsymbol{X} \equiv$ $\{\boldsymbol{r}, \boldsymbol{v}\}$ by $\mathcal{T}_{\sigma}$,

$$
(\boldsymbol{r}, \boldsymbol{v})=\mathcal{T}_{\sigma}(\boldsymbol{R}, u, \mu, \theta, t) .
$$

The transformation is, in general, explicitly time-dependent and is expressed as a power series in $\epsilon_{\sigma}$. Here, $R, u, \mu$, and $\theta$ are deformations of the guiding-center position, parallel velocity, magnetic moment, and gyrophase, respectively. Namely,

$$
\begin{gathered}
\boldsymbol{R}=\boldsymbol{r}-\epsilon_{\sigma} \frac{1}{B(\boldsymbol{r})} \hat{\boldsymbol{b}}(\boldsymbol{r}) \times \boldsymbol{v}+O\left(\epsilon_{\sigma}^{2}\right), \\
u=\boldsymbol{v} \cdot \hat{\boldsymbol{b}}(\boldsymbol{r})+O\left(\epsilon_{\sigma}\right), \\
\mu=\frac{1}{2 B(\boldsymbol{r})}(\boldsymbol{v}-\boldsymbol{v} \cdot \hat{\boldsymbol{b}}(\boldsymbol{r}) \hat{\boldsymbol{b}}(\boldsymbol{r}))^{2}+O\left(\epsilon_{\sigma}\right), \\
\theta=\arctan \left(\frac{\boldsymbol{v} \cdot \hat{\boldsymbol{e}}_{2}(\boldsymbol{r})}{\boldsymbol{v} \cdot \hat{\boldsymbol{e}}_{1}(\boldsymbol{r})}\right)+O\left(\epsilon_{\sigma}\right),
\end{gathered}
$$

where the unit vectors $\hat{e}_{1}(r)$ and $\hat{e}_{2}(r)$ are orthogonal to each other and to $\hat{b}(r)$, and satisfy $\hat{e}_{1}(r) \times \hat{e}_{2}(r)=\hat{b}(r)$ at every location $r$.

We want to write the Fokker-Planck equation in gyrokinetic coordinates. Denote by $\mathcal{T}_{\sigma}^{*}$ the pull-back transformation induced by $\mathcal{T}_{\sigma}$. Acting on a function $g(X, t)$, $\mathcal{T}_{\sigma}^{*} g(\boldsymbol{Z}, t)$ is simply the function $g$ written in coordinates $\boldsymbol{Z}$, i.e.

$$
\mathcal{T}_{\sigma}^{*} g(Z, t)=g\left(\mathcal{T}_{\sigma}(Z, t), t\right)
$$

Now, defining $F_{\sigma}:=\mathcal{T}_{\sigma}^{*} f_{\sigma}$, we transform (24) and get

$$
\begin{aligned}
\partial_{t} F_{\sigma} & +\tau_{\sigma}\left\{F_{\sigma}, \bar{H}_{\sigma}\right\}_{Z} \\
& =\tau_{\sigma} \sum_{\sigma^{\prime}} \mathcal{T}_{\sigma}^{*} C_{\sigma \sigma^{\prime}}\left[\mathcal{T}_{\sigma}^{-1 *} F_{\sigma}, \mathcal{T}_{\sigma^{\prime}}^{-1 *} F_{\sigma^{\prime}}\right](Z, t),
\end{aligned}
$$

where $\mathcal{T}_{\sigma}^{-1 *}$ is the pull-back transformation that corresponds to $\mathcal{T}_{\sigma}^{-1}$, i.e. $\mathcal{T}_{\sigma}^{-1 *} F_{\sigma}(\boldsymbol{X}, t)=F_{\sigma}\left(\mathcal{T}_{\sigma}^{-1}(\boldsymbol{X}, t), t\right)$, and the Poisson bracket in the new coordinates is expressed as

$$
\begin{aligned}
\left\{G_{1}, G_{2}\right\}_{Z}=\frac{1}{\epsilon_{\sigma}}\left(\partial_{\mu} G_{1} \partial_{\theta} G_{2}-\partial_{\theta} G_{1} \partial_{\mu} G_{2}\right) \\
+\frac{1}{B_{\|, \sigma}^{*}} B_{\sigma}^{*} \cdot\left(\nabla_{R}^{*} G_{1} \partial_{u} G_{2}-\partial_{u} G_{1} \nabla_{R}^{*} G_{2}\right) \\
+\frac{\epsilon_{\sigma}}{B_{\|}^{*}} \nabla_{R}^{*} G_{1} \cdot\left(\hat{b} \times \nabla_{R}^{*} G_{2}\right),
\end{aligned}
$$

with

$$
\begin{gathered}
\boldsymbol{B}_{\sigma}^{*}(\boldsymbol{R}, u, \mu):=\boldsymbol{B}(\boldsymbol{R})+\epsilon_{\sigma} u \nabla_{\boldsymbol{R}} \times \hat{\boldsymbol{b}}(\boldsymbol{R}) \\
-\epsilon_{\sigma}^{2} \mu \nabla_{\boldsymbol{R}} \times \boldsymbol{K}(\boldsymbol{R}), \\
B_{\|, \sigma}^{*}(\boldsymbol{R}, u, \mu):=\boldsymbol{B}_{\sigma}^{*}(\boldsymbol{R}, u, \mu) \cdot \hat{\boldsymbol{b}}(\boldsymbol{R}) \\
=B(\boldsymbol{R})+\epsilon_{\sigma} u \hat{\boldsymbol{b}}(\boldsymbol{R}) \cdot \nabla_{\boldsymbol{R}} \times \hat{\boldsymbol{b}}(\boldsymbol{R}) \\
-\epsilon_{\sigma}^{2} \mu \hat{\boldsymbol{b}}(\boldsymbol{R}) \cdot \nabla_{\boldsymbol{R}} \times \boldsymbol{K}(\boldsymbol{R}),
\end{gathered}
$$

$\nabla_{R}^{*}:=\nabla_{R}-\boldsymbol{K}(\boldsymbol{R}) \partial_{\theta}$,

and

$\boldsymbol{K}(\boldsymbol{R})=\frac{1}{2} \hat{\boldsymbol{b}}(\boldsymbol{R}) \hat{\boldsymbol{b}}(\boldsymbol{R}) \cdot \nabla_{\boldsymbol{R}} \times \hat{\boldsymbol{b}}(\boldsymbol{R})-\nabla_{\boldsymbol{R}} \hat{\boldsymbol{e}}_{2}(\boldsymbol{R}) \cdot \hat{\boldsymbol{e}}_{1}(\boldsymbol{R})$.

The gyrokinetic transformation is not unique. To compare with standard references, we have chosen $(\boldsymbol{R}, u, \mu, \theta)$ so that the Poisson bracket has the form (40), precisely the same employed in [45].

In gyrokinetic variables the quasineutrality equation reads

$\sum_{\sigma} Z_{\sigma} \int\left|\operatorname{det}\left(J_{\sigma}\right)\right| F_{\sigma} \delta\left(\pi^{r}\left(\mathcal{T}_{\sigma}(Z, t)\right)-\boldsymbol{r}\right) \mathrm{d}^{6} Z=0$,

where $\pi^{r}(r, v):=r$, and the Jacobian of the transformation to $O\left(\epsilon_{\sigma}^{2}\right)$ is

$$
\left|\operatorname{det}\left(J_{\sigma}\right)\right|=B_{\|, \sigma}^{*} .
$$

We do not include in this paper all the details of the derivation of the gyrokinetic Hamiltonian and change of coordinates, and we refer the reader to $[10,14]$. Using the explicit expressions for $\bar{H}_{\sigma}$ and for $\mathcal{T}_{\sigma}^{*}$ given in those references, we will compute in section 3 the longwavelength limit of the Fokker-Planck equation (39) and the quasineutrality equation (45) up to second order in the expansion parameter $\epsilon_{\mathrm{s}}$. The manipulations are very similar to those made in [14] for the tokamak, so we will present the final equations and then turn to discuss the topics that are specific to stellarators.

Finally, we recall that the gyrokinetic equations are written naturally in terms of a function $\phi_{\sigma}$ defined as

$$
\phi_{\sigma}(\boldsymbol{R}, \mu, \theta, t):=\varphi\left(\boldsymbol{R}+\epsilon_{\sigma} \boldsymbol{\rho}(\boldsymbol{R}, \mu, \theta), t\right),
$$

with the gyroradius vector, $\rho$, given by

$\boldsymbol{\rho}(\boldsymbol{R}, \mu, \theta)=-\sqrt{\frac{2 \mu}{B(\boldsymbol{R})}}\left[\sin \theta \hat{\boldsymbol{e}}_{1}(\boldsymbol{R})-\cos \theta \hat{\boldsymbol{e}}_{2}(\boldsymbol{R})\right]$.

The gyroaverage of a function $G(\boldsymbol{R}, u, \mu, \theta)$ is defined by

$$
\langle G\rangle(\boldsymbol{R}, \mu, t):=\frac{1}{2 \pi} \int_{0}^{2 \pi} G(\boldsymbol{R}, \mu, \theta, t) \mathrm{d} \theta .
$$

It is useful to introduce the gyrophase-dependent piece of $\phi$,

$$
\tilde{\phi}_{\sigma}(\boldsymbol{R}, \mu, \theta, t):=\phi_{\sigma}(\boldsymbol{R}, \mu, \theta, t)-\left\langle\phi_{\sigma}\right\rangle(\boldsymbol{R}, \mu, t) .
$$

From the ordering and scale separation assumptions on $\varphi$, equations (32) and (33), we obtain that the short-wavelength component of $\phi_{\sigma}$ is $O(1)$, i.e.

$$
\begin{aligned}
& \phi_{\sigma}^{\mathrm{sw}}=\phi_{\sigma 1}^{\mathrm{sw}}+O\left(\epsilon_{\mathrm{s}}\right), \\
& \tilde{\phi}_{\sigma}^{\mathrm{sw}}=\tilde{\phi}_{\sigma 1}^{\mathrm{sw}}+O\left(\epsilon_{\mathrm{s}}\right) .
\end{aligned}
$$


For the long wavelength piece $\phi_{\sigma}^{\mathrm{lw}}$, we use that it is possible to expand around $\boldsymbol{r}=\boldsymbol{R}$. With the double-dot convention for an arbitrary matrix $\stackrel{\leftrightarrow}{M}$ defined by $\boldsymbol{u v}: \stackrel{\leftrightarrow}{M}=v \cdot \overleftrightarrow{M} \cdot u$, we write

$$
\begin{aligned}
& \left\langle\phi_{\sigma}^{\mathrm{lw}}\right\rangle(\boldsymbol{R}, \mu, t)=\frac{1}{\epsilon_{\mathrm{s}}} \varphi_{0}(\boldsymbol{R}, t)+\varphi_{1}^{\mathrm{lw}}(\boldsymbol{R}, t) \\
& \quad+\epsilon_{\mathrm{s}}\left(\frac{\mu}{2 \lambda_{\sigma}^{2} B(\boldsymbol{R})}(\stackrel{\boldsymbol{I}}{-}-\hat{\boldsymbol{b}}(\boldsymbol{R}) \hat{\boldsymbol{b}}(\boldsymbol{R})): \nabla_{\boldsymbol{R}} \nabla_{\boldsymbol{R}} \varphi_{0}(\boldsymbol{R}, t)\right. \\
& \left.\quad+\varphi_{2}^{\mathrm{lw}}(\boldsymbol{R}, t)\right)+O\left(\epsilon_{\mathrm{s}}^{2}\right)
\end{aligned}
$$

and

$\tilde{\phi}_{\sigma}^{\mathrm{lw}}(\boldsymbol{R}, \mu, \theta, t)=\frac{1}{\lambda_{\sigma}} \boldsymbol{\rho}(\boldsymbol{R}, \mu, \theta) \cdot \nabla_{\boldsymbol{R}} \varphi_{0}(\boldsymbol{R}, t)+O\left(\epsilon_{\mathrm{s}}\right)$,

giving $\tilde{\phi}_{\sigma}^{\text {lw }}=O(1)$. We have expanded up to first order in $\epsilon_{\mathrm{s}}$ in (52) because it will be needed later in this paper. This ordering, in which the gyroaveraged long-wavelength component is large and the short-wavelength component is small, is similar to the one used in $[47,48]$.

\section{Long-wavelength gyrokinetic equations up to second order}

As advanced in section 2.3, once the explicit expressions for the gyrokinetic transformation, $\mathcal{T}_{\sigma}^{*}$, and Hamiltonian, $\bar{H}_{\sigma}$, are known, the remaining task consists of expanding equations (39) and (45) to $O\left(\epsilon_{\mathrm{s}}^{2}\right)$ and taking the long-wavelength limit. The basic ingredients, $\mathcal{T}_{\sigma}^{*}$ and $\bar{H}_{\sigma}$ to $O\left(\epsilon_{\mathrm{s}}^{2}\right)$, have been computed for general magnetic geometry in [10]. These results were employed for the first time in [14] to obtain the long-wavelength limit of the set of gyrokinetic equations in a tokamak. Here, we perform the same calculation for stellarators. Although not emphasized or exploited in [14], a number of intermediate expressions in [14] are valid for general toroidal systems. Hence, when working out the longwavelength gyrokinetic Fokker-Planck and quasineutrality equations in the following subsections, we make use of some results of [14] and explain in detail the problems specific to stellarators. More specifically, in this section we use appendix A in [14], expand (39) and (45) to $O\left(\epsilon_{\mathrm{s}}^{2}\right)$, and take the long-wavelength limit following sections 3 and 4 of [14], but we do not assume that the magnetic field is axisymmetric as in a tokamak.

\subsection{Fokker-Planck equation at long wavelengths}

The objective in this subsection is to calculate the longwavelength limit of the gyrokinetic Fokker-Planck equation (39) up to second order in $\epsilon_{\mathrm{s}}$ for an arbitrary stellarator. We will expand $F_{\sigma}$ as

$$
F_{\sigma}=\sum_{n=0}^{\infty} \epsilon_{\sigma}^{n} F_{\sigma n}=\sum_{n=0}^{\infty} \epsilon_{\sigma}^{n} F_{\sigma n}^{\mathrm{lw}}+\sum_{n=1}^{\infty} \epsilon_{\sigma}^{n} F_{\sigma n}^{\mathrm{sw}} .
$$

From the ordering assumptions enumerated in section 2 it follows that

$$
F_{\sigma n} \sim 1, \quad n \geqslant 0,
$$

$$
\hat{\boldsymbol{b}}(\boldsymbol{R}) \cdot \nabla_{\boldsymbol{R}} F_{\sigma n} \sim 1, \quad n \geqslant 0 .
$$

The long-wavelength component of every $F_{\sigma n}$ must have perpendicular derivatives of order unity and time derivatives of order $\epsilon_{\mathrm{s}}^{2}$ in normalized variables, i.e.

$$
\begin{array}{rlrl}
\nabla_{\boldsymbol{R}_{\perp}} F_{\sigma n}^{\mathrm{lw}} \sim 1, & & n \geqslant 0, \\
\partial_{t} F_{\sigma n}^{\mathrm{lw}} \sim \epsilon_{\mathrm{s}}^{2}, & n \geqslant 0 .
\end{array}
$$

Finally, the zeroth-order distribution function must have an identically vanishing short-wavelength component. The perpendicular gradient of the rest of the short-wavelength components is of order $\epsilon_{\sigma}^{-1}$ and the time derivative is of order unity,

$$
\begin{gathered}
F_{\sigma 0}^{\mathrm{sw}} \equiv 0, \\
\nabla_{\boldsymbol{R}_{\perp} F_{\sigma n}^{\mathrm{sw}}} \sim \epsilon_{\sigma}^{-1}, \quad n \geqslant 1, \\
\partial_{t} F_{\sigma n}^{\mathrm{sw}} \sim 1, \quad n \geqslant 1 .
\end{gathered}
$$

3.1.1. Long-wavelength Fokker-Planck equation to $O\left(\epsilon_{\sigma}^{-1}\right)$. The coefficient of $\epsilon_{\sigma}^{-1}$ in (39) simply gives

$$
-\tau_{\sigma} B \partial_{\theta} F_{\sigma 0}=0
$$

implying that $F_{\sigma 0}$ is independent of $\theta$.

3.1.2. Long-wavelength Fokker-Planck equation to $O\left(\epsilon_{\sigma}^{0}\right)$. From the $O\left(\epsilon_{\sigma}^{0}\right)$ terms in (39) it is deduced, after a calculation identical to the one in [14], that the first-order distribution function is gyrophase independent,

$$
\partial_{\theta} F_{\sigma 1}=0
$$

that $F_{\sigma 0}$ is a Maxwellian with zero mean flow,

$F_{\sigma 0}(\boldsymbol{R}, u, \mu, t)=\frac{n_{\sigma}}{\left(2 \pi T_{\sigma}\right)^{3 / 2}} \exp \left(-\frac{\mu B(\boldsymbol{R})+u^{2} / 2}{T_{\sigma}}\right)$,

and that $n_{\sigma} \equiv n_{\sigma}(\psi, t), T_{\sigma} \equiv T_{\sigma}(\psi, t)$, and $\varphi_{0} \equiv \varphi_{0}(\psi, t)$ are flux functions. The temperature has to be the same for all the species; that is, $T_{\sigma}=T_{\sigma^{\prime}}$ for every pair $\sigma, \sigma^{\prime}$. If a subsidiary expansion in the mass ratio $\sqrt{m_{\mathrm{e}} / m_{\mathrm{i}}} \ll 1$ is performed, or equivalently, if $\tau_{\mathrm{e}} \sim \lambda_{\mathrm{e}} \gg 1$ is used, the electron temperature can decouple from the ion temperature.

3.1.3. Long-wavelength Fokker-Planck equation to $O\left(\epsilon_{\sigma}\right)$. The equations presented in this subsection involve the collision operator, which is typically written in coordinates $\boldsymbol{X} \equiv$ $(\boldsymbol{r}, \boldsymbol{v})$. We avoid transforming the kernel that defines this operator by transforming, instead, the gyrokinetic distribution function $F_{\sigma}(\boldsymbol{R}, u, \mu, \theta, t)$ from gyrokinetic coordinates $\boldsymbol{Z} \equiv$ $(\boldsymbol{R}, u, \mu, \theta)$ to euclidean coordinates $\boldsymbol{X} \equiv(\boldsymbol{r}, \boldsymbol{v})$. We denote the coefficients of the expansion of $\mathcal{T}_{\sigma}$ and its inverse $\mathcal{T}_{\sigma}^{-1}$ by

$$
\begin{aligned}
\boldsymbol{X}= & \mathcal{T}_{\sigma}(\boldsymbol{Z}, t)=\mathcal{T}_{\sigma, 0}(\boldsymbol{Z}, t)+\epsilon_{\sigma} \mathcal{T}_{\sigma, 1}(\boldsymbol{Z}, t)+O\left(\epsilon_{\sigma}^{2}\right), \\
\boldsymbol{Z}= & \mathcal{T}_{\sigma}^{-1}(\boldsymbol{X}, t)=\mathcal{T}_{\sigma, 0}^{-1}(\boldsymbol{X}, t)+\epsilon_{\sigma} \mathcal{T}_{\sigma, 1}^{-1}(\boldsymbol{X}, t) \\
& +\epsilon_{\sigma}^{2} \mathcal{T}_{\sigma, 2}^{-1}(\boldsymbol{X}, t)+O\left(\epsilon_{\sigma}^{3}\right) .
\end{aligned}
$$


In the present section we only need $\mathcal{T}_{\sigma, 0}$, the transformation $\mathcal{T}_{\sigma}$ for $\epsilon_{\sigma}=0$ (compare with (37)),

$\mathcal{T}_{\sigma, 0}(\boldsymbol{R}, u, \mu, \theta)=(\boldsymbol{R}, u \hat{\boldsymbol{b}}(\boldsymbol{R})+\boldsymbol{\rho}(\boldsymbol{R}, \mu, \theta) \times \boldsymbol{B}(\boldsymbol{R}))$.

In subsequent subsections some pieces of $\mathcal{T}_{\sigma, 1}$ and $\mathcal{T}_{\sigma, 2}$ are required.

From the Fokker-Planck equation to $O\left(\epsilon_{\sigma}\right)$ one gets an equation for the gyrophase-dependent piece of $F_{\sigma 2}^{1 \mathrm{w}}$,

$-B \partial_{\theta}\left(F_{\sigma 2}^{\mathrm{lw}}-\left\langle F_{\sigma 2}^{\mathrm{lw}}\right\rangle\right)$

$$
\begin{aligned}
& =\sum_{\sigma^{\prime}} \mathcal{T}_{\sigma, 0}^{*} C_{\sigma \sigma^{\prime}}\left[\frac { 1 } { T _ { \sigma } } \left(\boldsymbol{v} \cdot \boldsymbol{V}_{\sigma}^{\mathrm{p}}\right.\right. \\
& \left.\left.+\left(\frac{v^{2}}{2 T_{\sigma}}-\frac{5}{2}\right) \boldsymbol{v} \cdot \boldsymbol{V}_{\sigma}^{\mathrm{T}}\right) \mathcal{T}_{\sigma, 0}^{-1 *} F_{\sigma 0}, \mathcal{T}_{\sigma^{\prime}, 0}^{-1 *} F_{\sigma^{\prime} 0}\right] \\
& +\sum_{\sigma^{\prime}} \frac{\lambda_{\sigma}}{\lambda_{\sigma^{\prime}}} \mathcal{T}_{\sigma, 0}^{*} C_{\sigma \sigma^{\prime}}\left[\mathcal{T}_{\sigma, 0}^{-1 *} F_{\sigma 0}, \frac{1}{T_{\sigma^{\prime}}}\left(\boldsymbol{v} \cdot \boldsymbol{V}_{\sigma^{\prime}}^{\mathrm{p}}\right.\right. \\
& \left.\left.+\left(\frac{v^{2}}{2 T_{\sigma^{\prime}}}-\frac{5}{2}\right) \boldsymbol{v} \cdot \boldsymbol{V}_{\sigma^{\prime}}^{\mathrm{T}}\right) \mathcal{T}_{\sigma^{\prime}, 0}^{-1 *} F_{\sigma^{\prime} 0}\right],
\end{aligned}
$$

where the velocities $\boldsymbol{V}_{\sigma}^{\mathrm{p}}$ and $\boldsymbol{V}_{\sigma}^{\mathrm{T}}$ are defined by

$$
\boldsymbol{V}_{\sigma}^{\mathrm{p}}:=\frac{1}{n_{\sigma} B} \hat{\boldsymbol{b}} \times \nabla p_{\sigma}, \quad \boldsymbol{V}_{\sigma}^{\mathrm{T}}:=\frac{1}{B} \hat{\boldsymbol{b}} \times \nabla T_{\sigma},
$$

and $p_{\sigma}:=n_{\sigma} T_{\sigma}$ is the pressure of species $\sigma$.

One also gets an equation for $F_{\sigma 1}^{\mathrm{lw}}$ (recall from section 3.1.2 that $F_{\sigma 1}$ is gyrophase-independent),

$$
\begin{aligned}
(u \hat{\boldsymbol{b}} & \left.\cdot \nabla_{\boldsymbol{R}}-\mu \hat{\boldsymbol{b}} \cdot \nabla_{\boldsymbol{R}} B \partial_{u}\right) F_{\sigma 1}^{\mathrm{lw}} \\
& +\left(\frac{Z_{\sigma}}{T_{\sigma}} \partial_{\psi} \varphi_{0}+\Upsilon_{\sigma}\right) \boldsymbol{v}_{M} \cdot \nabla_{\boldsymbol{R}} \psi F_{\sigma 0} \\
& +\frac{Z_{\sigma} \lambda_{\sigma}}{T_{\sigma}} u \hat{\boldsymbol{b}} \cdot \nabla_{\boldsymbol{R}} \varphi_{1}^{\mathrm{lw}} F_{\sigma 0} \\
= & \sum_{\sigma^{\prime}} \mathcal{T}_{\sigma, 0}^{*} C_{\sigma \sigma^{\prime}}\left[\mathcal{T}_{\sigma, 0}^{-1 *} F_{\sigma 1}^{\mathrm{lw}}, \mathcal{T}_{\sigma^{\prime}, 0}^{-1 *} F_{\sigma^{\prime} 0}\right] \\
& +\sum_{\sigma^{\prime}} \frac{\lambda_{\sigma}}{\lambda_{\sigma^{\prime}}} \mathcal{T}_{\sigma, 0}^{*} C_{\sigma \sigma^{\prime}}\left[\mathcal{T}_{\sigma, 0}^{-1 *} F_{\sigma 0}, \mathcal{T}_{\sigma^{\prime}, 0}^{-1 *} F_{\sigma^{\prime} 1}^{\mathrm{lw}}\right] .
\end{aligned}
$$

This is the well-known drift-kinetic equation for a general stellarator. Here,

$$
\Upsilon_{\sigma}:=\partial_{\psi} \ln n_{\sigma}+\left(\frac{u^{2} / 2+\mu B}{T_{\sigma}}-\frac{3}{2}\right) \partial_{\psi} \ln T_{\sigma}
$$

and

$$
\boldsymbol{v}_{M}:=\boldsymbol{v}_{\kappa}+\boldsymbol{v}_{\nabla B}
$$

is the magnetic drift velocity, where

$$
\begin{aligned}
\boldsymbol{v}_{\kappa} & :=\frac{u^{2}}{B} \hat{\boldsymbol{b}} \times \kappa, \\
\boldsymbol{v}_{\nabla B} & :=\frac{\mu}{B} \hat{\boldsymbol{b}} \times \nabla_{R} B,
\end{aligned}
$$

and

$$
\kappa:=\hat{b} \cdot \nabla_{R} \hat{b}
$$

is the magnetic field curvature.
Finally, in terms of the non-adiabatic part of the distribution function,

$$
G_{\sigma 1}^{\mathrm{lw}}:=F_{\sigma 1}^{\mathrm{lw}}+\frac{Z_{\sigma} \lambda_{\sigma}}{T_{\sigma}} \varphi_{1}^{\mathrm{lw}} F_{\sigma 0},
$$

the first-order Fokker-Planck equation reads

$$
\begin{aligned}
& \left(u \hat{\boldsymbol{b}} \cdot \nabla_{\boldsymbol{R}}-\mu \hat{\boldsymbol{b}} \cdot \nabla_{\boldsymbol{R}} B \partial_{u}\right) G_{\sigma 1}^{\mathrm{lw}} \\
& \quad+\left(\frac{Z_{\sigma}}{T_{\sigma}} \partial_{\psi} \varphi_{0}+\Upsilon_{\sigma}\right) \boldsymbol{v}_{M} \cdot \nabla_{\boldsymbol{R}} \psi F_{\sigma 0} \\
& =\sum_{\sigma^{\prime}} \mathcal{T}_{\sigma, 0}^{*} C_{\sigma \sigma^{\prime}}\left[\mathcal{T}_{\sigma, 0}^{-1 *} G_{\sigma 1}^{\mathrm{lw}}, \mathcal{T}_{\sigma^{\prime}, 0}^{-1 *} F_{\sigma^{\prime} 0}\right] \\
& \quad+\sum_{\sigma^{\prime}} \frac{\lambda_{\sigma}}{\lambda_{\sigma^{\prime}}} \mathcal{T}_{\sigma, 0}^{*} C_{\sigma \sigma^{\prime}}\left[\mathcal{T}_{\sigma, 0}^{-1 *} F_{\sigma 0}, \mathcal{T}_{\sigma^{\prime}, 0}^{-1 *} G_{\sigma^{\prime} 1}^{\mathrm{lw}}\right] .
\end{aligned}
$$

The electrostatic potential $\varphi_{1}^{\mathrm{lw}}$ does not appear in the collision operator due to property (A.10).

3.1.4. Short-wavelength Fokker-Planck and quasineutrality equations to $O\left(\epsilon_{\sigma}\right)$. We will learn in section 3.1.5 that the long-wavelength Fokker-Planck equation to $O\left(\epsilon_{\mathrm{s}}^{2}\right)$ involves terms containing $F_{\sigma 1}^{\mathrm{sw}}$ and $\phi_{\sigma 1}^{\mathrm{sw}}$. Next, we give the equations determining these pieces. In order to write in a compact and precise way the collision terms of the short-wavelength equations, it is convenient to define a new operator $\mathbb{T}_{\sigma, 0}$ acting on phase-space functions $F(\boldsymbol{R}, u, \mu, \theta)$. Namely,

$$
\begin{aligned}
& \mathbb{T}_{\sigma, 0} F(\boldsymbol{r}, \boldsymbol{v}):=F\left(\boldsymbol{r}-\epsilon_{\sigma} \frac{1}{B(\boldsymbol{r})} \hat{\boldsymbol{b}}(\boldsymbol{r}) \times \boldsymbol{v}, \boldsymbol{v} \cdot \hat{\boldsymbol{b}}(\boldsymbol{r}), \frac{v_{\perp}^{2}}{2 B(\boldsymbol{r})},\right. \\
& \left.\quad \arctan \left(\frac{\boldsymbol{v} \cdot \hat{\boldsymbol{e}}_{2}(\boldsymbol{r})}{\boldsymbol{v} \cdot \hat{\boldsymbol{e}}_{1}(\boldsymbol{r})}\right)\right) .
\end{aligned}
$$

This operator is useful to write some expressions that contain the short-wavelength pieces of the distribution function and the potential, for which it is not possible to expand the dependence on $\boldsymbol{r}-\epsilon_{\sigma} B(\boldsymbol{r})^{-1} \hat{\boldsymbol{b}}(\boldsymbol{r}) \times \boldsymbol{v}$ around $\boldsymbol{r}$.

The short-wavelength, $O\left(\epsilon_{\mathrm{s}}\right)$ terms of (39) yield

$$
\begin{aligned}
\frac{1}{\tau_{\sigma}} \partial_{t} & F_{\sigma 1}^{\mathrm{sw}}+\left(u \hat{\boldsymbol{b}} \cdot \nabla_{\boldsymbol{R}}-\mu \hat{\boldsymbol{b}} \cdot \nabla_{\boldsymbol{R}} B \partial_{u}\right) F_{\sigma 1}^{\mathrm{sw}} \\
+ & {\left[\frac{Z_{\sigma} \lambda_{\sigma}}{B}\left(\hat{\boldsymbol{b}} \times \nabla_{\boldsymbol{R}_{\perp} / \epsilon_{\sigma}}\left\langle\phi_{\sigma 1}^{\mathrm{sw}}\right\rangle\right) \cdot \nabla_{\boldsymbol{R}_{\perp} / \epsilon_{\sigma}} F_{\sigma 1}^{\mathrm{sw}}\right]^{\mathrm{sw}} } \\
+ & \left(\boldsymbol{v}_{M}+\boldsymbol{v}_{E, \sigma}^{(0)}\right) \cdot \nabla_{\boldsymbol{R}_{\perp} / \epsilon_{\sigma}} F_{\sigma 1}^{\mathrm{sw}} \\
+ & \frac{Z_{\sigma} \lambda_{\sigma}}{B}\left(\hat{\boldsymbol{b}} \times \nabla_{\boldsymbol{R}_{\perp} / \epsilon_{\sigma}}\left\langle\phi_{\sigma 1}^{\mathrm{sw}}\right\rangle\right) \cdot \nabla_{\boldsymbol{R}} F_{\sigma 0} \\
& -Z_{\sigma} \lambda_{\sigma}\left(\hat{\boldsymbol{b}} \cdot \nabla_{\boldsymbol{R}}\left\langle\phi_{\sigma 1}^{\mathrm{sw}}\right\rangle\right. \\
& \left.+\frac{u}{B}(\hat{\boldsymbol{b}} \times \boldsymbol{\kappa}) \cdot \nabla_{\boldsymbol{R}_{\perp} / \epsilon_{\sigma}}\left\langle\phi_{\sigma 1}^{\mathrm{sw}}\right\rangle\right) \partial_{u} F_{\sigma 0} \\
= & \sum_{\sigma^{\prime}}\left\langle\mathcal { T } _ { \mathrm { NP } , \sigma } ^ { * } C _ { \sigma \sigma ^ { \prime } } \left[\mathbb{T}_{\sigma, 0} F_{\sigma 1}^{\mathrm{sw}}\right.\right. \\
& \left.\left.-\frac{Z_{\sigma} \lambda_{\sigma}}{T_{\sigma}} \mathbb{T}_{\sigma, 0} \tilde{\phi}_{\sigma 1}^{\mathrm{sw}} \mathcal{T}_{\sigma, 0}^{-1 *} F_{\sigma 0}, \mathcal{T}_{\sigma^{\prime}, 0}^{-1 *} F_{\sigma^{\prime} 0}\right]\right\rangle
\end{aligned}
$$




$$
\begin{aligned}
& +\sum_{\sigma^{\prime}} \frac{\lambda_{\sigma}}{\lambda_{\sigma^{\prime}}}\left\langle\mathcal { T } _ { \mathrm { NP } , \sigma } ^ { * } C _ { \sigma \sigma ^ { \prime } } \left[\mathcal{T}_{\sigma, 0}^{-1 *} F_{\sigma 0}, \mathbb{T}_{\sigma^{\prime}, 0} F_{\sigma^{\prime} 1}^{\mathrm{sw}}\right.\right. \\
& \left.\left.-\frac{Z_{\sigma^{\prime}} \lambda_{\sigma^{\prime}}}{T_{\sigma^{\prime}}} \mathbb{T}_{\sigma^{\prime}, 0} \tilde{\phi}_{\sigma^{\prime} 1}^{\mathrm{sw}} \mathcal{T}_{\sigma^{\prime}, 0}^{-1 *} F_{\sigma^{\prime} 0}\right]\right\rangle,
\end{aligned}
$$

where

$$
\boldsymbol{v}_{E, \sigma}^{(0)}=\frac{Z_{\sigma}}{B} \hat{\boldsymbol{b}} \times \nabla_{R} \varphi_{0}
$$

and the transformation $(\boldsymbol{r}, \boldsymbol{v})=\mathcal{T}_{\mathrm{NP}, \sigma}(\boldsymbol{R}, u, \mu, \theta)$ is defined by

$$
\begin{gathered}
\boldsymbol{r}=\boldsymbol{R}+\epsilon_{\sigma} \boldsymbol{\rho}(\boldsymbol{R}, \mu, \theta), \\
\boldsymbol{v}=u \hat{\boldsymbol{b}}(\boldsymbol{R})+\boldsymbol{\rho}(\boldsymbol{R}, \mu, \theta) \times \boldsymbol{B}(\boldsymbol{R}) .
\end{gathered}
$$

This coincides with the non-perturbative transformation introduced in [10] and that is why we have kept the notation $\mathcal{T}_{\mathrm{NP}, \sigma}$.

The short-wavelength, $O\left(\epsilon_{\mathrm{s}}\right)$ terms of (45) give

$$
\begin{aligned}
\sum_{\sigma} \frac{Z_{\sigma}}{\lambda_{\sigma}} & \int B\left[-Z_{\sigma} \lambda_{\sigma} \widetilde{\phi}_{\sigma 1}^{\mathrm{sw}}\left(\boldsymbol{r}-\epsilon_{\sigma} \rho(\boldsymbol{r}, \mu, \theta), \mu, \theta, t\right)\right. \\
& \times \frac{F_{\sigma 0}(\boldsymbol{r}, u, \mu, t)}{T_{\sigma}(\boldsymbol{r}, t)} \\
& \left.+F_{\sigma 1}^{\mathrm{sw}}\left(\boldsymbol{r}-\epsilon_{\sigma} \boldsymbol{\rho}(\boldsymbol{r}, \mu, \theta), u, \mu, t\right)\right] \mathrm{d} u \mathrm{~d} \mu \mathrm{d} \theta=0 .
\end{aligned}
$$

Equations (75) and (78) constitute what is usually understood by 'the $\delta f$ gyrokinetic set of equations' for electrostatic turbulence.

3.1.5. Long-wavelength Fokker-Planck equation to $O\left(\epsilon_{\sigma}^{2}\right)$. The gyroaverage of the pieces of order $\epsilon_{\sigma}^{2}$ in (39) yields

$$
\begin{aligned}
(u \hat{\boldsymbol{b}} & \left.\cdot \nabla_{\boldsymbol{R}}-\mu \hat{\boldsymbol{b}} \cdot \nabla_{\boldsymbol{R}} B \partial_{u}\right)\left\langle F_{\sigma 2}^{\mathrm{lw}}\right\rangle+\frac{\lambda_{\sigma}^{2}}{\tau_{\sigma}} \partial_{\epsilon_{\mathrm{s}}^{2} t} F_{\sigma 0} \\
& +\left(\boldsymbol{v}_{M}+\boldsymbol{v}_{E, \sigma}^{(0)}\right) \cdot \nabla_{\boldsymbol{R}} F_{\sigma 1}^{\mathrm{lw}} \\
& +\left[-Z_{\sigma} \lambda_{\sigma} \hat{\boldsymbol{b}} \cdot \nabla_{\boldsymbol{R}} \varphi_{1}^{\mathrm{lw}}+u \boldsymbol{\kappa} \cdot\left(\boldsymbol{v}_{\nabla B}+\boldsymbol{v}_{E, \sigma}^{(0)}\right)\right] \partial_{u} F_{\sigma 1}^{\mathrm{lw}} \\
& +\left[\boldsymbol{v}_{E, \sigma}^{(1)}-\frac{u}{B}\left(\hat{\boldsymbol{b}} \cdot \nabla_{\boldsymbol{R}} \times \hat{\boldsymbol{b}}\right)\left(\boldsymbol{v}_{M}+\boldsymbol{v}_{E, \sigma}^{(0)}\right)\right. \\
& \left.-\frac{u \mu}{B}\left(\nabla_{\boldsymbol{R}} \times \boldsymbol{K}\right)_{\perp}+Z_{\sigma} \lambda_{\sigma} \partial_{u} \Psi_{\phi B, \sigma}^{\mathrm{lw}} \hat{\boldsymbol{b}}+\partial_{u} \Psi_{B, \sigma} \hat{\boldsymbol{b}}\right] \cdot \nabla_{\boldsymbol{R}} F_{\sigma 0} \\
& -\left\{Z_{\sigma} \lambda_{\sigma}^{2} \hat{\boldsymbol{b}} \cdot \nabla_{\boldsymbol{R}}\left[\varphi_{2}^{\mathrm{lw}}+\frac{\mu}{2 \lambda_{\sigma}^{2} B}(\stackrel{\boldsymbol{I}}{B} \hat{\boldsymbol{b}} \hat{\boldsymbol{b}}): \nabla_{\boldsymbol{R}} \nabla_{\boldsymbol{R}} \varphi_{0}\right]\right. \\
& +\hat{\boldsymbol{b}} \cdot \nabla_{\boldsymbol{R}} \Psi_{B, \sigma}+Z_{\sigma} \lambda_{\sigma} \hat{\boldsymbol{b}} \cdot \nabla_{\boldsymbol{R}} \Psi_{\phi B, \sigma}^{1 \mathrm{w}}+Z_{\sigma}^{2} \lambda_{\sigma}^{2} \hat{\boldsymbol{b}} \cdot \nabla_{\boldsymbol{R}} \Psi_{\phi, \sigma}^{\mathrm{lw}} \\
& -u \boldsymbol{\kappa} \cdot \boldsymbol{v}_{E, \sigma}^{(1)}+\left[\frac{u^{2}}{B}\left(\hat{\boldsymbol{b}} \cdot \nabla_{\boldsymbol{R}} \times \hat{\boldsymbol{b}}\right) \boldsymbol{\kappa}\right. \\
+ & \left.\left.\mu\left(\left(\nabla_{\boldsymbol{R}} \times \boldsymbol{K}\right) \times \hat{\boldsymbol{b}}\right)\right] \cdot\left(\boldsymbol{v}_{\nabla B}+\boldsymbol{v}_{E, \sigma}^{(0)}\right)\right\} \partial_{u} F_{\sigma 0}
\end{aligned}
$$

$$
\begin{aligned}
& +\frac{Z_{\sigma} \lambda_{\sigma}}{B}\left[\nabla_{\boldsymbol{R}} \cdot\left(\hat{\boldsymbol{b}} \times \nabla_{\boldsymbol{R}_{\perp} / \epsilon_{\sigma}}\left\langle\phi_{\sigma 1}^{\mathrm{sw}}\right\rangle F_{\sigma 1}^{\mathrm{sw}}\right)\right]^{\mathrm{lw}} \\
& -Z_{\sigma} \lambda_{\sigma} \partial_{u}\left[\left(\hat{\boldsymbol{b}} \cdot \nabla_{\boldsymbol{R}}\left\langle\phi_{\sigma 1}^{\mathrm{sw}}\right\rangle\right.\right. \\
& \left.\left.+\frac{u}{B}(\hat{\boldsymbol{b}} \times \boldsymbol{\kappa}) \cdot \nabla_{\boldsymbol{R}_{\perp} / \epsilon_{\sigma}}\left\langle\phi_{\sigma 1}^{\mathrm{sw}}\right\rangle\right) F_{\sigma 1}^{\mathrm{sw}}\right]^{\mathrm{lw}} \\
& =\sum_{\sigma^{\prime}}\left\langle\left[\mathcal{T}_{\sigma, 1}^{*} C_{\sigma \sigma^{\prime}}^{(1)}\right]^{\mathrm{lw}}\right\rangle+\sum_{\sigma^{\prime}}\left\langle\mathcal{T}_{\sigma, 0}^{*} C_{\sigma \sigma^{\prime}}^{(2) \mathrm{lw}}\right\rangle .
\end{aligned}
$$

Here,

$$
\begin{aligned}
& \boldsymbol{v}_{E, \sigma}^{(1)}=\frac{Z_{\sigma} \lambda_{\sigma}}{B} \hat{\boldsymbol{b}} \times \nabla_{\boldsymbol{R}} \varphi_{1}^{\mathrm{lw}} \\
& \Psi_{\phi, \sigma}^{\mathrm{lw}}=-\frac{1}{2 \lambda_{\sigma}^{2} B^{2}}\left|\nabla_{\boldsymbol{R}} \varphi_{0}\right|^{2}-\frac{1}{2 B} \partial_{\mu}\left[\left\langle\left(\widetilde{\phi}_{\sigma 1}^{\mathrm{sw}}\right)^{2}\right\rangle\right]^{1 \mathrm{w}} \\
& \Psi_{\phi B, \sigma}^{\mathrm{lw}}=-\frac{3 \mu}{2 \lambda_{\sigma} B^{2}} \nabla_{\boldsymbol{R}} B \cdot \nabla_{\boldsymbol{R}} \varphi_{0}-\frac{u^{2}}{\lambda_{\sigma} B^{2}}\left(\hat{\boldsymbol{b}} \cdot \nabla_{\boldsymbol{R}} \hat{\boldsymbol{b}}\right) \cdot \nabla_{\boldsymbol{R}} \varphi_{0}
\end{aligned}
$$

and

$$
\begin{aligned}
\Psi_{B, \sigma}= & -\frac{3 u^{2} \mu}{2 B^{2}} \hat{\boldsymbol{b}} \cdot \nabla_{\boldsymbol{R}} \hat{\boldsymbol{b}} \cdot \nabla_{\boldsymbol{R}} B \\
& +\frac{\mu^{2}}{4 B}(\stackrel{\leftrightarrow}{\boldsymbol{I}}-\hat{\boldsymbol{b}} \hat{\boldsymbol{b}}): \nabla_{\boldsymbol{R}} \nabla_{\boldsymbol{R}} \boldsymbol{B} \cdot \hat{\boldsymbol{b}} \\
& -\frac{3 \mu^{2}}{4 B^{2}}\left|\nabla_{\boldsymbol{R}_{\perp}} B\right|^{2}+\frac{u^{2} \mu}{2 B} \nabla_{\boldsymbol{R}} \hat{\boldsymbol{b}}: \nabla_{\boldsymbol{R}} \hat{\boldsymbol{b}} \\
& +\left(\frac{\mu^{2}}{8}-\frac{u^{2} \mu}{4 B}\right) \nabla_{\boldsymbol{R}} \hat{\boldsymbol{b}}:\left(\nabla_{\boldsymbol{R}} \hat{\boldsymbol{b}}\right)^{\mathrm{T}} \\
& -\left(\frac{3 u^{2} \mu}{8 B}+\frac{\mu^{2}}{16}\right)\left(\nabla_{\boldsymbol{R}} \cdot \hat{\boldsymbol{b}}\right)^{2} \\
& +\left(\frac{3 u^{2} \mu}{2 B}-\frac{u^{4}}{2 B^{2}}\right)\left|\hat{\boldsymbol{b}} \cdot \nabla_{\boldsymbol{R}} \hat{\boldsymbol{b}}\right|^{2} \\
& +\left(\frac{u^{2} \mu}{8 B}-\frac{\mu^{2}}{16}\right)\left(\hat{\boldsymbol{b}} \cdot \nabla_{\boldsymbol{R}} \times \hat{\boldsymbol{b}}\right)^{2} .
\end{aligned}
$$

The collisional terms on the right side of (79) are spelled out in appendix B.

The fact that the time derivative of $F_{\sigma 0}$ appears in (79), and not in lower-order pieces of the equations, is important. It means that in this theoretical framework transport equations for density and energy are obtained from the $O\left(\epsilon_{\mathrm{s}}^{2}\right)$ pieces of the equation. Specifically, we show in section 5 that such transport equations emerge as solvability conditions of (79).

Equation (79) is recast in appendix D into a form, (D.9), that is especially well suited to work out the density and energy transport equations in section 5 .

\subsection{Long-wavelength quasineutrality equation}

Since the calculation of the quasineutrality equation (45) at long wavelengths is identical for tokamaks and stellarators, we simply state the result derived in [14] for tokamaks. 
To order $\epsilon_{\mathrm{s}}^{0}$,

$$
\sum_{\sigma} Z_{\sigma} n_{\sigma}(\boldsymbol{r}, t)=0
$$

To order $\epsilon_{\mathrm{s}}$,

$$
\sum_{\sigma} \frac{Z_{\sigma}}{\lambda_{\sigma}} \int B(\boldsymbol{r}) F_{\sigma 1}^{\mathrm{lw}}(\boldsymbol{r}, u, \mu, t) \mathrm{d} u \mathrm{~d} \mu \mathrm{d} \theta=0 .
$$

To order $\epsilon_{\mathrm{s}}^{2}$

$$
\begin{aligned}
\sum_{\sigma} \frac{Z_{\sigma}}{\lambda_{\sigma}^{2}}\left[\int\left(B F_{\sigma 2}^{1 \mathrm{w}}+u \hat{\boldsymbol{b}} \cdot\left(\nabla_{r} \times \hat{\boldsymbol{b}}\right) F_{\sigma 1}^{\mathrm{lw}}\right) \mathrm{d} u \mathrm{~d} \mu \mathrm{d} \theta\right. \\
-\hat{\boldsymbol{b}} \cdot\left(\nabla_{r} \times \boldsymbol{K}\right) \frac{n_{\sigma} T_{\sigma}}{B^{2}}+\nabla_{r} \cdot\left(\frac{3}{2} \frac{\nabla_{\boldsymbol{r}_{\perp}} B}{B^{3}} n_{\sigma} T_{\sigma}\right) \\
+\frac{1}{2} \nabla_{r} \nabla_{r}:\left((\overleftrightarrow{\boldsymbol{I}}-\hat{\boldsymbol{b}} \hat{\boldsymbol{b}}) \frac{n_{\sigma} T_{\sigma}}{B^{2}}\right) \\
\left.+\nabla_{\boldsymbol{r}} \cdot\left(\frac{n_{\sigma} T_{\sigma}}{B^{2}} \kappa+\frac{Z_{\sigma} n_{\sigma}}{B^{2}} \nabla_{r} \varphi_{0}\right)\right]=0 .
\end{aligned}
$$

Here, everything is evaluated at $\boldsymbol{R}=\boldsymbol{r}$. In writing the arguments of some functions we have stressed that they are evaluated at $\boldsymbol{R}=\boldsymbol{r}$, e.g. $n_{\sigma}(\boldsymbol{r})$, but we should not forget that $n_{\sigma}$, for example, depends only on $\psi$ in flux coordinates.

\section{The long-wavelength radial electric field cannot be directly determined from the quasineutrality equation}

At this point we have already derived the Fokker-Planck and quasineutrality equations to $O\left(\epsilon_{\mathrm{s}}^{2}\right)$. One might think that the quasineutrality equation is enough to solve for the longwavelength radial electric field, but this is not true for any stellarator. The argument is the same as that given in [14] for the tokamak, but it is useful to restate it briefly here.

Define

$h_{\sigma j}=\left[\frac{n_{\sigma j}}{n_{\sigma}}+\left(\frac{\mu B+u^{2} / 2}{T_{\sigma}}-\frac{3}{2}\right) \frac{T_{\sigma j}}{T_{\sigma}}\right] F_{\sigma 0}, \quad j=1,2$,

for an arbitrary set of flux functions $\left\{n_{\sigma j}(\psi, t), T_{\sigma j}(\psi, t)\right\}_{\sigma}$ with the only restriction $T_{\sigma j} / \lambda_{\sigma}^{j}=T_{\sigma^{\prime} j} / \lambda_{\sigma^{\prime}}^{j}$, for all $\sigma, \sigma^{\prime}$. If $F_{\sigma 1}^{\mathrm{lw}}$ and $\left\langle F_{\sigma 2}^{\mathrm{lw}}\right\rangle$ are solutions of the first and second-order Fokker-Planck equations, (66) (equivalently, (73)) and (79), then so are $F_{\sigma 1}^{\mathrm{lw}}+h_{\sigma 1}$ and $\left\langle F_{\sigma 2}^{\mathrm{lw}}\right\rangle+h_{\sigma 2}$. That is, (87) gives the kernel of the operator acting on $F_{\sigma 1}^{\mathrm{lw}}$ in (66) and on $\left\langle F_{\sigma 2}^{\mathrm{lw}}\right\rangle$ in (79). The freedom due to the existence of a non-zero kernel can be removed by imposing conditions such as

$\left\langle\int B F_{\sigma 1}^{\mathrm{lw}} \mathrm{d} u \mathrm{~d} \mu \mathrm{d} \theta\right\rangle_{\psi}=0$ for every $\sigma$,

$\left\langle\int B\left\langle F_{\sigma 2}^{\mathrm{lw}}\right\rangle \mathrm{d} u \mathrm{~d} \mu \mathrm{d} \theta\right\rangle_{\psi}=0$ for every $\sigma$,

$\left\langle\sum_{\sigma} \frac{1}{\lambda_{\sigma}} \int B\left(u^{2} / 2+\mu B\right) F_{\sigma 1}^{\mathrm{lw}} \mathrm{d} u \mathrm{~d} \mu \mathrm{d} \theta\right\rangle_{\psi}=0$ and

$\left\langle\sum_{\sigma} \frac{1}{\lambda_{\sigma}^{2}} \int B\left(u^{2} / 2+\mu B\right)\left\langle F_{\sigma 2}^{\mathrm{lw}}\right\rangle \mathrm{d} u \mathrm{~d} \mu \mathrm{d} \theta\right\rangle_{\psi}=0$.

Here, we have used the definition of the flux-surface average of a function $G(\psi, \Theta, \zeta)$, given by [49]

$$
\langle G\rangle_{\psi}:=\frac{\int_{0}^{2 \pi} \int_{0}^{2 \pi} \sqrt{g} G(\psi, \Theta, \zeta) \mathrm{d} \Theta \mathrm{d} \zeta}{\int_{0}^{2 \pi} \int_{0}^{2 \pi} \sqrt{g} \mathrm{~d} \Theta \mathrm{d} \zeta},
$$

where

$$
\sqrt{g}:=\frac{1}{\nabla_{R} \psi \cdot\left(\nabla_{R} \Theta \times \nabla_{R} \zeta\right)}
$$

is the square root of the determinant of the metric tensor in coordinates $\{\psi, \Theta, \zeta\}$. It will also be useful to define the volume enclosed by the flux surface labeled by $\psi$,

$$
V(\psi)=\int_{0}^{\psi} \int_{0}^{2 \pi} \int_{0}^{2 \pi} \sqrt{g} \mathrm{~d} \psi^{\prime} \mathrm{d} \Theta \mathrm{d} \zeta .
$$

Let $\mathrm{F}_{\sigma 1}^{\mathrm{lw}}$ and $\left\langle\mathrm{F}_{\sigma 2}^{\mathrm{lw}}\right\rangle$ (note the different font) be solutions of (66) and (79) satisfying (88) or any other set of conditions that fix the component that belongs to the kernel. Then, any solution of (66) and (79) is of the form $\mathrm{F}_{\sigma 1}^{\mathrm{lw}}+h_{\sigma 1},\left\langle\mathrm{~F}_{\sigma 2}^{\mathrm{lw}}\right\rangle+h_{\sigma 2}$. When introduced into (85) and (86) one finds

$\sum_{\sigma} \frac{Z_{\sigma}}{\lambda_{\sigma}} n_{\sigma 1}+\sum_{\sigma} \frac{Z_{\sigma}}{\lambda_{\sigma}} \int B(\boldsymbol{r}) \mathrm{F}_{\sigma 1}^{\mathrm{lw}}(\boldsymbol{r}, u, \mu, t) \mathrm{d} u \mathrm{~d} \mu \mathrm{d} \theta=0$

and

$$
\begin{aligned}
\sum_{\sigma} & \frac{Z_{\sigma}}{\lambda_{\sigma}^{2}} n_{\sigma 2} \\
& +\sum_{\sigma} \frac{Z_{\sigma}}{\lambda_{\sigma}^{2}}\left[\int\left(B\left\langle\mathrm{~F}_{\sigma 2}^{\mathrm{w}}\right\rangle+u \hat{\boldsymbol{b}} \cdot\left(\nabla_{r} \times \hat{\boldsymbol{b}}\right) F_{\sigma 1}^{\mathrm{lw}}\right) \mathrm{d} u \mathrm{~d} \mu \mathrm{d} \theta\right. \\
& -\hat{\boldsymbol{b}} \cdot\left(\nabla_{r} \times \boldsymbol{K}\right) \frac{n_{\sigma} T_{\sigma}}{B^{2}}+\nabla_{r} \cdot\left(\frac{3}{2} \frac{\nabla_{r_{\perp}} B}{B^{3}} n_{\sigma} T_{\sigma}\right) \\
& +\frac{1}{2} \nabla_{r} \nabla_{r}:\left((\stackrel{\leftrightarrow}{\boldsymbol{I}}-\hat{\boldsymbol{b}} \hat{\boldsymbol{b}}) \frac{n_{\sigma} T_{\sigma}}{B^{2}}\right) \\
& \left.+\nabla_{\boldsymbol{r}} \cdot\left(\frac{n_{\sigma} T_{\sigma}}{B^{2}} \boldsymbol{\kappa}+\frac{Z_{\sigma} n_{\sigma}}{B^{2}} \nabla_{r} \varphi_{0}\right)\right]=0
\end{aligned}
$$

The electrostatic potential $\varphi_{0}$ enters equation (93) but it can never be determined from it. The first and second-order pieces of the long-wavelength quasineutrality equation simply give constraints on the corrections $n_{\sigma 1}$ and $n_{\sigma 2}$. And these corrections cannot be simply set equal to zero: in section 5.2 a transport equation determining $n_{\sigma}$ will be derived as a solvability condition for equation (79). Analogously, $n_{\sigma j}$ would be determined by a transport equation obtained as a solvability conditions for a higher-order piece of the FokkerPlanck equation.

It is important to note that the lowest-order radial electric field cannot be determined from the quasineutrality equation but the lowest-order pieces of the electric field parallel to the flux surface are determined by equations (92) and (93). This 
becomes obvious when one writes (92) and (93) in terms of the non-adiabatic pieces of the distribution function and then acts with the operators $\partial_{\Theta}$ and $\partial_{\zeta}$. In this way, $\partial_{\Theta} \varphi_{1}^{\mathrm{lw}}, \partial_{\zeta} \varphi_{1}^{\mathrm{lw}}$, $\partial_{\Theta} \varphi_{2}^{\mathrm{lw}}$, and $\partial_{\zeta} \varphi_{2}^{\mathrm{lw}}$ are obtained. The key is to recall that $n_{\sigma j}$ depends only on $\psi$. Of course, $\varphi_{1}^{\text {lw }}$ and $\varphi_{2}^{\text {lw }}$ are determined up to an arbitrary, additive function of $\psi$, that can be absorbed by redefining the corrections $n_{\sigma 1}$ and $n_{\sigma 2}$. Without loss of generality, we take

$$
\left\langle\varphi_{1}^{\mathrm{lw}}\right\rangle_{\psi}=0
$$

and

$$
\left\langle\varphi_{2}^{\mathrm{lw}}\right\rangle_{\psi}=0
$$

therefore fixing the ambiguity.

\section{Transport equations as solvability conditions of the Fokker-Planck equations}

The understanding of transport equations as solvability conditions of kinetic equations dates back to the works by Chapman and Enskog on gases [50]. In our context, when we speak about solvability conditions of the Fokker-Planck equations we mean the following: (66) and (79) are equations for $F_{\sigma 1}^{\mathrm{lw}}$ and $\left\langle F_{\sigma 2}^{\mathrm{lw}}\right\rangle$ but, in general, they cannot be solved for arbitrary values of the lower-order quantities in them. The constraints that the lower-order quantities entering (66) and (79) must satisfy are called solvability conditions of the first and second-order Fokker-Planck equations.

In appendix $M$ of [14] an exhaustive and general computation of the solvability conditions of the Fokker-Planck equations for strongly magnetized plasmas was given. Let us apply the general results of that appendix to the stellarator problem. Equation (73) can be rewritten in the form

$$
\begin{aligned}
\tau_{\sigma} \lambda_{\sigma}^{-1} & \left(u \hat{\boldsymbol{b}} \cdot \nabla_{\boldsymbol{R}}-\mu \hat{\boldsymbol{b}} \cdot \nabla_{\boldsymbol{R}} B \partial_{u}\right) G_{\sigma 1}^{\mathrm{lw}} \\
& -\tau_{\sigma} \sum_{\sigma^{\prime}}\left(\mathcal{T}_{\sigma, 0}^{*} C_{\sigma \sigma^{\prime}}\left[\lambda_{\sigma}^{-1} \mathcal{T}_{\sigma, 0}^{-1 *} G_{\sigma 1}^{\mathrm{lw}}, \mathcal{T}_{\sigma^{\prime}, 0}^{-1 *} F_{\sigma^{\prime} 0}\right]\right. \\
& \left.+\mathcal{T}_{\sigma, 0}^{*} C_{\sigma \sigma^{\prime}}\left[\mathcal{T}_{\sigma, 0}^{-1 *} F_{\sigma 0}, \lambda_{\sigma^{\prime}}^{-1} \mathcal{T}_{\sigma^{\prime}, 0}^{-1 *} G_{\sigma^{\prime} 1}^{\mathrm{lw}}\right]\right)=\tau_{\sigma} \lambda_{\sigma}^{-1} R_{\sigma 1},
\end{aligned}
$$

where

$$
R_{\sigma 1}=-\left(\frac{Z_{\sigma}}{T_{\sigma}} \partial_{\psi} \varphi_{0}+\Upsilon_{\sigma}\right) \boldsymbol{v}_{M} \cdot \nabla_{\boldsymbol{R}} \psi F_{\sigma 0}
$$

As for the second-order Fokker-Planck equation, we use the form given in (D.9), which can be expressed as

$$
\begin{aligned}
\tau_{\sigma} \lambda_{\sigma}^{-2} & \left(u \hat{\boldsymbol{b}} \cdot \nabla_{\boldsymbol{R}}-\mu \hat{\boldsymbol{b}} \cdot \nabla_{\boldsymbol{R}} B \partial_{u}\right) G_{\sigma 2}^{\mathrm{lw}} \\
& -\tau_{\sigma} \sum_{\sigma^{\prime}}\left(\mathcal{T}_{\sigma, 0}^{*} C_{\sigma \sigma^{\prime}}\left[\lambda_{\sigma}^{-2} \mathcal{T}_{\sigma, 0}^{-1 *} G_{\sigma 2}^{\mathrm{lw}}, \mathcal{T}_{\sigma^{\prime}, 0}^{-1 *} F_{\sigma^{\prime} 0}\right]\right. \\
& \left.+\mathcal{T}_{\sigma, 0}^{*} C_{\sigma \sigma^{\prime}}\left[\mathcal{T}_{\sigma, 0}^{-1 *} F_{\sigma 0}, \lambda_{\sigma^{\prime}}^{-2} \mathcal{T}_{\sigma^{\prime}, 0}^{-1 *} G_{\sigma^{\prime} 2}^{\mathrm{lw}}\right]\right)=\tau_{\sigma} \lambda_{\sigma}^{-2} R_{\sigma 2}
\end{aligned}
$$

with

$$
\begin{aligned}
& R_{\sigma 2}=-\frac{\lambda_{\sigma}^{2}}{\tau_{\sigma}} \partial_{\epsilon_{\mathrm{s}}^{2} t} F_{\sigma 0}-\frac{1}{B} \nabla_{\boldsymbol{R}} \cdot\left[\left(\mu \hat{\boldsymbol{b}} \times \nabla_{\boldsymbol{R}} B\right.\right. \\
& \left.+u^{2} \nabla_{\boldsymbol{R}} \times \hat{\boldsymbol{b}}+Z_{\sigma} \hat{\boldsymbol{b}} \times \nabla_{\boldsymbol{R}} \varphi_{0}\right) G_{\sigma 1}^{\mathrm{lw}} \\
& \left.-Z_{\sigma} \lambda_{\sigma} \varphi_{1}^{\mathrm{lw}} \hat{\boldsymbol{b}} \times \nabla_{\boldsymbol{R}} \psi\left(\Upsilon_{\sigma}+\frac{Z_{\sigma}}{T_{\sigma}} \partial_{\psi} \varphi_{0}\right) F_{\sigma 0}\right] \\
& +\frac{1}{B} \partial_{u}\left[u\left(\nabla_{\boldsymbol{R}} \times \hat{\boldsymbol{b}}\right) \cdot\left(\mu \nabla_{\boldsymbol{R}} B+Z_{\sigma} \nabla_{\boldsymbol{R}} \varphi_{0}\right) G_{\sigma 1}^{\mathrm{lw}}\right. \\
& -\frac{Z_{\sigma} \lambda_{\sigma} \varphi_{1}^{\mathrm{lw}}}{u} \mu\left(\hat{\boldsymbol{b}} \times \nabla_{\boldsymbol{R}} \psi\right) \\
& \left.\cdot \nabla_{\boldsymbol{R}} B\left(\Upsilon_{\sigma}+\frac{Z_{\sigma}}{T_{\sigma}} \partial_{\psi} \varphi_{0}\right) F_{\sigma 0}\right] \\
& +\frac{u \mu}{B}\left(\nabla_{\boldsymbol{R}} \times \boldsymbol{K}\right)_{\perp} \cdot \nabla_{\boldsymbol{R}} F_{\sigma 0} \\
& -\mu\left(\nabla_{\boldsymbol{R}} \times \boldsymbol{K}\right)_{\perp} \cdot\left(\mu \nabla_{\boldsymbol{R}} B+Z_{\sigma} \nabla_{\boldsymbol{R}} \varphi_{0}\right) \partial_{u} F_{\sigma 0} \\
& -\frac{Z_{\sigma} \lambda_{\sigma}}{B}\left[\nabla_{\boldsymbol{R}} \cdot\left(\hat{\boldsymbol{b}} \times \nabla_{\boldsymbol{R}_{\perp} / \epsilon_{\sigma}}\left\langle\phi_{\sigma 1}^{\mathrm{sw}}\right\rangle F_{\sigma 1}^{\mathrm{sw}}\right)\right]^{\mathrm{lw}} \\
& +Z_{\sigma} \lambda_{\sigma} \partial_{u}\left[\left(\hat{\boldsymbol{b}} \cdot \nabla_{\boldsymbol{R}}\left\langle\phi_{\sigma 1}^{\mathrm{sw}}\right\rangle\right.\right. \\
& \left.\left.+\frac{u}{B}(\hat{\boldsymbol{b}} \times \kappa) \cdot \nabla_{\boldsymbol{R}_{\perp} / \epsilon_{\sigma}}\left\langle\phi_{\sigma 1}^{\mathrm{sw}}\right\rangle\right) F_{\sigma 1}^{\mathrm{sw}}\right]^{\mathrm{lw}} \\
& +\partial_{u}\left[-\frac{Z_{\sigma} \lambda_{\sigma} \varphi_{1}^{\mathrm{lw}}}{u} \sum_{\sigma^{\prime}}\left\langle\mathcal{T}_{\sigma, 0}^{*} C_{\sigma \sigma^{\prime}}^{(1) \mathrm{lw}}\right\rangle\right] \\
& +\frac{u}{B} \hat{\boldsymbol{b}} \cdot \nabla_{\boldsymbol{R}} \times \hat{\boldsymbol{b}} \sum_{\sigma^{\prime}}\left\langle\mathcal{T}_{\sigma, 0}^{*} C_{\sigma \sigma^{\prime}}^{(1) \mathrm{lw}}\right\rangle \\
& +\sum_{\sigma^{\prime}}\left\langle\left[\mathcal{T}_{\sigma, 1}^{*} C_{\sigma \sigma^{\prime}}^{(1)}\right]^{\mathrm{lw}}\right\rangle+\sum_{\sigma^{\prime}}\left\langle\mathcal{T}_{\sigma, 0}^{*} \mathbb{C}_{\sigma \sigma^{\prime}}\right\rangle .
\end{aligned}
$$

The term $\mathbb{C}_{\sigma \sigma^{\prime}}$ is defined in (B.12). As shown in appendix $\mathrm{M}$ of [14], given the form of equations (97) and (99), the solvability conditions are

$$
\left\langle\tau_{\sigma} \lambda_{\sigma}^{-j} \int B R_{\sigma j} \mathrm{~d} u \mathrm{~d} \mu \mathrm{d} \theta\right\rangle_{\psi}=0, \quad \text { for each } \sigma
$$

and

$\left\langle\sum_{\sigma} \tau_{\sigma} \lambda_{\sigma}^{-j} \int B\left(u^{2} / 2+\mu B\right) R_{\sigma j} \mathrm{~d} u \mathrm{~d} \mu \mathrm{d} \theta\right\rangle_{\psi}=0$,

with $j=1,2$.

\subsection{Triviality of the solvability condition of the first-order Fokker-Planck equations}

We start by showing that the first-order Fokker-Planck equations do not give any constraint on lower-order quantities, i.e. we prove that (100) and (101) turn out to yield trivial conditions when $R_{\sigma 1}$ is given by (97). In order to conclude 
that (100) and (101) vanish identically for $j=1$ we need to prove the identity

$$
\left\langle\int B h(\psi, \varepsilon) v_{M} \cdot \nabla_{R} \psi \mathrm{d} u \mathrm{~d} \mu \mathrm{d} \theta\right\rangle_{\psi} \equiv 0,
$$

for any function $h$ of the kinetic energy $\varepsilon$ and the flux-surface label $\psi$. Here, $\varepsilon=u^{2} / 2+\mu B$. The radial component of the magnetic drift velocity can be written as

$$
\begin{gathered}
\boldsymbol{v}_{M} \cdot \nabla_{\boldsymbol{R}} \psi=\frac{u^{2}}{B}\left(\nabla_{\boldsymbol{R}} \times \hat{\boldsymbol{b}}\right) \cdot \nabla_{\boldsymbol{R}} \psi \\
+\frac{\mu}{B}\left(\hat{\boldsymbol{b}} \times \nabla_{\boldsymbol{R}} B\right) \cdot \nabla_{\boldsymbol{R}} \psi .
\end{gathered}
$$

Then, we have

$$
\begin{gathered}
\left\langle\int B h(\psi, \varepsilon) \boldsymbol{v}_{M} \cdot \nabla_{\boldsymbol{R}} \psi \mathrm{d} u \mathrm{~d} \mu \mathrm{d} \theta\right\rangle_{\psi} \\
=\left\langle\nabla_{\boldsymbol{R}} \cdot \int u^{2} h \hat{\boldsymbol{b}} \times \nabla_{\boldsymbol{R}} \psi \mathrm{d} u \mathrm{~d} \mu \mathrm{d} \theta\right\rangle_{\psi} \\
\quad-\left\langle\int \left(\mu h\left(\hat{\boldsymbol{b}} \times \nabla_{\boldsymbol{R}} \psi\right) \cdot \nabla_{\boldsymbol{R}} B\right.\right. \\
\left.\left.+u^{2}\left(\hat{\boldsymbol{b}} \times \nabla_{\boldsymbol{R}} \psi\right) \cdot \nabla_{\boldsymbol{R}} h\right) \mathrm{~d} u \mathrm{~d} \mu \mathrm{d} \theta\right\rangle_{\psi},
\end{gathered}
$$

where we have used that $\nabla_{\boldsymbol{R}} \cdot\left(\boldsymbol{A}_{1} \times \boldsymbol{A}_{2}\right)=\left(\nabla_{\boldsymbol{R}} \times \boldsymbol{A}_{1}\right) \cdot \boldsymbol{A}_{2}-$ $\left(\nabla_{\boldsymbol{R}} \times \boldsymbol{A}_{2}\right) \cdot \boldsymbol{A}_{1}$ for any two vector fields $\boldsymbol{A}_{1}$ and $\boldsymbol{A}_{2}$, and that the curl of the gradient of a function is zero. Noting that $u\left(\hat{\boldsymbol{b}} \times \nabla_{\boldsymbol{R}} \psi\right) \cdot \nabla_{\boldsymbol{R}} h=\mu\left(\hat{\boldsymbol{b}} \times \nabla_{\boldsymbol{R}} \psi\right) \cdot \nabla_{\boldsymbol{R}} B \partial_{u} h$ and integrating by parts in $u$ the last term of (104), we find that

$$
\begin{aligned}
& \left\langle\int \left(\mu h\left(\hat{\boldsymbol{b}} \times \nabla_{\boldsymbol{R}} \psi\right) \cdot \nabla_{\boldsymbol{R}} B\right.\right. \\
& \left.\left.\quad+u^{2}\left(\hat{\boldsymbol{b}} \times \nabla_{\boldsymbol{R}} \psi\right) \cdot \nabla_{\boldsymbol{R}} h\right) \mathrm{~d} u \mathrm{~d} \mu \mathrm{d} \theta\right\rangle_{\psi}=0 .
\end{aligned}
$$

The first term on the right side of (104) also vanishes, as can be easily seen by employing the formula

$$
\left\langle\nabla_{\boldsymbol{R}} \cdot \boldsymbol{A}\right\rangle_{\psi}=\frac{1}{V^{\prime}(\psi)} \partial_{\psi}\left\langle V^{\prime}(\psi) \boldsymbol{A} \cdot \nabla_{\boldsymbol{R}} \psi\right\rangle_{\psi}
$$

for the flux-surface average of the divergence of a vector field $\boldsymbol{A}$. Here, $V^{\prime}(\psi)$ is the derivative with respect to $\psi$ of the volume defined in (91).

Therefore, we have proven (102) and learnt that the firstorder Fokker-Planck equations do not impose any constraints on lower-order quantities. The solvability conditions (100) and (101) for $j=2$ are non-trivial; actually they are transport equations for the particle density of each species and for the total kinetic energy, respectively. We work them out in the next subsections.

\subsection{Transport equation for particle density}

The transport equation for particle density of species $\sigma$ is obtained from (100) when $j=2$ and $R_{\sigma 2}$ is given by (99). In appendix $E$ it is shown that the result is

$$
\begin{aligned}
& \partial_{\epsilon_{\mathrm{s}}^{2} t} n_{\sigma}(\psi, t) \\
& +V^{\prime-1} \partial_{\psi}\left\langle V^{\prime} \frac{\tau_{\sigma}}{\lambda_{\sigma}^{2}} \int B \boldsymbol{v}_{M} \cdot \nabla_{\boldsymbol{R}} \psi G_{\sigma 1}^{\mathrm{lw}} \mathrm{d} u \mathrm{~d} \mu \mathrm{d} \theta\right\rangle_{\psi} \\
& =V^{\prime-1} \partial_{\psi}\left\langleV ^ { \prime } \int \left\{-\left[\left(\hat{\boldsymbol{b}} \times \nabla_{\boldsymbol{R}_{\perp} / \epsilon_{\sigma}}\left\langle\phi_{\sigma 1}^{\mathrm{sw}}\right\rangle F_{\sigma 1}^{\mathrm{sw}}\right)\right]^{\mathrm{lw}} \cdot \nabla_{\boldsymbol{R}} \psi\right.\right. \\
& \left.\left.+\frac{\tau_{\sigma}}{\lambda_{\sigma}^{2}} \sum_{\sigma^{\prime}}\left\langle B \boldsymbol{\rho} \cdot \nabla_{\boldsymbol{R}} \psi \mathcal{T}_{\sigma, 0}^{*} C_{\sigma \sigma^{\prime}}^{(1) \mathrm{lw}}\right\rangle\right\} \mathrm{d} u \mathrm{~d} \mu \mathrm{d} \theta\right\rangle_{\psi}
\end{aligned}
$$

Observe that only the non-adiabatic piece of $F_{\sigma 1}^{\mathrm{lw}}, G_{\sigma 1}^{\mathrm{lw}}=$ $F_{\sigma 1}^{\mathrm{lw}}+\left(Z_{\sigma} \lambda_{\sigma} / T_{\sigma}\right) \varphi_{1}^{\mathrm{lw}} F_{\sigma 0}$, contributes to particle transport. The quantity $C_{\sigma \sigma^{\prime}}^{(1) \mathrm{lw}}$ is defined in B.1.

\subsection{Transport equation for energy}

The solvability condition (101) for $j=2$ and $R_{\sigma 2}$ corresponding to (99) gives a transport equation for the total energy. Its computation requires some algebra, and this is done in appendix F. The result is

$$
\begin{aligned}
\partial_{\epsilon_{\mathrm{s}}^{2} t} \sum_{\sigma} & \frac{3}{2} n_{\sigma} T_{\sigma} \\
& +\frac{1}{V^{\prime}} \partial_{\psi}\left\langleV ^ { \prime } \int B ( u ^ { 2 } / 2 + \mu B ) \left\{\sum_{\sigma} \frac{\tau_{\sigma}}{\lambda_{\sigma}^{2}} \boldsymbol{v}_{M} \cdot \nabla_{\boldsymbol{R}} \psi G_{\sigma 1}^{\mathrm{lw}}\right.\right. \\
& +\frac{1}{B} \sum_{\sigma}\left[\left(\hat{\boldsymbol{b}} \times \nabla_{\boldsymbol{R}_{\perp} / \epsilon_{\sigma}}\left\langle\phi_{\sigma 1}^{\mathrm{sw}}\right\rangle \cdot \nabla_{\boldsymbol{R}} \psi F_{\sigma 1}^{\mathrm{sw}}\right)\right]^{\mathrm{lw}} \\
& \left.\left.-\sum_{\sigma, \sigma^{\prime}} \frac{\tau_{\sigma}}{\lambda_{\sigma}^{2}}\left\langle\boldsymbol{\rho} \cdot \nabla_{\boldsymbol{R}} \psi \mathcal{T}_{\sigma, 0}^{*} C_{\sigma \sigma^{\prime}}^{(1) \mathrm{lw}}\right\rangle\right\} \mathrm{d} u \mathrm{~d} \mu \mathrm{d} \theta\right\rangle_{\psi}=0
\end{aligned}
$$

Again, only the non-adiabatic piece of $F_{\sigma 1}^{\text {lw }}$ contributes to energy transport.

\subsection{Ambipolarity condition}

In section 5.2 we have computed a transport equation, (107), for the particle density of each species. This is a solvability condition of the Fokker-Planck equation to $O\left(\epsilon_{\mathrm{s}}^{2}\right)$. But we must recall that the quasineutrality equation to lowest order, (84), imposes the constraint

$$
\sum_{\sigma} Z_{\sigma} n_{\sigma}(\psi, t)=0,
$$

for every $\psi$ and $t$. Hence,

$$
\partial_{\epsilon_{\mathrm{s}}^{2} t} \sum_{\sigma} Z_{\sigma} n_{\sigma}(\psi, t)=0
$$


should also hold for every $\psi$ and $t$. The question is whether the density transport equations (107) automatically imply $\partial_{\epsilon_{\mathrm{s}}^{2} t} \sum_{\sigma} Z_{\sigma} n_{\sigma}(\psi, t) \equiv 0$ or, on the contrary, the vanishing of $\partial_{\epsilon_{s}^{2} t} \sum_{\sigma} Z_{\sigma} n_{\sigma}(\psi, t)$, needed for consistency, yields an equation that sets new constraints on lowest-order quantities. To find the answer we proceed to multiply equation (107) by $Z_{\sigma}$ and sum over $\sigma$. The last term vanishes due to momentum conservation by the collision operator (see (A.9)). The contribution of the first term on the right side of (107) to $\partial_{\epsilon_{\mathrm{s}}^{2} t} \sum_{\sigma} Z_{\sigma} n_{\sigma}$ also vanishes, irrespective of the magnetic geometry, i.e.

$$
\begin{aligned}
& \sum_{\sigma} Z_{\sigma}\left\langle\int B\left[F_{\sigma 1}^{\mathrm{sw}}\left(\nabla_{\boldsymbol{R}_{\perp} / \epsilon_{\sigma}}\left\langle\phi_{\sigma 1}^{\mathrm{sw}}\right\rangle \times \hat{\boldsymbol{b}}\right) \cdot \nabla_{\boldsymbol{R}} \psi\right]^{\mathrm{lw}}\right. \\
& \times \mathrm{d} u \mathrm{~d} \mu \mathrm{d} \theta\rangle_{\psi}=0 .
\end{aligned}
$$

This was proven in [7,51] and also in section 5.3 of [14]. As a consequence, we have

$$
\begin{aligned}
& \partial_{\epsilon_{\mathrm{s}}^{2} t} \sum_{\sigma} Z_{\sigma} n_{\sigma}(\psi, t) \\
& +\sum_{\sigma} V^{\prime-1} \partial_{\psi}\left\langle V^{\prime} \frac{1}{\lambda_{\sigma}} \int B \boldsymbol{v}_{M} \cdot \nabla_{\boldsymbol{R}} \psi G_{\sigma 1}^{\mathrm{lw}} \mathrm{d} u \mathrm{~d} \mu \mathrm{d} \theta\right\rangle_{\psi}=0 .
\end{aligned}
$$

Of course, consistency with (109) enforces

$\sum_{\sigma} V^{\prime-1} \partial_{\psi}\left\langle V^{\prime} \frac{1}{\lambda_{\sigma}} \int B \boldsymbol{v}_{M} \cdot \nabla_{\boldsymbol{R}} \psi G_{\sigma 1}^{\mathrm{lw}} \mathrm{d} u \mathrm{~d} \mu \mathrm{d} \theta\right\rangle_{\psi}=0$.

Regularity at $\psi=0$ makes (113) equivalent to

$$
\sum_{\sigma}\left\langle\frac{1}{\lambda_{\sigma}} \int B \boldsymbol{v}_{M} \cdot \nabla_{\boldsymbol{R}} \psi G_{\sigma 1}^{\mathrm{lw}} \mathrm{d} u \mathrm{~d} \mu \mathrm{d} \theta\right\rangle_{\psi}=0
$$

which is the standard neoclassical ambipolarity condition. This is the lowest-order piece of the equation

$$
\left\langle\boldsymbol{J} \cdot \nabla_{\boldsymbol{R}} \psi\right\rangle_{\psi}=0,
$$

where $\boldsymbol{J}$ is the electric current density.

\section{When does the ambipolarity condition add any information?}

In this section we want to discuss the circumstances under which (114) is actually an equation that imposes new conditions on lowest-order quantities. The general answer to the above question has already been given by Helander and Simakov in [1]. Here, we give our own version of the proof for completeness.

Since the temperature profile is the same for all species, in this section we remove the subindex $\sigma$, and $T_{\sigma} \equiv T$. At every point of phase space $(\psi, \Theta, \zeta, u, \mu, \theta)$, the function $G_{\sigma 1}^{\mathrm{lw}}$, determined by (73), is a linear combination of the gradients of the profiles at $\psi$. Namely,

$$
G_{\sigma 1}^{\mathrm{lw}}=\partial_{\psi} \varphi_{0} g_{\sigma}+\sum_{\gamma} \partial_{\psi} n_{\gamma} \check{h}_{\sigma \gamma}+\partial_{\psi} T l_{\sigma},
$$

for some phase-space functions $g_{\sigma}, \breve{h}_{\sigma \gamma}, l_{\sigma}$ that do not depend on the profile gradients and whose defining equations are given below, in equations (119), (120), and (121). In (116) the index $\gamma$ runs over all species. Denote by $N$ the number of different species. There are not $N$ independent density gradients, but $N-1$ due to the constraint $\sum_{\sigma} Z_{\sigma} n_{\sigma}(\psi, t)=0$. For example, we can eliminate the gradient of the electron density, $n_{\mathrm{e}}$, by using

$$
\partial_{\psi} n_{\mathrm{e}}(\psi, t)=\sum_{\sigma \neq e} Z_{\sigma} \partial_{\psi} n_{\sigma}(\psi, t)
$$

It is conceptually clearer to express $G_{\sigma 1}^{\mathrm{lw}}$ in terms of the $N+1$ independent gradients,

$$
G_{\sigma 1}^{\mathrm{lw}}=\partial_{\psi} \varphi_{0} g_{\sigma}+\sum_{\gamma \neq e} \partial_{\psi} n_{\gamma} h_{\sigma \gamma}+\partial_{\psi} T l_{\sigma}
$$

where $h_{\sigma \gamma}:=\check{h}_{\sigma \gamma}+Z_{\gamma} \check{h}_{\sigma e}$ is defined only for $\gamma \neq e$. From (73) and (118) we can easily find the equations that determine the coefficients of the gradients in (118),

$$
\begin{aligned}
& \left(u \hat{\boldsymbol{b}} \cdot \nabla_{\boldsymbol{R}}-\mu \hat{\boldsymbol{b}} \cdot \nabla_{\boldsymbol{R}} B \partial_{u}\right) g_{\sigma}+\frac{Z_{\sigma}}{T} \boldsymbol{v}_{M} \cdot \nabla_{\boldsymbol{R}} \psi F_{\sigma 0} \\
& =\sum_{\sigma^{\prime}} \mathcal{T}_{\sigma, 0}^{*} C_{\sigma \sigma^{\prime}}\left[\mathcal{T}_{\sigma, 0}^{-1 *} g_{\sigma}, \mathcal{T}_{\sigma^{\prime}, 0}^{-1 *} F_{\sigma^{\prime} 0}\right] \\
& \quad+\sum_{\sigma^{\prime}} \frac{\lambda_{\sigma}}{\lambda_{\sigma^{\prime}}} \mathcal{T}_{\sigma, 0}^{*} C_{\sigma \sigma^{\prime}}\left[\mathcal{T}_{\sigma, 0}^{-1 *} F_{\sigma 0}, \mathcal{T}_{\sigma^{\prime}, 0}^{-1 *} g_{\sigma^{\prime}}\right],
\end{aligned}
$$

$$
\begin{aligned}
& \left(u \hat{\boldsymbol{b}} \cdot \nabla_{\boldsymbol{R}}-\mu \hat{\boldsymbol{b}} \cdot \nabla_{\boldsymbol{R}} B \partial_{u}\right) h_{\sigma \gamma} \\
& \quad+\frac{1}{n_{\sigma}}\left(\delta_{\sigma \gamma}+Z_{\gamma} \delta_{\sigma e}\right) \boldsymbol{v}_{M} \cdot \nabla_{\boldsymbol{R}} \psi F_{\sigma 0} \\
& =\sum_{\sigma^{\prime}} \mathcal{T}_{\sigma, 0}^{*} C_{\sigma \sigma^{\prime}}\left[\mathcal{T}_{\sigma, 0}^{-1 *} h_{\sigma \gamma}, \mathcal{T}_{\sigma^{\prime}, 0}^{-1 *} F_{\sigma^{\prime} 0}\right] \\
& \quad+\sum_{\sigma^{\prime}} \frac{\lambda_{\sigma}}{\lambda_{\sigma^{\prime}}} \mathcal{T}_{\sigma, 0}^{*} C_{\sigma \sigma^{\prime}}\left[\mathcal{T}_{\sigma, 0}^{-1 *} F_{\sigma 0}, \mathcal{T}_{\sigma^{\prime}, 0}^{-1 *} h_{\sigma^{\prime} \gamma}\right],
\end{aligned}
$$

and

$$
\begin{aligned}
(u \hat{\boldsymbol{b}} & \left.\cdot \nabla_{\boldsymbol{R}}-\mu \hat{\boldsymbol{b}} \cdot \nabla_{\boldsymbol{R}} B \partial_{u}\right) l_{\sigma} \\
& +\left(\frac{u^{2} / 2+\mu B}{T_{\sigma}}-\frac{3}{2}\right) \frac{1}{T} \boldsymbol{v}_{M} \cdot \nabla_{\boldsymbol{R}} \psi F_{\sigma 0} \\
= & \sum_{\sigma^{\prime}} \mathcal{T}_{\sigma, 0}^{*} C_{\sigma \sigma^{\prime}}\left[\mathcal{T}_{\sigma, 0}^{-1 *} l_{\sigma}, \mathcal{T}_{\sigma^{\prime}, 0}^{-1 *} F_{\sigma^{\prime} 0}\right] \\
& +\sum_{\sigma^{\prime}} \frac{\lambda_{\sigma}}{\lambda_{\sigma^{\prime}}} \mathcal{T}_{\sigma, 0}^{*} C_{\sigma \sigma^{\prime}}\left[\mathcal{T}_{\sigma, 0}^{-1 *} F_{\sigma 0}, \mathcal{T}_{\sigma^{\prime}, 0}^{-1 *} l_{\sigma^{\prime}}\right] .
\end{aligned}
$$

Equations (119), (120) and (121) have a kernel (see section 4). In order to choose particular solutions $g_{\sigma}, h_{\sigma \gamma}$ and $l_{\sigma}$, we 
impose

$\left\langle\int B g_{\sigma} \mathrm{d} u \mathrm{~d} \mu \mathrm{d} \theta\right\rangle_{\psi}=0$ for every $\sigma$,

$\left\langle\sum_{\sigma} \frac{1}{\lambda_{\sigma}} \int B\left(u^{2} / 2+\mu B\right) g_{\sigma} \mathrm{d} u \mathrm{~d} \mu \mathrm{d} \theta\right\rangle_{\psi}=0 ;$

$\left\langle\int B h_{\sigma \gamma} \mathrm{d} u \mathrm{~d} \mu \mathrm{d} \theta\right\rangle_{\psi}=0$ for every $\sigma, \gamma$,

$\left\langle\sum_{\sigma} \frac{1}{\lambda_{\sigma}} \int B\left(u^{2} / 2+\mu B\right) h_{\sigma \gamma} \mathrm{d} u \mathrm{~d} \mu \mathrm{d} \theta\right\rangle_{\psi}=0$

for every $\gamma$;

$\left\langle\int B l_{\sigma} \mathrm{d} u \mathrm{~d} \mu \mathrm{d} \theta\right\rangle_{\psi}=0$ for every $\sigma$

and

$\left\langle\sum_{\sigma} \frac{1}{\lambda_{\sigma}} \int B\left(u^{2} / 2+\mu B\right) l_{\sigma} \mathrm{d} u \mathrm{~d} \mu \mathrm{d} \theta\right\rangle_{\psi}=0$.

Other conditions are possible, but the radial current (114) will not depend on the conditions chosen to fix the solutions of (119), (120) and (121).

Now, let us write the ambipolarity condition (114) in terms of $g_{\sigma}, h_{\sigma \gamma}$ and $l_{\sigma}$ :

$$
\begin{aligned}
& \partial_{\psi} \varphi_{0} \sum_{\sigma}\left\langle\frac{1}{\lambda_{\sigma}} \int B \boldsymbol{v}_{M} \cdot \nabla_{\boldsymbol{R}} \psi g_{\sigma} \mathrm{d} u \mathrm{~d} \mu \mathrm{d} \theta\right\rangle_{\psi} \\
& \quad+\sum_{\substack{\sigma \\
\gamma \neq e}} \partial_{\psi} n_{\gamma}\left\langle\frac{1}{\lambda_{\sigma}} \int B \boldsymbol{v}_{M} \cdot \nabla_{\boldsymbol{R}} \psi h_{\sigma \gamma} \mathrm{d} u \mathrm{~d} \mu \mathrm{d} \theta\right\rangle_{\psi} \\
& +\partial_{\psi} T \sum_{\sigma}\left\langle\frac{1}{\lambda_{\sigma}} \int B \boldsymbol{v}_{M} \cdot \nabla_{\boldsymbol{R}} \psi l_{\sigma} \mathrm{d} u \mathrm{~d} \mu \mathrm{d} \theta\right\rangle_{\psi}=0 .
\end{aligned}
$$

We call the system intrinsically ambipolar if all of the coefficients of the $N+1$ independent gradients in (125) vanish. That is, if

$\sum_{\sigma}\left\langle\frac{1}{\lambda_{\sigma}} \int B \boldsymbol{v}_{M} \cdot \nabla_{\boldsymbol{R}} \psi g_{\sigma} \mathrm{d} u \mathrm{~d} \mu \mathrm{d} \theta\right\rangle_{\psi}=0$,

$\sum_{\sigma}\left\langle\frac{1}{\lambda_{\sigma}} \int B \boldsymbol{v}_{M} \cdot \nabla_{\boldsymbol{R}} \psi h_{\sigma \gamma} \mathrm{d} u \mathrm{~d} \mu \mathrm{d} \theta\right\rangle_{\psi}=0$,

for every $\gamma \neq \mathrm{e}$,

and

$\sum_{\sigma}\left\langle\frac{1}{\lambda_{\sigma}} \int B \boldsymbol{v}_{M} \cdot \nabla_{\boldsymbol{R}} \psi l_{\sigma} \mathrm{d} u \mathrm{~d} \mu \mathrm{d} \theta\right\rangle_{\psi}=0$.

This is another way of saying that an intrinsically ambipolar system is one for which (114) is an identity, i.e. it is satisfied for every value of the electrostatic potential, density, and temperature gradients. Now, we want to derive necessary and sufficient conditions for intrinsically ambipolar magnetic configurations. We assume that $\left(\nabla_{\boldsymbol{R}} \times \boldsymbol{B}\right) \cdot \nabla_{\boldsymbol{R}} \psi=0$.

First, we try to find necessary conditions for intrinsic ambipolarity. The functions $g_{\sigma}$ satisfy (119). Define $\hat{g}_{\sigma}:=$ $g_{\sigma} / F_{\sigma 0}$. Multiply (119) by $Z_{\sigma}^{-1} \lambda_{\sigma}^{-1} B \hat{g}_{\sigma}$, integrate over $u, \mu, \theta$, take flux-surface average, and sum over $\sigma$ to find

$$
\begin{aligned}
& \frac{1}{T}\left\langle\sum_{\sigma} \frac{1}{\lambda_{\sigma}} \int B \boldsymbol{v}_{M} \cdot \nabla_{\boldsymbol{R}} \psi g_{\sigma} \mathrm{d} u \mathrm{~d} \mu \mathrm{d} \theta\right\rangle_{\psi} \\
& \quad=\left\langle\sum_{\sigma} \tau_{\sigma} \int B \lambda_{\sigma}^{-1} \hat{g}_{\sigma} \sum_{\sigma^{\prime}} \hat{C}_{\sigma \sigma^{\prime}}\left[\hat{g}_{\sigma}, \hat{g}_{\sigma^{\prime}}\right] \mathrm{d} u \mathrm{~d} \mu \mathrm{d} \theta\right\rangle_{\psi},
\end{aligned}
$$

where

$$
\begin{aligned}
\hat{C}_{\sigma \sigma^{\prime}} & {\left[\hat{g}_{\sigma}, \hat{g}_{\sigma^{\prime}}\right]=\mathcal{T}_{\sigma, 0}^{*} C_{\sigma \sigma^{\prime}}\left[\lambda_{\sigma}^{-1} \mathcal{T}_{\sigma, 0}^{-1 *} g_{\sigma}, \mathcal{T}_{\sigma^{\prime}, 0}^{-1 *} F_{\sigma^{\prime} 0}\right] } \\
+ & \mathcal{T}_{\sigma, 0}^{*} C_{\sigma \sigma^{\prime}}\left[\mathcal{T}_{\sigma, 0}^{-1 *} F_{\sigma 0}, \lambda_{\sigma^{\prime}}^{-1} \mathcal{T}_{\sigma^{\prime}, 0}^{-1 *} g_{\sigma^{\prime}}\right] .
\end{aligned}
$$

By hypothesis the system is instrinsically ambipolar, so (126) holds. Thus,

$\left\langle\sum_{\sigma} \tau_{\sigma} \int B \lambda_{\sigma}^{-1} \hat{g}_{\sigma} \sum_{\sigma^{\prime}} \hat{C}_{\sigma \sigma^{\prime}}\left[\hat{g}_{\sigma}, \hat{g}_{\sigma^{\prime}}\right] \mathrm{d} u \mathrm{~d} \mu \mathrm{d} \theta\right\rangle_{\psi}=0$.

Recasting (A.7) and (A.8) into non-dimensionalized variables, one readily confirms that (131) implies

$g_{\sigma}=\alpha_{0, \sigma} F_{\sigma 0}+\alpha_{1} Z_{\sigma} u F_{\sigma 0}+\alpha_{2} \lambda_{\sigma}\left(u^{2} / 2+\mu B\right) F_{\sigma 0}$,

where $\alpha_{0, \sigma}, \alpha_{1}$, and $\alpha_{2}$ are, in principle, arbitrary functions of $\boldsymbol{R}$. Conditions (122) only give $\left\langle\alpha_{0, \sigma}\right\rangle_{\psi} \equiv 0$ for every $\sigma$, and $\left\langle\alpha_{2}\right\rangle_{\psi} \equiv 0$.

Imposing that $g_{\sigma}$, i.e. the right side of (132), satisfies (119), we get

$$
\begin{aligned}
u \hat{\boldsymbol{b}} \cdot & \nabla_{\boldsymbol{R}} \alpha_{0, \sigma}+Z_{\sigma}\left(u \hat{\boldsymbol{b}} \cdot \nabla_{\boldsymbol{R}}-\mu \hat{\boldsymbol{b}} \cdot \nabla_{\boldsymbol{R}} B \partial_{u}\right)\left(\alpha_{1} u\right) \\
& +\lambda_{\sigma} u\left(u^{2} / 2+\mu B\right) \hat{\boldsymbol{b}} \cdot \nabla_{\boldsymbol{R}} \alpha_{2}+\frac{Z_{\sigma}}{T} \boldsymbol{v}_{M} \cdot \nabla_{\boldsymbol{R}} \psi=0 .
\end{aligned}
$$

This equation must hold for every $u$ and $\mu$. Setting $\mu=0$, the terms linear in $u$ give $\hat{\boldsymbol{b}} \cdot \nabla_{\boldsymbol{R}} \alpha_{0, \sigma} \equiv 0$ and the terms cubic in $u$ give $\hat{b} \cdot \nabla_{R} \alpha_{2} \equiv 0$. Therefore, we are left with

$\left(u \hat{\boldsymbol{b}} \cdot \nabla_{\boldsymbol{R}}-\mu \hat{\boldsymbol{b}} \cdot \nabla_{\boldsymbol{R}} B \partial_{u}\right)\left(\alpha_{1} u\right)+\frac{1}{T} \boldsymbol{v}_{M} \cdot \nabla_{\boldsymbol{R}} \psi=0$.

Observe that if $\boldsymbol{B}$ satisfies $\left(\nabla_{\boldsymbol{R}} \times \boldsymbol{B}\right) \cdot \nabla_{\boldsymbol{R}} \psi=0$, then

$$
\boldsymbol{v}_{M} \cdot \nabla_{\boldsymbol{R}} \psi=\frac{u^{2}+\mu B}{B^{2}}\left(\hat{\boldsymbol{b}} \times \nabla_{\boldsymbol{R}} B\right) \cdot \nabla_{\boldsymbol{R}} \psi .
$$

Hence, (134) is recast into

$u^{2} \hat{\boldsymbol{b}} \cdot \nabla_{\boldsymbol{R}} \alpha_{1}-\alpha_{1} \mu \hat{\boldsymbol{b}} \cdot \nabla_{\boldsymbol{R}} B$

$$
+\frac{1}{T} \frac{u^{2}+\mu B}{B^{2}}\left(\hat{\boldsymbol{b}} \times \nabla_{R} B\right) \cdot \nabla_{R} \psi=0 .
$$

Again, this identity must hold for every value of $u$ and $\mu$. If $\mu=0$, then

$$
\frac{1}{T} \frac{1}{B}\left(\hat{\boldsymbol{b}} \times \nabla_{R} B\right) \cdot \nabla_{R} \psi=-B \hat{\boldsymbol{b}} \cdot \nabla_{R} \alpha_{1} .
$$


If $u=0$, then

$$
\frac{1}{T} \frac{1}{B}\left(\hat{\boldsymbol{b}} \times \nabla_{\boldsymbol{R}} B\right) \cdot \nabla_{\boldsymbol{R}} \psi=\alpha_{1} \hat{\boldsymbol{b}} \cdot \nabla_{\boldsymbol{R}} B .
$$

Subtracting the last two equations one finds $\hat{\boldsymbol{b}} \cdot \nabla_{\boldsymbol{R}}\left(B \alpha_{1}\right)=0$, and consequently we can write

$$
B \alpha_{1}=-\frac{\chi}{T}
$$

for some flux function $\chi(\psi)$. Employing (139) in (138) we get a condition that is expressed solely in terms of the magnetic field:

$$
\left(\hat{\boldsymbol{b}} \times \nabla_{R} \psi\right) \cdot \nabla_{R} B=\chi(\psi) \hat{\boldsymbol{b}} \cdot \nabla_{R} B .
$$

A magnetic field satisfying (140) for some flux function $\chi$ is called quasisymmetric. Note that in order to get this necessary condition we have not employed either (127) or (128).

Now, let us show that quasisymmetry, (140), is a sufficient condition for intrinsic ambipolarity. The essential point is to note that in a quasisymmetric system with $\left(\nabla_{\boldsymbol{R}} \times \boldsymbol{B}\right) \cdot \nabla_{\boldsymbol{R}} \psi=0$, the radial magnetic drift can be written as

$$
\boldsymbol{v}_{M} \cdot \nabla_{\boldsymbol{R}} \psi=\chi(\psi)\left(u \hat{\boldsymbol{b}} \cdot \nabla_{\boldsymbol{R}}-\mu \hat{\boldsymbol{b}} \cdot \nabla_{\boldsymbol{R}} B \partial_{u}\right) \frac{u}{B} .
$$

We have to prove that (126), (127), and (128) are satisfied. Take (126). Using (141) and integration by parts,

$$
\begin{aligned}
& \sum_{\sigma}\left\langle\frac{1}{\lambda_{\sigma}} \int B \boldsymbol{v}_{M} \cdot \nabla_{\boldsymbol{R}} \psi g_{\sigma} \mathrm{d} u \mathrm{~d} \mu \mathrm{d} \theta\right\rangle_{\psi} \\
& =-\chi(\psi) \sum_{\sigma}\left(\frac{1}{\lambda_{\sigma}} \int u\left(u \hat{\boldsymbol{b}} \cdot \nabla_{\boldsymbol{R}}-\mu \hat{\boldsymbol{b}} \cdot \nabla_{\boldsymbol{R}} B \partial_{u}\right) g_{\sigma} \mathrm{d} u \mathrm{~d} \mu \mathrm{d} \theta\right\rangle_{\psi}
\end{aligned}
$$

Recall that $g_{\sigma}$ satisfies (119). Using equation (119) in (142), one sees that the last term on the left side of (119) does not contribute because it is even in $u$. The contribution of the right side of (119) gives

$$
\begin{aligned}
& \sum_{\sigma}\left\langle\frac{1}{\lambda_{\sigma}} \int B \boldsymbol{v}_{M} \cdot \nabla_{\boldsymbol{R}} \psi g_{\sigma} \mathrm{d} u \mathrm{~d} \mu \mathrm{d} \theta\right\rangle_{\psi} \\
& =-\chi(\psi) \sum_{\sigma}\left\langle\frac { 1 } { \lambda _ { \sigma } } \int u \left(\sum_{\sigma^{\prime}} \mathcal{T}_{\sigma, 0}^{*} C_{\sigma \sigma^{\prime}}\left[\mathcal{T}_{\sigma, 0}^{-1 *} g_{\sigma}, \mathcal{T}_{\sigma^{\prime}, 0}^{-1 *} F_{\sigma^{\prime} 0}\right]\right.\right. \\
& \left.\left.+\sum_{\sigma^{\prime}} \frac{\lambda_{\sigma}}{\lambda_{\sigma^{\prime}}} \mathcal{T}_{\sigma, 0}^{*} C_{\sigma \sigma^{\prime}}\left[\mathcal{T}_{0, \sigma}^{-1 *} F_{\sigma 0}, \mathcal{T}_{0, \sigma^{\prime}}^{-1 *} g_{\sigma^{\prime}}\right]\right) \mathrm{d} u \mathrm{~d} \mu \mathrm{d} \theta\right\rangle_{\psi}
\end{aligned}
$$

which is zero due to the fact that the collision operator conserves total momentum (see (A.9)). Analogous steps lead to (127) and (128).

To summarize, a magnetic configuration with $\left(\nabla_{R} \times\right.$ B) $\cdot \nabla_{R} \psi=0$ is intrinsically ambipolar if and only if it is quasisymmetric. If the stellarator is not quasisymmetric, $\varphi_{0}$ can be found from neoclassical theory. That is, from (114) and (73). However, if the stellarator is quasisymmetric, the ambipolarity condition (114) is identically satisfied to lowest order. The determination of $\varphi_{0}$ is not a neoclassical problem anymore, and one has to include both turbulent and neoclassical terms. As explained in [11] for the tokamak, a moment approach gives $\varphi_{0}$ if the second-order pieces of the distribution function and turbulent electrostatic potential are known. This is why the full second-order gyrokinetic equations are required. The long-wavelength component of the second-order Fokker-Planck and quasineutrality equations have to be solved to calculate $\varphi_{0}$. The short-wavelength component of the equations to second-order are also needed, and these will be given elsewhere.

\section{Violations of intrinsic ambipolarity due to unavoidable deviations from quasisymmetry}

We have seen that for a non-quasisymmetric stellarator the long-wavelength radial electric field is neoclassical, and therefore determined by equations (73) and (114). For a quasisymmetric stellarator the long-wavelength radial electric field is undetermined to this order, and the higher-order pieces given by equations (79) and (86) will be necessary to calculate it. Our final objective is to give in the future a model valid for both types of stellarators. Garren and Boozer proved in [4] that, except for the axisymmetric case, no toroidal quasisymmetric magnetic field exists. Therefore, it is important to understand how certain important quantities such as $\left\langle\boldsymbol{J} \cdot \nabla_{\boldsymbol{R}} \psi\right\rangle_{\psi}$ respond to the unavoidable deviation of the magnetic field from quasisymmetry.

Having finished our asymptotic expansions, we restore dimensionful variables. We find it appropriate for this section, and especially for the discussion in section 7.1. We omit the gyrophase because we only deal with gyrophase-independent functions. Then, we write the flux-surface average of the radial electric current as

$$
\left\langle\boldsymbol{J} \cdot \nabla_{\boldsymbol{R}} \psi\right\rangle_{\psi}=2 \pi\left\langle\sum_{\sigma} Z_{\sigma} e \int B \boldsymbol{v}_{M, \sigma} \cdot \nabla_{\boldsymbol{R}} \psi G_{\sigma 1} \mathrm{~d} u \mathrm{~d} \mu\right\rangle_{\psi},
$$

where we have simplified the notation by dropping the integration limits, the superindex $\mathrm{lw}$ on $G_{\sigma 1}$, and some arguments of the functions in the integrand. The function $G_{\sigma 1}$ is the non-adiabatic piece of the distribution function, $G_{\sigma 1}=F_{\sigma 1}+\left(Z_{\sigma} e \varphi_{1} / T_{\sigma}\right) F_{\sigma 0}$, and satisfies

$$
\begin{aligned}
(u \hat{\boldsymbol{b}} & \left.\cdot \nabla_{\boldsymbol{R}}-\mu \hat{\boldsymbol{b}} \cdot \nabla_{\boldsymbol{R}} B \partial_{u}\right) G_{\sigma 1} \\
& +\left(\frac{Z_{\sigma} e}{T_{\sigma}} \partial_{\psi} \varphi_{0}+\Upsilon_{\sigma}\right) \boldsymbol{v}_{M, \sigma} \cdot \nabla_{\boldsymbol{R}} \psi F_{\sigma 0}=C_{\sigma}^{\ell}\left[G_{1}\right],
\end{aligned}
$$

with $C_{\sigma}^{\ell}\left[G_{1}\right]$ the linearized collision operator,

$$
\begin{aligned}
& C_{\sigma}^{\ell}\left[G_{1}\right]:=\sum_{\sigma^{\prime}}\left(\mathcal{T}_{\sigma, 0}^{*} C_{\sigma \sigma^{\prime}}\left[\mathcal{T}_{\sigma, 0}^{*-1} G_{\sigma 1}, \mathcal{T}_{\sigma^{\prime}, 0}^{*-1} F_{\sigma^{\prime} 0}\right]\right. \\
& \left.+\mathcal{T}_{\sigma, 0}^{*} C_{\sigma \sigma^{\prime}}\left[\mathcal{T}_{\sigma, 0}^{*-1} F_{\sigma 0}, \mathcal{T}_{\sigma^{\prime}, 0}^{*-1} G_{\sigma^{\prime} 1}\right]\right) \\
& \Upsilon_{\sigma}:=\frac{1}{n_{\sigma}} \partial_{\psi} n_{\sigma}+\left(\frac{m_{\sigma}\left(u^{2} / 2+\mu B\right)}{T_{\sigma}}-\frac{3}{2}\right) \frac{1}{T_{\sigma}} \partial_{\psi} T_{\sigma},
\end{aligned}
$$


and

$F_{\sigma 0}(\boldsymbol{R}, u, \mu)=n_{\sigma}\left(\frac{m_{\sigma}}{2 \pi T_{\sigma}}\right)^{3 / 2} \exp \left(-\frac{m_{\sigma}\left(u^{2} / 2+\mu B\right)}{T_{\sigma}}\right)$.

Recall that $n_{\sigma}$ and $T_{\sigma}$ depend only on $\psi$ and that $T_{\sigma}=T_{\sigma^{\prime}}$ for every pair $\sigma, \sigma^{\prime}$. We stress that in dimensionful coordinates $F_{\sigma}=F_{\sigma 0}+F_{\sigma 1}+O\left(\epsilon_{\sigma}^{2} F_{\sigma 0}\right)$. Finally, the magnetic drift velocity can be written as

$$
\boldsymbol{v}_{M, \sigma}=\frac{1}{\Omega_{\sigma}} \hat{\boldsymbol{b}} \times\left(u^{2} \kappa+\mu \nabla_{\boldsymbol{R}} B\right) .
$$

Let us be more concrete about our aim. Assume that our magnetic field can be written as $\boldsymbol{B}=\boldsymbol{B}_{0}+\alpha \boldsymbol{B}_{1}$, where $\boldsymbol{B}_{0}$ is quasisymmetric and $\alpha$ is small. We want to expand (144) in powers of $\alpha$ and show that

$$
\left\langle\boldsymbol{J} \cdot \nabla_{\boldsymbol{R}} \psi\right\rangle_{\psi}=O\left(\alpha^{2}\right) .
$$

We will see that as soon as we employ the appropriate mathematical language, the proof will become very easy.

For an arbitrary stellarator magnetic field that satisfies $\left(\nabla_{\boldsymbol{R}} \times \boldsymbol{B}\right) \cdot \nabla_{\boldsymbol{R}} \psi \equiv 0$ (a magnetohydrodynamic equilibrium is a particular case), there exist Boozer coordinates [52], $\{\psi, \Theta, \zeta\}$, in which

$$
\boldsymbol{B}=-\tilde{\eta} \nabla_{\boldsymbol{R}} \psi+\frac{I(\psi)}{2 \pi} \nabla_{\boldsymbol{R}} \Theta+\frac{J(\psi)}{2 \pi} \nabla_{\boldsymbol{R}} \zeta
$$

and

$$
\boldsymbol{B}=\frac{\Psi_{\mathrm{p}}^{\prime}(\psi)}{2 \pi} \nabla_{\boldsymbol{R}} \zeta \times \nabla_{\boldsymbol{R}} \psi+\frac{\Psi_{\mathrm{t}}^{\prime}(\psi)}{2 \pi} \nabla_{\boldsymbol{R}} \psi \times \nabla_{\boldsymbol{R}} \Theta .
$$

Here, ' denotes differentiation with respect to $\psi, \Psi_{\mathrm{t}}$ is the toroidal flux, $\Psi_{\mathrm{p}}$ the poloidal flux, and $\tilde{\eta}(\psi, \Theta, \zeta)$ is a singlyvalued function. The metric determinant can be expressed in terms of the magnitude of the magnetic field,

$$
\sqrt{g}=\frac{V^{\prime}\left\langle B^{2}\right\rangle_{\psi}}{4 \pi^{2} B^{2}}
$$

and the derivative along the magnetic field reads

$$
\hat{\boldsymbol{b}} \cdot \nabla_{\boldsymbol{R}}=\frac{2 \pi \Psi_{\mathrm{t}}^{\prime} B}{\left\langle B^{2}\right\rangle_{\psi} V^{\prime}}\left(t \partial_{\Theta}+\partial_{\zeta}\right)
$$

where $t(\psi)=\Psi_{\mathrm{p}}^{\prime}(\psi) / \Psi_{\mathrm{t}}^{\prime}(\psi)$ is the rotational transform. As for the radial component of the magnetic drift,

$v_{\psi, \sigma}:=v_{M, \sigma} \cdot \nabla_{R} \psi=\frac{2 \pi m_{\sigma} c\left(u^{2}+\mu B\right)}{Z_{\sigma} e V^{\prime}\left\langle B^{2}\right\rangle_{\psi} B}\left(I \partial_{\zeta} B-J \partial_{\Theta} B\right)$,

where $\left(\nabla_{\boldsymbol{R}} \times \boldsymbol{B}\right) \cdot \nabla_{\boldsymbol{R}} \psi \equiv 0$ has been used. Therefore,

$$
\begin{aligned}
& \left\langle\boldsymbol{J} \cdot \nabla_{\boldsymbol{R}} \psi\right\rangle_{\psi}= \\
& \sum_{\sigma} \frac{m_{\sigma} c}{V^{\prime}} \oint \mathrm{d} \Theta \mathrm{d} \zeta \int \frac{u^{2}+\mu B}{B^{2}}\left(I \partial_{\zeta} B-J \partial_{\Theta} B\right) G_{\sigma 1} \mathrm{~d} u \mathrm{~d} \mu,
\end{aligned}
$$

where $\oint$ denotes an integral over $[0,2 \pi]$ in the angular variables. Equations (145), (154), (155), (156), and the fact that the kernel of the collision operator in drift-kinetic coordinates depends on the magnetic field only through $B$ (see appendix $\mathrm{G})$ means that the function $B(\psi, \Theta, \zeta)$ contains all the magnetic geometry information that is needed for the driftkinetic equation and for $\left\langle\boldsymbol{J} \cdot \nabla_{\boldsymbol{R}} \psi\right\rangle_{\psi}$. Put in different words, take two different stellarators and find Boozer coordinates for each of them. Then, formally, (145) and (156) differ for these devices, at most, in the function $B(\psi, \Theta, \zeta)$.

Now, we need to recall another way of characterizing quasisymmetric systems. In [1] it was shown that a magnetic field satisfies (140) if and only if, in Boozer coordinates $(\psi, \Theta, \zeta)$, the modulus of the magnetic field depends only on a single helicity, say $B \equiv B(\psi, M \Theta-N \zeta)$ for some pair $(M, N)$. Actually, equilibrium conditions near the magnetic axis enforce $M=1$ [15].

Thus, the result by Garren and Boozer [4] means that our stellarator magnetic field, once recast in Boozer coordinates, has a modulus that at best can be written as $B(\psi, \Theta, \zeta)=B_{0}(\psi, \Theta-N \zeta)+\alpha B_{1}(\psi, \Theta, \zeta)$, with small $\alpha$. The perturbation $B_{1}$ does not contain the helicity $\Theta-N \zeta$. Without loss of generality, we can take $B_{0}$ quasi-axisymmetric, $\partial_{\zeta} B_{0} \equiv 0$, which corresponds to $N=0^{4}$. Since $B_{1}$ does not contain the helicity of $B_{0}$, then

$$
\int B_{1}(\psi, \Theta, \zeta) \mathrm{d} \zeta=0
$$

We are ready to prove (150). The $O\left(\alpha^{0}\right)$ terms of (156) vanish due to quasisymmetry. The $O(\alpha)$ terms, $\left\langle\boldsymbol{J} \cdot \nabla_{\boldsymbol{R}} \psi\right\rangle_{\psi}^{(1)}$, are

$$
\begin{aligned}
\langle\boldsymbol{J} & \left.\cdot \nabla_{\boldsymbol{R}} \psi\right\rangle_{\psi}^{(1)} \\
= & -\sum_{\sigma} \frac{m_{\sigma} c}{V^{\prime}} \oint \mathrm{d} \Theta \mathrm{d} \zeta \int \frac{u^{2}+\mu B_{0}}{B_{0}^{2}} J \partial_{\Theta} B_{0} G_{\sigma}^{(1)} \mathrm{d} u \mathrm{~d} \mu \\
& +\sum_{\sigma} \frac{m_{\sigma} c}{V^{\prime}} \oint \mathrm{d} \Theta \mathrm{d} \zeta \int\left[\frac{2 u^{2}+\mu B_{0}}{B_{0}^{3}} B_{1} J \partial_{\Theta} B_{0}\right. \\
& \left.+\frac{u^{2}+\mu B_{0}}{B_{0}^{2}}\left(I \partial_{\zeta} B_{1}-J \partial_{\Theta} B_{1}\right)\right] G_{\sigma}^{(0)} \mathrm{d} u \mathrm{~d} \mu
\end{aligned}
$$

Here,

$$
G_{\sigma 1}:=G_{\sigma}^{(0)}+\alpha G_{\sigma}^{(1)}+O\left(\alpha^{2}\right)
$$

where the coefficients are determined by the equations

$$
\begin{aligned}
& \left(u \hat{\boldsymbol{b}} \cdot \nabla_{\boldsymbol{R}}-\mu \hat{\boldsymbol{b}} \cdot \nabla_{\boldsymbol{R}} B \partial_{u}\right)^{(0)} G_{\sigma}^{(0)} \\
& \quad+\left(\frac{Z_{\sigma} e}{T_{\sigma}} \partial_{\psi} \varphi_{0}+\Upsilon_{\sigma}\right)\left(\boldsymbol{v}_{M, \sigma} \cdot \nabla_{\boldsymbol{R}} \psi\right)^{(0)} F_{\sigma 0}^{(0)} \\
& =C_{\sigma}^{\ell(0)}\left[G^{(0)}\right],
\end{aligned}
$$

4 If $B$ is helically symmetric, $N \neq 0$, then one can define $\bar{\Theta}:=\Theta-N \zeta$. The coordinates $\bar{\Theta}, \zeta$ are Boozer angles and in terms of them the problem reduces to the quasi-axisymmetric case. 


$$
\begin{aligned}
(u \hat{\boldsymbol{b}} & \left.\cdot \nabla_{\boldsymbol{R}}-\mu \hat{\boldsymbol{b}} \cdot \nabla_{\boldsymbol{R}} B \partial_{u}\right)^{(0)} G_{\sigma}^{(1)} \\
& +\left(u \hat{\boldsymbol{b}} \cdot \nabla_{\boldsymbol{R}}-\mu \hat{\boldsymbol{b}} \cdot \nabla_{\boldsymbol{R}} B \partial_{u}\right)^{(1)} G_{\sigma}^{(0)} \\
& +\left[\left(\frac{Z_{\sigma} e}{T_{\sigma}} \partial_{\psi} \varphi_{0}+\Upsilon_{\sigma}\right)\left(\boldsymbol{v}_{M, \sigma} \cdot \nabla_{\boldsymbol{R}} \psi F_{\sigma 0}\right)\right]^{(1)} \\
= & C_{\sigma}^{\ell(1)}\left[G^{(0)}\right]+C_{\sigma}^{\ell(0)}\left[G^{(1)}\right] .
\end{aligned}
$$

Obviously,

$$
\begin{aligned}
& \left(\hat{\boldsymbol{b}} \cdot \nabla_{\boldsymbol{R}}\right)^{(0)}=\frac{2 \pi \Psi_{\mathrm{t}}^{\prime} B_{0}}{\left\langle B^{2}\right\rangle_{\psi} V^{\prime}}\left(t \partial_{\Theta}+\partial_{\zeta}\right), \\
& \left(\hat{\boldsymbol{b}} \cdot \nabla_{\boldsymbol{R}}\right)^{(1)}=\frac{2 \pi \Psi_{\mathrm{t}}^{\prime} B_{1}}{\left\langle B^{2}\right\rangle_{\psi} V^{\prime}}\left(t \partial_{\Theta}+\partial_{\zeta}\right), \\
& \left(\hat{\boldsymbol{b}} \cdot \nabla_{\boldsymbol{R}} B\right)^{(0)}=\frac{2 \pi \Psi_{\mathrm{t}}^{\prime} B_{0}}{\left\langle B^{2}\right\rangle_{\psi} V^{\prime}} t \partial_{\Theta} B_{0}, \\
& \left(\hat{\boldsymbol{b}} \cdot \nabla_{\boldsymbol{R}} B\right)^{(1)}=\frac{2 \pi \Psi_{\mathrm{t}}^{\prime}}{\left\langle B^{2}\right\rangle_{\psi} V^{\prime}}\left[B_{1} t \partial_{\Theta} B_{0}+B_{0}\left(t \partial_{\Theta}+\partial_{\zeta}\right) B_{1}\right],
\end{aligned}
$$

etc. In appendix $\mathrm{G}$ we have computed explicitly the form of the Fokker-Planck collision operator in coordinates $(\boldsymbol{R}, u, \mu)=$ $\mathcal{T}_{\sigma, 0}^{-1}(\boldsymbol{r}, \boldsymbol{v})$, and we make it clear that the kernel depends on the magnetic geometry only via the magnitude of $\boldsymbol{B}$. We will not give fully explicit expressions for the pieces of the linearized operator, $C_{\sigma}^{\ell(1)}\left[G^{(0)}\right]$ and $C_{\sigma}^{\ell(0)}\left[G^{(1)}\right]$, because they are cumbersome and we will not exploit them at all. Equations (G.14), (G.15), and (G.16) are enough to realize that $C_{\sigma}^{\ell(1)}\left[G^{(0)}\right]$ is linear in $B_{1}$ and that the kernel defining the piece $C_{\sigma}^{\ell(0)}\left[G^{(1)}\right]$ does not depend on $\zeta$. Finally, from (160) and (161), one immediately notes that

$$
\partial_{\zeta} G_{\sigma}^{(0)}=0
$$

and

$$
\int_{0}^{2 \pi} G_{\sigma}^{(1)} \mathrm{d} \zeta=0
$$

Hence, we infer that every term on the right-hand side of (158) is of the form

$$
\oint f(\psi, \Theta) g(\psi, \Theta, \zeta) \mathrm{d} \Theta \mathrm{d} \zeta
$$

where

$$
\int_{0}^{2 \pi} g(\psi, \Theta, \zeta) \mathrm{d} \zeta=0
$$

Then, the result $\left\langle\boldsymbol{J} \cdot \nabla_{\boldsymbol{R}} \psi\right\rangle_{\psi}^{(1)} \equiv 0$ follows. The terms $O\left(\alpha^{2}\right)$ do not vanish, in general, so $\left\langle\boldsymbol{J} \cdot \nabla_{\boldsymbol{R}} \psi\right\rangle_{\psi}=O\left(\alpha^{2}\right)$ as far as the lowest-order neoclassical terms are concerned. This result was obtained in a short mean-free path plasma, employing fluid equations, in [5]. Unsurprisingly, flow damping due to small magnetic ripple in tokamaks also follows this scaling (see, for example, [53]).

Therefore, for small deviations from quasisymmetry, the flux-surface-averaged radial current can be written as

$$
\begin{aligned}
\langle\boldsymbol{J} \cdot & \left.\nabla_{\boldsymbol{R}} \psi\right\rangle_{\psi}=\left(\epsilon_{\mathrm{s}}^{2} \alpha^{2} A+\epsilon_{\mathrm{s}}^{3} C\right) e n_{\mathrm{e} 0} c_{\mathrm{s}}\left|\nabla_{\boldsymbol{R}} \psi\right|_{0} \\
& +O\left(\epsilon_{\mathrm{s}}^{4} e n_{\mathrm{e} 0} c_{\mathrm{s}}\left|\nabla_{\boldsymbol{R}} \psi\right|_{0}\right)
\end{aligned}
$$

where $A$ and $C$ are $O(1)$, and $\left|\nabla_{R} \psi\right|_{0}$ is a characteristic value of $\left|\nabla_{\boldsymbol{R}} \psi\right|$. The coefficient $A$ is determined from neoclassical theory, whereas $C$ has neoclassical and turbulent contributions. Note that the dependence of (170) on $\alpha$ has been inferred in this section and the dependence on $\epsilon_{\mathrm{s}}$ is an immediate consequence of the results of previous ones: the fact that the lowest-order neoclassical contribution is $O\left(\epsilon_{\mathrm{s}}^{2}\right)$ may be understood from inspection of (144). In addition, when we derived the lowestorder flux-surface-averaged radial current, we deduced that turbulence does not contribute to it; that is, we proved that to $O\left(\epsilon_{\mathrm{s}}^{2}\right)$ the quantity $\left\langle\boldsymbol{J} \cdot \nabla_{\boldsymbol{R}} \psi\right\rangle_{\psi}$ is exactly given by the righthand side of (144). Hence, turbulent contributions can only enter to $O\left(\epsilon_{\mathrm{s}}^{3}\right)$ or higher.

It is clear that if $\alpha>\epsilon_{\mathrm{s}}^{1 / 2}$, then the long-wavelength radial electric field is set neoclassically. Finally, if the magnetic field possesses stellarator symmetry, $\epsilon_{\mathrm{s}}^{3} C$ in (170) has to be replaced by $\epsilon_{\mathrm{s}}^{4} C$ [54], and therefore $\varphi_{0}$ is neoclassical when $\alpha>\epsilon_{\mathrm{s}}$.

The criterion for rotation, however, does not depend on the existence or not of stellarator symmetry: the stellarator is able to rotate to high speeds if $\alpha<\epsilon_{\mathrm{s}}^{1 / 2}$. Consider the total momentum conservation equation,

$$
\begin{aligned}
\partial_{t}\left(n_{\mathrm{i}} m_{\mathrm{i}} \boldsymbol{V}_{\mathrm{i}}\right) & =-\nabla_{r} \cdot\left[\stackrel{\leftrightarrow}{\Pi}_{i}+p_{\perp}(\stackrel{\leftrightarrow}{\boldsymbol{I}}-\hat{\boldsymbol{b}} \hat{\boldsymbol{b}})+p_{\|} \hat{\boldsymbol{b}} \hat{\boldsymbol{b}}\right] \\
& +\frac{1}{c} \boldsymbol{J} \times \boldsymbol{B},
\end{aligned}
$$

where $V_{\mathrm{i}}$ is the ion flow, $p_{\perp}$ and $p_{\|}$are the total perpendicular and parallel pressures, and $\stackrel{\leftrightarrow}{\Pi}_{i}$ contains the turbulent contributions to the ion stress tensor. Electron inertial terms and turbulent terms corresponding to electrons are small in a $\sqrt{m_{\mathrm{e}} / m_{\mathrm{i}}} \ll 1$ expansion and have been neglected. As in [1], we will employ a solenoidal vector field $S$ of the form

$$
\boldsymbol{S}=-\frac{c}{B} \hat{\boldsymbol{b}} \times \nabla_{r} \psi+\xi \boldsymbol{B} .
$$

The function $\xi(\psi, \Theta, \zeta)$ is determined by imposing $\nabla_{r} \cdot S=0$. This equation can be solved if $\left(\nabla_{r} \times \boldsymbol{B}\right) \cdot \nabla_{r} \psi \equiv 0$. Taking the scalar product of (171) with $\boldsymbol{S}$ gives, in steady state,

$$
\left\langle\boldsymbol{J} \cdot \nabla_{\boldsymbol{R}} \psi\right\rangle_{\psi}=\Lambda^{\mathrm{neo}}+\Lambda^{\mathrm{tb}},
$$

where

$$
\Lambda^{\text {neo }}=\left\langle\left(p_{\|}-p_{\perp}\right)(\stackrel{\leftrightarrow}{\boldsymbol{I}} / 3-\hat{\boldsymbol{b}} \hat{\boldsymbol{b}}): \nabla_{r} S\right\rangle_{\psi}
$$

and

$$
\Lambda^{\mathrm{tb}}=\frac{1}{V^{\prime}} \partial_{\psi}\left\langle V^{\prime} \boldsymbol{S} \cdot \stackrel{\leftrightarrow}{\boldsymbol{\Pi}}_{i} \cdot \nabla_{r} \psi\right\rangle_{\psi}-\left\langle\stackrel{\leftrightarrow}{\boldsymbol{\Pi}}_{i}: \nabla_{r} S\right\rangle_{\psi}
$$

If $\alpha$ is sufficiently large, $\left\langle\boldsymbol{J} \cdot \nabla_{\boldsymbol{R}} \psi\right\rangle_{\psi} \sim \Lambda^{\text {neo }}$ and $\Lambda^{\text {tb }}$ can be neglected. Then, the radial electric field is set by the neoclassical ambipolarity condition and the resulting flow is subsonic. Hence, in order to have sonic flows we need $\left\langle\boldsymbol{J} \cdot \nabla_{\boldsymbol{R}} \psi\right\rangle_{\psi} \sim \Lambda^{\mathrm{tb}}$. Recall the transport time scale $\tau_{E}=$ $\epsilon_{\mathrm{s}}^{-2} L / c_{\mathrm{s}}$ defined in section 2.1. Assuming that the transport of momentum satisfies

$$
\Lambda^{\mathrm{tb}} \sim \frac{c n_{\mathrm{i}} m_{\mathrm{i}} V_{\mathrm{i}}\left|\nabla_{r} \psi\right|}{\tau_{E} B},
$$




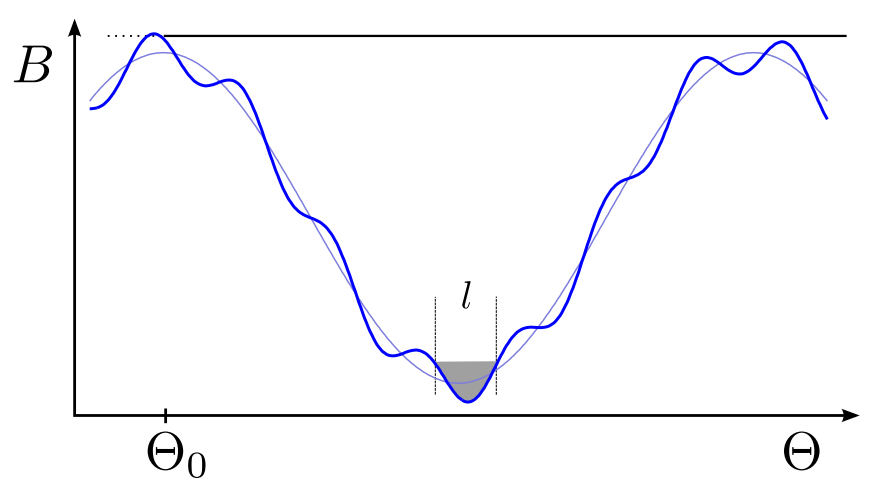

Figure 1. The dependence of the magnitude of the magnetic field on the distance along the field line in a quasisymmetric configuration (thin curve), and the result after a perturbation that makes it non-quasisymmetric has been added (thick curve). Trapped particles in a magnetic well of size $l$ created by the perturbation are confined to the shaded region. The trajectory of a particle that was passing and becomes barely trapped after including the perturbation is also represented.

we have that $V_{\mathrm{i}} \sim v_{\mathrm{ti}}$ requires $\left\langle\boldsymbol{J} \cdot \nabla_{\boldsymbol{R}} \psi\right\rangle_{\psi} \sim \epsilon_{\mathrm{s}}^{3} e n_{\mathrm{e} 0} c_{\mathrm{s}}\left|\nabla_{\boldsymbol{r}} \psi\right|_{0}$. Since

$$
\Lambda^{\text {neo }} \sim \epsilon_{\mathrm{s}}^{2} \alpha^{2} e n_{\mathrm{e} 0} c_{\mathrm{s}}\left|\nabla_{r} \psi\right|_{0},
$$

we need $\alpha<\epsilon_{\mathrm{s}}^{1 / 2}$. It is true that $\Lambda^{\mathrm{tb}} \sim \epsilon_{\mathrm{s}}^{4} e n_{\mathrm{e} 0} c_{\mathrm{s}}\left|\nabla_{R} \psi\right|_{0}$ in a stellarator symmetric device, and the consequence of this is that the intrinsic rotation will be subsonic. To get sonic rotation, we need external sources of momentum such as neutral beams or radio frequency momentum injection.

\subsection{Breakdown of the expansion}

One might expect that our expansion in integer powers of $\alpha$, and hence the scaling of the radial current with $\alpha^{2}$, break down in certain circumstances (even if $\alpha \ll 1$ ) because of the usual notion that helically trapped particles give transport that scales with a fractional power of the depth of the magnetic wells (see, for example, [1]). In what follows, we discuss the validity of the expansion in integer powers of $\alpha$.

In equation (145), consider the factor $\hat{\boldsymbol{b}} \cdot \nabla_{\boldsymbol{R}} B$ in the parallel streaming operator. As long as $\alpha\left(\hat{b} \cdot \nabla_{R}\right)^{(0)} B_{1} \ll$ $\left(\hat{\boldsymbol{b}} \cdot \nabla_{\boldsymbol{R}}\right)^{(0)} B_{0}$, our expansion can be carried out for any value of the collisionality. However, if

$$
\alpha\left(\hat{\boldsymbol{b}} \cdot \nabla_{\boldsymbol{R}}\right)^{(0)} B_{1} \sim\left(\hat{\boldsymbol{b}} \cdot \nabla_{\boldsymbol{R}}\right)^{(0)} B_{0}
$$

somewhere, then we cannot guarantee that the expansion correctly describes the behavior of particles in the whole phase space. The gradients in (178) can be comparable in two qualitatively different situations that we treat separately (see figure 1). First, equation (178) holds near points where $\left(\hat{\boldsymbol{b}} \cdot \nabla_{\boldsymbol{R}}\right)^{(0)} B_{0}=0$, and this is related to almost trapped, barely trapped, and deeply trapped particles in the magnetic field $B_{0}$. There always exist points like these ones, and we deal with them later on.

Second, in generic points where $\left(\hat{\boldsymbol{b}} \cdot \nabla_{\boldsymbol{R}}\right)^{(0)} B_{0} \sim B_{0} L^{-1}$, condition (178) is met if and only if

$$
\frac{l}{L} \sim \alpha,
$$
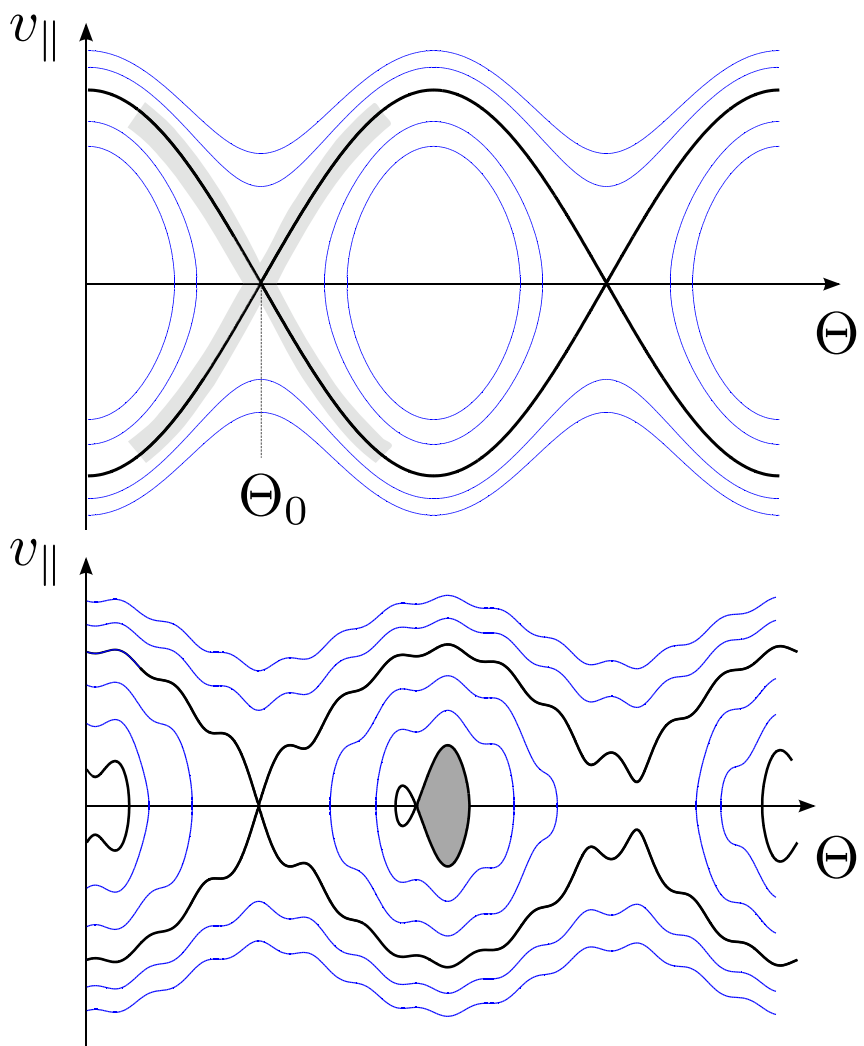

Figure 2. Some contours of constant kinetic energy for the quasisymmetric magnetic field (top) and perturbed magnetic field (bottom) of figure 1.

where $L$ is the characteristic length of variation of $B_{0}$ and $l$ that of $B_{1}$. Then, the perturbation $\alpha B_{1}$ can create new magnetic wells of typical width $l$. Given $\alpha$, (179) is satisfied only if the helicity of the perturbation $B_{1}$ is large enough. Particles trapped in the new helical wells may seem the main contribution to the breaking of quasisymmetry (see [1]), but in most cases the large gradients $\alpha \nabla_{R} B_{1} \sim \nabla_{R} B_{0}$ will not be exclusively parallel to the magnetic field. Typically, $\nabla_{R} B_{1}$ will modify $v_{\psi, \sigma}$ enough to make $\alpha v_{\psi, \sigma}^{(1)} \sim v_{\psi, \sigma}^{(0)}$. The problem is that, if this is the case, one cannot derive a scaling with $\alpha$ for the distribution function outside the wells. Consequently, one cannot find out whether helically trapped particles dominate transport, and no scaling for the radial electric current can be derived either. As a result, our expansion is only valid for

$$
\frac{l}{L} \gg \alpha
$$

If large helicity perturbations are unavoidable, then to get close to quasisymmetry the design should make the large gradients $\alpha \nabla_{R} B_{1}$ aligned in such a way that $\alpha v_{\psi, \sigma}^{(1)} \ll v_{\psi, \sigma}^{(0)}$. Let us assume that (180) is satisfied and hence no new magnetic wells are created. Then, $\alpha\left(\hat{\boldsymbol{b}} \cdot \nabla_{\boldsymbol{R}}\right)^{(0)} B_{1} \sim(\hat{\boldsymbol{b}}$. $\left.\nabla_{R}\right)^{(0)} B_{0}$ can only be satisfied in the vicinity of points where $\left(\hat{\boldsymbol{b}} \cdot \nabla_{\boldsymbol{R}}\right)^{(0)} B_{0}=0$; points like $\Theta_{0}$ in figures 1 and 2 . The trajectories that may qualitatively change with the perturbation $\alpha B_{1}$ are the ones close to the black, thick line in figure 2 (top). Those are the trajectories of particles that are almost trapped or barely trapped in $B_{0}$. For example, as is clearly seen in figure 1 
and in figure 2 (bottom), a particle that was passing in the quasisymmetric field can become trapped in a large magnetic well because $B=B_{0}+\alpha B_{1}$ at $\Theta_{0}$ is larger than $B_{0}$. These particles are not necessarily trapped in the wells created by $B_{0}$, and in general will be trapped in large wells that extend several of the wells due to $B_{0}$. The trajectory shown in figure 1 , for example, spans at least two of the wells created by $B_{0}$. We proceed to find the scaling of the radial current due to these barely trapped or almost trapped particles.

First, let us determine the size (in parallel velocity) of the region of phase space that we are interested in. In the vicinity of $\Theta_{0}$,

$$
B_{0}=B_{0}\left(\Theta_{0}\right)+\frac{1}{2} \partial_{\Theta}^{2} B_{0}\left(\Theta_{0}\right)\left(\Theta-\Theta_{0}\right)^{2} .
$$

After adding the perturbation $\alpha B_{1}$ we get, in that region,

$$
\begin{aligned}
B= & B_{0}\left(\Theta_{0}\right)+\frac{1}{2} \partial_{\Theta}^{2} B_{0}\left(\Theta_{0}\right)\left(\Theta-\Theta_{0}\right)^{2} \\
& +\alpha B_{1}\left(\Theta_{0}\right)+\alpha \partial_{\Theta} B_{1}\left(\Theta_{0}\right)\left(\Theta-\Theta_{0}\right) \\
= & B_{0}\left(\Theta_{0}\right)+\frac{1}{2} \partial_{\Theta}^{2} B_{0}\left(\Theta_{0}\right)\left(\Theta-\Theta_{0}+\alpha \frac{\partial_{\Theta} B_{1}\left(\Theta_{0}\right)}{\partial_{\Theta}^{2} B_{0}\left(\Theta_{0}\right)}\right)^{2} \\
& +\alpha B_{1}\left(\Theta_{0}\right)+O\left(\alpha^{2}\right) .
\end{aligned}
$$

The perturbation $\alpha B_{1}$ moves the maximum of $B$ a distance $O(\alpha)$ in $\Theta$ and it modifies the maximum value of $B, B_{0}\left(\Theta_{0}\right)$, by a quantity of $O(\alpha)$. Denote by $v_{\|}$the parallel velocity viewed as a function of the kinetic energy $\varepsilon=u^{2} / 2+\mu B(\boldsymbol{R})$, the magnetic moment $\mu$, and the position in space $R, v_{\|}=$ $\sqrt{2(\varepsilon-\mu B(R))}$. Due to these changes, the parallel velocity $v_{\|}$of a barely trapped or almost trapped particle differs by a quantity of $O\left(v_{\|}\right)$from the velocity $v_{\| 0}$ obtained at the same location $\Theta$ for the quasisymmetric magnetic field $B_{0}$. The expansion for small $\alpha$ in (160) and (161) does not work when the perturbation to the parallel velocity is not small in $\alpha$. We can estimate the number of these particles and which part of their trajectory is significantly modified. In the vicinity of $\Theta_{0}$,

$$
\begin{aligned}
v_{\|}= & {\left[v_{\| 0}^{2}-2 \mu \alpha B_{1}\left(\Theta_{0}\right)\right.} \\
& \left.-\frac{\mu}{2} \partial_{\Theta}^{2} B_{0}\left(\Theta_{0}\right)\left(\Theta-\Theta_{0}+\alpha \frac{\partial_{\Theta} B_{1}\left(\Theta_{0}\right)}{\partial_{\Theta}^{2} B_{0}\left(\Theta_{0}\right)}\right)^{2}\right]^{1 / 2},
\end{aligned}
$$

where $v_{\| 0}=\sqrt{2\left(\varepsilon-\mu B_{0}\left(\Theta_{0}\right)\right)}$. It is clear that the perturbation $\alpha B_{1}$ will significantly modify the parallel velocity of particles with $v_{||} \sim \sqrt{\alpha} c_{\mathrm{s}}$ in a vicinity $\left|\Theta-\Theta_{0}\right| \sim \sqrt{\alpha}$ of $\Theta_{0}$. In figure 2 (top), the region in phase space that these particles occupy is a band with thickness $\Delta v_{\|} / c_{\mathrm{s}} \sim \sqrt{\alpha}$ around the orbit plotted with a black, thick line. Only in the part of that band that satisfies $\left|\Theta-\Theta_{0}\right| \sim \sqrt{\alpha}$ is the orbit significantly modified by the perturbation $\alpha B_{1}$. This part is lightly shaded in figure 2 (top).

Now, we are ready to derive the scaling of the distribution function and, then, the scaling of the radial electric current. The relevant collisionality regime is the $1 / v$ regime. In highly collisional plasmas, it was proven in [5], with fluid equations, that an $\alpha^{2}$ scaling holds. Let $v_{\sigma}$ be the maximum of the collision frequencies $v_{\sigma \sigma^{\prime}}$ when $\sigma^{\prime}$ runs over all the species, and $v_{* \sigma}=v_{\sigma} L / c_{\mathrm{s}}$ the corresponding collisionality. In the $1 / v$ regime the parallel streaming terms of (145) are the largest ones. Define an expansion of $G_{\sigma 1}$ in $\nu_{* \sigma} \ll 1$ by

$$
G_{\sigma 1}=G_{\sigma}^{[-1]}+G_{\sigma}^{[0]}+\cdots,
$$

where $G_{\sigma}^{[j]}=O\left(v_{* \sigma}^{j} \epsilon_{\sigma} F_{\sigma 0}\right)$. To lowest order in this small collisionality expansion, equation (145) reads

$$
\left(u \hat{\boldsymbol{b}} \cdot \nabla_{\boldsymbol{R}}-\mu \hat{\boldsymbol{b}} \cdot \nabla_{\boldsymbol{R}} B \partial_{u}\right) G_{\sigma}^{[-1]}=0,
$$

so $G_{\sigma}^{[-1]}$ is constant over orbits, $\overline{G_{\sigma}^{[-1]}}=G_{\sigma}^{[-1]}$. Here,

$\overline{h(\psi, \Theta, \zeta, \varepsilon, \mu)}$

$$
=\frac{\int h(\psi, \Theta(l), \zeta(l), \varepsilon, \mu) v_{\|}^{-1}(\psi, \Theta(l), \zeta(l), \varepsilon, \mu) \mathrm{d} l}{\int v_{\|}^{-1}(\psi, \Theta(l), \zeta(l), \varepsilon, \mu) \mathrm{d} l}
$$

is the transit average of the function $h(\psi, \Theta, \zeta, \varepsilon, \mu)$, which is a time average over the particle trajectory. To lowest order the trajectory is the magnetic field line, $(\psi, \Theta(l), \zeta(l))$, with $l$ the arc length. The transit average is taken holding the kinetic energy $\varepsilon$ and the magnetic moment $\mu$ fixed. An order higher in $v_{* \sigma}$ than (185), the transit average of the Fokker-Planck equation (145) gives

$$
\left(\frac{Z_{\sigma} e}{T_{\sigma}} \partial_{\psi} \varphi_{0}+\Upsilon_{\sigma}\right) \overline{v_{\psi, \sigma}} F_{\sigma 0}=\overline{C_{\sigma}^{\ell}\left[G^{[-1]}\right]} .
$$

Clearly,

$$
G_{\sigma}^{[-1]} \sim \frac{1}{v_{\mathrm{eff}} B_{0} L^{2}} \overline{v_{\psi, \sigma}} F_{\sigma 0},
$$

where $v_{\text {eff }}:=v_{\sigma} / \alpha$ is the effective collision frequency. This effective collision frequency takes into account that the region of phase space where the perturbation $\alpha B_{1}$ modifies the trajectories significantly is a band with thickness $\Delta v_{\|} / c_{\mathrm{s}} \sim$ $\sqrt{\alpha}$, and it takes very few collisions $(\sim \alpha)$ for particles to diffuse out of this region into the part of phase space in which the trajectory is only modified by a correction of $O(\alpha)$. To obtain a final estimate for $G_{\sigma}^{[-1]}$, we need to find a bound for $\overline{v_{\psi, \sigma}}$. Away from the region $\left|\Theta-\Theta_{0}\right| \sim \sqrt{\alpha}$ where the orbits are modified significantly, the expansion in equations (160) and (161) is valid. When $\left|\Theta-\Theta_{0}\right| \sim \sqrt{\alpha}$, the magnetic drift is composed of the piece due to $B_{0}, v_{\psi, \sigma}^{(0)}$, and the piece due to $\alpha B_{1}, \alpha v_{\psi, \sigma}^{(1)}$. For the quasisymmetric magnetic field, the radial magnetic drift has the form

$$
v_{\psi, \sigma}^{(0)}=\frac{m_{\sigma} c}{Z_{\sigma} e}\left(u \hat{\boldsymbol{b}} \cdot \nabla_{\boldsymbol{R}}-\mu \hat{\boldsymbol{b}} \cdot \nabla_{\boldsymbol{R}} B \partial_{u}\right)^{(0)}\left(\chi(\psi) \frac{u}{B_{0}}\right) .
$$

Thus, $v_{\psi, \sigma}^{(0)}$ is zero at $\Theta_{0}$, where $\left(\hat{\boldsymbol{b}} \cdot \nabla_{\boldsymbol{R}}\right)^{(0)} B_{0}$, and it satisfies $v_{\psi, \sigma}^{(0)} \sim\left(\Theta-\Theta_{0}\right) \rho_{\mathrm{i}} c_{\mathrm{s}} B_{0}$ around $\Theta=\Theta_{0}$. In the region of interest, $\left|\Theta-\Theta_{0}\right| \sim \sqrt{\alpha}$, giving $v_{\psi, \sigma}^{(0)} \sim \sqrt{\alpha} \rho_{\mathrm{i}} c_{\mathrm{s}} B_{0} \gg \alpha v_{\psi, \sigma}^{(1)}$. We need to find how much this $v_{\psi, \sigma}^{(0)}$ contributes to the average $\overline{v_{\psi, \sigma}}$. The particle spends a time interval of $O\left(L / c_{\mathrm{s}}\right)$ in the region $\left|\Theta-\Theta_{0}\right| \gg \sqrt{\alpha}$, and $O\left(\sqrt{\alpha} L / \sqrt{\alpha} c_{\mathrm{s}}\right)=O\left(L / c_{\mathrm{s}}\right)$ in the region $\left|\Theta-\Theta_{0}\right| \sim \sqrt{\alpha}$, where the trajectory has a length of only $\sqrt{\alpha} L$ and a parallel velocity $v_{\|} \sim \sqrt{\alpha} c_{\mathrm{s}}$. Thus, 
the time spent in the much smaller region $\left|\Theta-\Theta_{0}\right| \sim \sqrt{\alpha}$ is equivalent to the time spent in the rest of the trajectory. Since the expansion in equations (160) and (161) works for $\left|\Theta-\Theta_{0}\right| \gg \sqrt{\alpha}$, giving a contribution of $O(\alpha)$ to $\overline{v_{\psi, \sigma}}$ (only the vanishing contribution of the quasisymmetric magnetic field enters to $\left.O\left(\alpha^{0}\right)\right), \overline{v_{\psi, \sigma}}$ is dominated by the region $\mid \Theta-$ $\Theta_{0} \mid \sim \sqrt{\alpha}$, giving $\overline{v_{\psi, \sigma}} \sim \sqrt{\alpha} \rho_{\mathrm{i}} c_{\mathrm{s}} B_{0}$. Then, $G_{\sigma}^{[-1]} \propto \alpha^{3 / 2}$ in the phase-space region where the expansion in (160) and (161) breaks. By employing (144), one gets the scaling of the contribution of these particles to the radial current, given by $e \sqrt{\alpha} c_{\mathrm{s}}^{3} G_{\sigma}^{[-1]} \overline{v_{\psi, \sigma}} \propto \alpha^{5 / 2}$, that is subdominant with respect to the $\alpha^{2}$ scaling. Thus, these particles do not dominate transport, and the scaling $\left\langle\boldsymbol{J} \cdot \nabla_{\boldsymbol{R}} \psi\right\rangle_{\psi} \sim \alpha^{2}$ will be observed.

In addition to barely trapped and almost trapped particles, deeply trapped particles can also be affected by perturbations that satisfy (180) because they move in a region close to a point with $\left(\hat{\boldsymbol{b}} \cdot \nabla_{\boldsymbol{R}}\right)^{(0)} B_{0}=0$ (see the grey wells in figures 1 and 2). Even if new wells are created in this region, and the trajectories of deeply trapped particles are modified, their contribution to the radial electric current scales also as $\alpha^{5 / 2}$ and hence is negligible.

\section{Conclusions}

Quasisymmetric stellarators can rotate freely in the symmetry direction whereas non-quasisymmetric ones cannot. In the latter, parallel viscosity damps the flow and sets the long-wavelength radial electric field. In the former, the component of the flow in the symmetry direction remains neoclassically undamped and the long-wavelength radial electric field undetermined in calculations to lowest-order in $\epsilon$, the Larmor radius over the characteristic macroscopic scale. One of the objectives of this paper is to take the first step towards a unified treatment of the long-wavelength radial electric field and the rotation that is valid for quasisymmetric and non-quasisymmetric stellarators. We have derived the gyrokinetic Fokker-Planck and quasineutrality equations to $O\left(\epsilon^{2}\right)$ for an arbitrary stellarator. Then, we have taken their long-wavelength limit. To $O(\epsilon)$ one gets the well-known drift-kinetic equation (73) and the first-order quasineutrality equation (85). By themselves, these first-order equations do not give the long-wavelength radial electric field. In a generic stellarator one expects to be able to solve for it by adding the neoclassical ambipolarity condition. How does it show up in the typical framework of gyrokinetic theory, i.e. a hierarchy of Fokker-Planck and quasineutrality equations derived order by order in $\epsilon$ ? It comes from the solvability conditions of the $O\left(\epsilon^{2}\right)$ piece of the Fokker-Planck equation, as explained in section 5.4. Then, one can ask whether the Fokker-Planck and quasineutrality equations to $O(\epsilon)$, together with the neoclassical ambipolarity condition, determine the long-wavelength radial electric field. The answer is affirmative if and only if the stellarator is not quasisymmetric.

When the stellarator is quasisymmetric, the neoclassical radial current does not give an equation for the longwavelength radial electric field (or equivalently, for the rotation along the symmetry direction) and the full gyrokinetic computation to order $O\left(\epsilon^{2}\right)$ is necessary. In particular, the long-wavelength equations derived here must be implemented in a code to obtain the second-order correction $\left\langle F_{\sigma 2}^{\mathrm{lw}}\right\rangle$ (see (79)). This piece of the distribution function will eventually enter the equation for the long-wavelength radial electric field obtained from higher-order solvability conditions. Equivalently, this piece is necessary to get the correct radial flux of momentum in a quasisymmetric stellarator. This is the reason that we have worked out all the expressions to second order explicitly. In order to have a complete set of equations that make it possible to obtain the long-wavelength radial electric field, the shortwavelength gyrokinetic equations to $O\left(\epsilon^{2}\right)$ must be derived. This will be done elsewhere.

As mentioned above, a stellarator is able to sustain large rotation, beneficial for confinement, if and only if it is quasisymmetric [1]. Since in [4] it was proven that, apart from the axisymmetric case, quasisymmetric stellarators do not exist, it is pertinent to ask when a stellarator can be considered quasisymmetric in practice. All the above results allow us to pose this question in precise terms. It is the absence of intrinsic ambipolarity that makes the computation of the radial electric field radically different, and prevents the stellarator from freely rotating, so it seems relevant to investigate how a sufficiently small deviation $\alpha \boldsymbol{B}_{1}$ from a quasisymmetric magnetic field $\boldsymbol{B}_{0}$ translates into a violation of intrinsic ambipolarity. We have found that, generally, the stellarator can be considered quasisymmetric in practice if

$$
\alpha<\epsilon^{1 / 2} \text {. }
$$

\section{Acknowledgments}

We acknowledge Matt Landreman for very interesting discussions and for suggesting some modifications to the first version of this paper. This research was supported in part by grant ENE2012-30832, Ministerio de Economía y Competitividad, and by US DoE grant DE-SC008435.

\section{Appendix A. Some basic properties of the collision operator}

We recall (see, for example, [55]) that the collision operator (4) satisfies, for every $\sigma, \sigma^{\prime}$, the particle number, momentum, and energy conservation properties

$$
\begin{gathered}
\int C_{\sigma \sigma^{\prime}} \mathrm{d}^{3} v=0, \\
\int m_{\sigma} \boldsymbol{v} C_{\sigma \sigma^{\prime}} \mathrm{d}^{3} v=-\int m_{\sigma^{\prime}} \boldsymbol{v} C_{\sigma^{\prime} \sigma} \mathrm{d}^{3} v, \\
\int \frac{1}{2} m_{\sigma} v^{2} C_{\sigma \sigma^{\prime}} \mathrm{d}^{3} v=-\int \frac{1}{2} m_{\sigma^{\prime}} \boldsymbol{v}^{2} C_{\sigma^{\prime} \sigma} \mathrm{d}^{3} v .
\end{gathered}
$$

The entropy production term is non-negative. That is,

$$
s=-\sum_{\sigma, \sigma^{\prime}} \int \ln f_{\sigma} C_{\sigma \sigma^{\prime}}\left[f_{\sigma}, f_{\sigma^{\prime}}\right] \mathrm{d}^{3} v
$$

always satisfies $s \geqslant 0$, and $s=0$ if and only if all of the distribution functions are Maxwellians with the same 
temperature and flow; i.e. $f_{\sigma}=f_{M \sigma}$, where

$$
\begin{gathered}
f_{M \sigma}(\boldsymbol{r}, \boldsymbol{v})=n_{\sigma}(\boldsymbol{r})\left(\frac{m_{\sigma}}{2 \pi T_{\sigma}(\boldsymbol{r})}\right)^{3 / 2} \\
\times \exp \left(-\frac{m_{\sigma}(\boldsymbol{v}-\boldsymbol{V}(\boldsymbol{r}))^{2}}{2 T_{\sigma}(\boldsymbol{r})}\right),
\end{gathered}
$$

with the restriction $T_{\sigma}=T_{\sigma^{\prime}}$ for every pair $\sigma, \sigma^{\prime}$. This implies that, given $\sigma$ and $\sigma^{\prime}$,

$$
C_{\sigma \sigma^{\prime}}\left[f_{\sigma}, f_{\sigma^{\prime}}\right]=0
$$

if and only if $f_{\sigma}$ and $f_{\sigma^{\prime}}$ are of the form (A.3) with $T_{\sigma}=T_{\sigma^{\prime}}$.

Other well-known property, derived from (A.4), is $C_{\sigma \sigma^{\prime}}\left[\frac{m_{\sigma}}{T_{\sigma}} \boldsymbol{v} f_{M \sigma}, f_{M \sigma^{\prime}}\right]+C_{\sigma \sigma^{\prime}}\left[f_{M \sigma}, \frac{m_{\sigma^{\prime}}}{T_{\sigma^{\prime}}} \boldsymbol{v} f_{M \sigma^{\prime}}\right]=0$.

Let us take $f_{\sigma}=f_{M \sigma}+\delta f_{\sigma 1}+O\left(\delta^{2}\right)$ for a small parameter $\delta$ and write the lowest-order contribution to (A.2),

$$
\begin{aligned}
s= & -\delta^{2} \sum_{\sigma, \sigma^{\prime}} \int \frac{f_{\sigma 1}}{f_{M \sigma}}\left(C_{\sigma \sigma^{\prime}}\left[f_{M \sigma}, f_{\sigma^{\prime} 1}\right]+C_{\sigma \sigma^{\prime}}\left[f_{\sigma 1}, f_{M \sigma^{\prime}}\right]\right) \mathrm{d}^{3} v \\
& +O\left(\delta^{3}\right) .
\end{aligned}
$$

Since $s=0$ if and only if all the distribution functions are Maxwellians with the same temperature and flow, then

$$
\sum_{\sigma, \sigma^{\prime}} \int \frac{f_{\sigma 1}}{f_{M \sigma}}\left(C_{\sigma \sigma^{\prime}}\left[f_{M \sigma}, f_{\sigma^{\prime} 1}\right]+C_{\sigma \sigma^{\prime}}\left[f_{\sigma 1}, f_{M \sigma^{\prime}}\right]\right) \mathrm{d}^{3} v=0
$$

if and only if, for some $a_{\sigma, 0}(r), a_{1}(r), a_{2}(r)$, the distribution functions $f_{\sigma 1}$ are of the form

$$
f_{\sigma 1}=\left(a_{\sigma, 0}(\boldsymbol{r})+m_{\sigma} \boldsymbol{a}_{1}(\boldsymbol{r}) \cdot \boldsymbol{v}+m_{\sigma} a_{2}(\boldsymbol{r}) \boldsymbol{v}^{2}\right) f_{M \sigma}(\boldsymbol{r}, \boldsymbol{v}),
$$

where the temperature and flow of $f_{M \sigma}$ are the same for all species.

It is useful to have the explicit translation of some of the properties of the collision operator into our non-dimensional variables. With the definition (27) we have

$$
\begin{gathered}
\int \underline{C_{\sigma \sigma^{\prime}}} \mathrm{d}^{3} \underline{v}=0 \\
\int \underline{v} \underline{C_{\sigma \sigma^{\prime}}} \mathrm{d}^{3} \underline{v}=-\int \underline{v} \underline{C_{\sigma^{\prime} \sigma}} \mathrm{d}^{3} \underline{v} \\
\int \frac{1}{2} \tau_{\sigma} \underline{v}^{2} \underline{C_{\sigma \sigma^{\prime}}} \mathrm{d}^{3} \underline{v}=-\int \frac{1}{2} \tau_{\sigma^{\prime}} \underline{v}^{2} \underline{C_{\sigma^{\prime} \sigma}} \mathrm{d}^{3} \underline{v} .
\end{gathered}
$$

Also,

$$
\underline{C_{\sigma \sigma^{\prime}}}\left[\underline{f_{M \sigma}}, \underline{f_{M \sigma^{\prime}}}\right]=0
$$

when

$\underline{f_{M \sigma}}(\underline{\boldsymbol{r}}, \underline{\boldsymbol{v}})=\frac{\underline{n_{\sigma}}(\underline{\boldsymbol{r}})}{\left(2 \pi \underline{T_{\sigma}}(\underline{\boldsymbol{r}})\right)^{3 / 2}} \exp \left(-\frac{\left(\underline{\boldsymbol{v}}-\underline{\boldsymbol{V}}(\underline{\boldsymbol{r}}) / \tau_{\sigma}\right)^{2}}{2 \underline{T_{\sigma}}(\underline{\boldsymbol{r}})}\right)$,

$\underline{f_{M \sigma^{\prime}}}(\underline{\boldsymbol{r}}, \underline{\boldsymbol{v}})=\frac{n_{\sigma^{\prime}}(\underline{\boldsymbol{r}})}{\left(2 \pi \underline{T_{\sigma^{\prime}}}(\underline{\boldsymbol{r}})\right)^{3 / 2}} \exp \left(-\frac{\left(\underline{\boldsymbol{v}}-\underline{\boldsymbol{V}}(\underline{\boldsymbol{r}}) / \tau_{\sigma^{\prime}}\right)^{2}}{2 \underline{T_{\sigma^{\prime}}}(\underline{\boldsymbol{r}})}\right)$,

and $\underline{T_{\sigma}}(\underline{\boldsymbol{r}})=\underline{T_{\sigma^{\prime}}}(\underline{\boldsymbol{r}})$ at every point. Finally,

$\underline{C_{\sigma \sigma^{\prime}}}\left[\frac{1}{\tau_{\sigma} \underline{T_{\sigma}}} \underline{\boldsymbol{v}} \underline{f_{M \sigma}}, \underline{f_{M \sigma^{\prime}}}\right]+\underline{C_{\sigma \sigma^{\prime}}}\left[\underline{f_{M \sigma}}, \frac{1}{\tau_{\sigma^{\prime}} \underline{T_{\sigma^{\prime}}}} \underline{\underline{v}} \underline{f_{M \sigma^{\prime}}}\right]=0$.
Appendix B. Explicit expressions for some pieces of the collision term

Reference [14] defines

$$
\begin{aligned}
C_{\sigma \sigma^{\prime}}^{(1) \mathrm{lw}} & =C_{\sigma \sigma^{\prime}}\left[\frac{1}{T_{\sigma}} \boldsymbol{v} \cdot\left(\boldsymbol{V}_{\sigma}^{p}+\left(\frac{v^{2}}{2 T_{\sigma}}-\frac{5}{2}\right) \boldsymbol{V}_{\sigma}^{\mathrm{T}}\right) \mathcal{T}_{\sigma, 0}^{-1 *} F_{\sigma 0}\right. \\
& \left.+\mathcal{T}_{\sigma, 0}^{-1 *} G_{\sigma 1}, \mathcal{T}_{\sigma^{\prime}, 0}^{-1 *} F_{\sigma^{\prime} 0}\right] \\
& +\frac{\lambda_{\sigma}}{\lambda_{\sigma^{\prime}}} C_{\sigma \sigma^{\prime}}\left[\mathcal{T}_{\sigma, 0}^{-1 *} F_{\sigma 0}, \frac{1}{T_{\sigma^{\prime}}} \boldsymbol{v} \cdot\left(\boldsymbol{V}_{\sigma^{\prime}}^{p}\right.\right. \\
& \left.\left.+\left(\frac{v^{2}}{2 T_{\sigma^{\prime}}}-\frac{5}{2}\right) \boldsymbol{V}_{\sigma^{\prime}}^{\mathrm{T}}\right) \mathcal{T}_{\sigma^{\prime}, 0}^{-1 *} F_{\sigma^{\prime} 0}+\mathcal{T}_{\sigma^{\prime}, 0}^{-1 *} G_{\sigma^{\prime} 1}\right]
\end{aligned}
$$

and

$$
\begin{aligned}
C_{\sigma \sigma^{\prime}}^{(1) \mathrm{sw}} & =C_{\sigma \sigma^{\prime}}\left[\mathbb{T}_{\sigma, 0} F_{\sigma 1}^{\mathrm{sw}}\right. \\
- & \left.\frac{Z_{\sigma} \lambda_{\sigma}}{T_{\sigma}} \mathbb{T}_{\sigma, 0} \tilde{\phi}_{\sigma 1}^{\mathrm{sw}} \mathcal{T}_{\sigma, 0}^{-1 *} F_{\sigma 0}, \mathcal{T}_{\sigma^{\prime}, 0}^{-1 *} F_{\sigma^{\prime} 0}\right] \\
+ & \frac{\lambda_{\sigma}}{\lambda_{\sigma^{\prime}}} C_{\sigma \sigma^{\prime}}\left[\mathcal{T}_{\sigma, 0}^{-1 *} F_{\sigma 0}, \mathbb{T}_{\sigma^{\prime}, 0} F_{\sigma^{\prime} 1}^{\mathrm{sw}}\right. \\
- & \left.\frac{Z_{\sigma^{\prime}} \lambda_{\sigma^{\prime}}}{T_{\sigma^{\prime}}} \mathbb{T}_{\sigma^{\prime}, 0} \tilde{\phi}_{\sigma^{\prime} 1}^{\mathrm{sw}} \mathcal{T}_{\sigma^{\prime}, 0}^{-1 *} F_{\sigma^{\prime} 0}\right]
\end{aligned}
$$

Therefore,

$$
\begin{aligned}
& {\left[\mathcal{T}_{\sigma, 1}^{*} C_{\sigma \sigma^{\prime}}^{(1)}\right]^{\mathrm{lw}}=\left(\rho \cdot \nabla_{\boldsymbol{R}}+\hat{u}_{1} \partial_{u}+\hat{\mu}_{1}^{\mathrm{lw}} \partial_{\mu}+\hat{\theta}_{1}^{\mathrm{lw}} \partial_{\theta}\right) \mathcal{T}_{\sigma, 0}^{*} C_{\sigma \sigma^{\prime}}^{(1) l \mathrm{w}}} \\
& \quad+\left[\mathcal{T}_{\sigma, 1}^{*} C_{\sigma \sigma^{\prime}}^{(1) \mathrm{sw}}\right]^{\mathrm{lw}} .
\end{aligned}
$$

The first-order coordinate transformation that enters expression (B.3) is given in appendix C; in particular, $\hat{u}_{1}, \hat{\mu}_{1}^{\mathrm{lw}}, \hat{\theta}_{1}^{\mathrm{lw}}$ are defined in (C.2) and (C.3). We use those results and

$$
\begin{gathered}
B^{-1} \nabla_{\boldsymbol{R}} \cdot(B \boldsymbol{\rho})+\partial_{u} \hat{u}_{1}+\partial_{\mu} \hat{\mu}_{1}+\partial_{\theta} \hat{\theta}_{1} \\
\quad=\frac{u}{B} \hat{\boldsymbol{b}} \cdot \nabla_{\boldsymbol{R}} \times \hat{\boldsymbol{b}}
\end{gathered}
$$

to write

$$
\begin{gathered}
\left\langle\left[\mathcal{T}_{\sigma, 1}^{*} C_{\sigma \sigma^{\prime}}^{(1)}\right]^{\mathrm{lw}}\right\rangle=\partial_{u}\left(\left\langle u \hat{\boldsymbol{b}} \cdot \nabla_{\boldsymbol{R}} \hat{\boldsymbol{b}} \cdot \boldsymbol{\rho} \mathcal{T}_{\sigma, 0}^{*} C_{\sigma \sigma^{\prime}}^{(1) \mathrm{lw}}\right\rangle\right. \\
\left.-\mu \hat{\boldsymbol{b}} \cdot \nabla_{\boldsymbol{R}} \times \hat{\boldsymbol{b}}\left\langle\mathcal{T}_{\sigma, 0}^{*} C_{\sigma \sigma^{\prime}}^{(1) \mathrm{w}}\right\rangle\right) \\
+\partial_{\mu}\left\{\frac{u \mu}{B} \hat{\boldsymbol{b}} \cdot \nabla_{\boldsymbol{R}} \times \hat{\boldsymbol{b}}\left\langle\mathcal{T}_{\sigma, 0}^{*} C_{\sigma \sigma^{\prime}}^{(1) \mathrm{lw}}\right\rangle\right. \\
-\left\langle\left(\frac{Z_{\sigma}}{B} \boldsymbol{\rho} \cdot \nabla_{\boldsymbol{R}} \varphi_{0}+\frac{\mu}{B} \boldsymbol{\rho} \cdot \nabla_{\boldsymbol{R}} B\right.\right. \\
\left.\left.\left.+\frac{u^{2}}{B} \hat{\boldsymbol{b}} \cdot \nabla \hat{\boldsymbol{b}} \cdot \boldsymbol{\rho}\right) \mathcal{T}_{\sigma, 0}^{*} C_{\sigma \sigma^{\prime}}^{(1) l \mathrm{w}}\right\rangle\right\}
\end{gathered}
$$




$$
\begin{aligned}
& -\frac{u}{B} \hat{\boldsymbol{b}} \cdot \nabla_{\boldsymbol{R}} \times \hat{\boldsymbol{b}}\left\langle\mathcal{T}_{\sigma, 0}^{*} C_{\sigma \sigma^{\prime}}^{(1) \mathrm{lw}}\right\rangle \\
& +\frac{1}{B} \nabla_{\boldsymbol{R}} \cdot\left\langle B \boldsymbol{\rho} \mathcal{T}_{\sigma, 0}^{*} C_{\sigma \sigma^{\prime}}^{(1) \mathrm{lw}}\right\rangle+\left\langle\left[\mathcal{T}_{\sigma, 1}^{*} C_{\sigma \sigma^{\prime}}^{(1) \mathrm{sw}}\right]^{\mathrm{lw}}\right\rangle .
\end{aligned}
$$

The turbulent piece $\left[\mathcal{T}_{\sigma, 1}^{*} C_{\sigma \sigma^{\prime}}^{\mathrm{sw}}\right]^{\text {lw }}$ appearing in (B.3) was computed in appendix $\mathrm{E}$ of [14]. We collect here the result,

$\left[\mathcal{T}_{\sigma, 1}^{*} C_{\sigma \sigma^{\prime}}^{\mathrm{sw}}\right]^{\mathrm{lw}}=\left[\frac{Z_{\sigma} \lambda_{\sigma}}{B}\left(-\tilde{\phi}_{\sigma 1}^{\mathrm{sw}} \partial_{\mu}+\partial_{\mu} \tilde{\Phi}_{\sigma 1}^{\mathrm{sw}} \partial_{\theta}\right)\right.$

$\times\left\{\mathcal{T}_{\mathrm{NP}, \sigma}^{*} C_{\sigma \sigma^{\prime}}\left[\mathbb{T}_{\sigma, 0} F_{\sigma 1}^{\mathrm{sw}}-\frac{Z_{\sigma} \lambda_{\sigma}}{T_{\sigma}} \mathbb{T}_{\sigma, 0} \tilde{\phi}_{\sigma 1}^{\mathrm{sw}} \mathcal{T}_{\sigma, 0}^{-1 *} F_{\sigma 0}, \mathcal{T}_{\sigma^{\prime}, 0}^{-1 *} F_{\sigma^{\prime} 0}\right]\right.$

$+\frac{\lambda_{\sigma}}{\lambda_{\sigma^{\prime}}} \mathcal{T}_{\mathrm{NP}, \sigma}^{*} C_{\sigma \sigma^{\prime}}\left[\mathcal{T}_{\sigma, 0}^{-1 *} F_{\sigma 0}, \mathbb{T}_{\sigma^{\prime}, 0} F_{\sigma^{\prime} 1}^{\mathrm{sw}}\right.$

$\left.\left.\left.-\frac{Z_{\sigma^{\prime}} \lambda_{\sigma^{\prime}}}{T_{\sigma^{\prime}}} \mathbb{T}_{\sigma^{\prime}, 0} \tilde{\phi}_{\sigma^{\prime} 1}^{\mathrm{sw}} \mathcal{T}_{\sigma^{\prime}, 0}^{-1 *} F_{\sigma^{\prime} 0}\right]\right\}\right]^{\mathrm{lw}}$.

As for its gyroaverage,

$\left\langle\left[\mathcal{T}_{\sigma, 1}^{*} C_{\sigma \sigma^{\prime}}^{\mathrm{sw}}\right]^{\mathrm{lw}}\right\rangle=-\partial_{\mu}\left\langle\left[\frac{Z_{\sigma} \lambda_{\sigma}}{B} \tilde{\phi}_{\sigma 1}^{\mathrm{sw}}\right.\right.$

$\times\left\{\mathcal{T}_{\mathrm{NP}, \sigma}^{*} C_{\sigma \sigma^{\prime}}\left[\mathbb{T}_{\sigma, 0} F_{\sigma 1}^{\mathrm{sw}}-\frac{Z_{\sigma} \lambda_{\sigma}}{T_{\sigma}} \mathbb{T}_{\sigma, 0} \tilde{\phi}_{\sigma 1}^{\mathrm{sw}} \mathcal{T}_{\sigma, 0}^{-1 *} F_{\sigma 0}, \mathcal{T}_{\sigma^{\prime}, 0}^{-1 *} F_{\sigma^{\prime} 0}\right]\right.$

$+\frac{\lambda_{\sigma}}{\lambda_{\sigma^{\prime}}} \mathcal{T}_{\mathrm{NP}, \sigma}^{*} C_{\sigma \sigma^{\prime}}\left[\mathcal{T}_{\sigma, 0}^{-1 *} F_{\sigma 0}, \mathbb{T}_{\sigma^{\prime}, 0} F_{\sigma^{\prime} 1}^{\mathrm{sw}}\right.$

$\left.\left.\left.\left.-\frac{Z_{\sigma^{\prime}} \lambda_{\sigma^{\prime}}}{T_{\sigma^{\prime}}} \mathbb{T}_{\sigma^{\prime}, 0} \tilde{\phi}_{\sigma^{\prime} 1}^{\mathrm{sw}} \mathcal{T}_{\sigma^{\prime}, 0}^{-1 *} F_{\sigma^{\prime} 0}\right]\right\}\right]^{\mathrm{lw}}\right\rangle$.

In order to get the last expression we have integrated by parts in $\theta$ and $\mu$.

The full computation of the last term in equation (79) is tedious and was performed in appendix $F$ of [14]. The necessary result is

$$
\begin{aligned}
& \left\langle\mathcal{T}_{\sigma, 0}^{*} C_{\sigma \sigma^{\prime}}^{(2) \mathrm{w}}\right\rangle=\mathcal{T}_{\sigma, 0}^{*} C_{\sigma \sigma^{\prime}}\left[\mathcal{T}_{\sigma, 0}^{-1 *}\left\langle F_{\sigma 2}^{\mathrm{lw}}\right\rangle+\mathcal{T}_{\sigma, 0}^{-1 *}\left\langle\mathcal{T}_{\sigma, 0}^{*}\left[\mathcal{T}_{\sigma, 1}^{-1 *} F_{\sigma 1}^{\mathrm{lw}}\right]^{\mathrm{lw}}\right\rangle\right. \\
& \left.+\mathcal{T}_{\sigma, 0}^{-1 *}\left\langle\mathcal{T}_{\sigma, 0}^{*}\left[\mathcal{T}_{\sigma, 2}^{-1 *} F_{\sigma 0}\right]^{\mathrm{lw}}\right\rangle, \mathcal{T}_{\sigma^{\prime}, 0}^{-1 *} F_{\sigma^{\prime} 0}\right] \\
& +\left(\frac{\lambda_{\sigma}}{\lambda_{\sigma^{\prime}}}\right)^{2} \mathcal{T}_{\sigma, 0}^{*} C_{\sigma \sigma^{\prime}}\left[\mathcal{T}_{\sigma, 0}^{-1 *} F_{\sigma 0},\right. \\
& \mathcal{T}_{\sigma^{\prime}, 0}^{-1 *}\left\langle F_{\sigma^{\prime} 2}^{\mathrm{lw}}\right\rangle+\mathcal{T}_{\sigma^{\prime}, 0}^{-1 *}\left\langle\mathcal{T}_{\sigma^{\prime}, 0}^{*}\left[\mathcal{T}_{\sigma^{\prime}, 1}^{-1 *} F_{\sigma^{\prime} 1}^{\mathrm{lw}}\right]^{\mathrm{lw}}\right\rangle \\
& \left.+\mathcal{T}_{\sigma^{\prime}, 0}^{-1 *}\left\langle\mathcal{T}_{\sigma^{\prime}, 0}^{*}\left[\mathcal{T}_{\sigma^{\prime}, 2}^{-1 *} F_{\sigma^{\prime} 0}\right]^{\mathrm{lw}}\right\rangle\right] \\
& +\frac{\lambda_{\sigma}}{\lambda_{\sigma^{\prime}}}\left\langle\mathcal { T } _ { \sigma , 0 } ^ { * } C _ { \sigma \sigma ^ { \prime } } \left[\mathcal{T}_{\sigma, 0}^{-1 *} F_{\sigma 1}^{\mathrm{lw}}+\left[\mathcal{T}_{\sigma, 1}^{-1 *} F_{\sigma 0}\right]^{\mathrm{lw}},\right.\right. \\
& \left.\left.\mathcal{T}_{\sigma^{\prime}, 0}^{-1 *} F_{\sigma^{\prime} 1}^{\mathrm{lw}}+\left[\mathcal{T}_{\sigma^{\prime}, 1}^{-1 *} F_{\sigma^{\prime} 0}\right]^{\mathrm{lw}}\right]\right\rangle+\frac{\lambda_{\sigma}}{\lambda_{\sigma^{\prime}}}\left\langle\mathcal { T } _ { \sigma , 0 } ^ { * } \left[C _ { \sigma \sigma ^ { \prime } } \left[\mathbb{T}_{\sigma, 0} F_{\sigma 1}^{\mathrm{sw}}\right.\right.\right. \\
& -\frac{Z_{\sigma} \lambda_{\sigma}}{T_{\sigma}} \mathbb{T}_{\sigma, 0} \widetilde{\phi}_{\sigma 1}^{\mathrm{sw}} \mathcal{T}_{\sigma, 0}^{-1 *} F_{\sigma 0}, \mathbb{T}_{\sigma^{\prime}, 0} F_{\sigma^{\prime} 1}^{\mathrm{sw}} \\
& \left.\left.\left.-\frac{Z_{\sigma^{\prime}} \lambda_{\sigma^{\prime}}}{T_{\sigma^{\prime}}} \mathbb{T}_{\sigma^{\prime}, 0} \widetilde{\phi}_{\sigma^{\prime} 1}^{\mathrm{sw}} \mathcal{T}_{\sigma^{\prime}, 0}^{-1 *} F_{\sigma^{\prime} 0}\right]\right]^{\mathrm{lw}}\right\rangle .
\end{aligned}
$$

Here,

$\left\langle\mathcal{T}_{\sigma, 0}^{*}\left[\mathcal{T}_{\sigma, 1}^{-1 *} F_{\sigma 1}^{1 \mathrm{w}}\right]^{1 \mathrm{w}}\right\rangle=\mu \hat{\boldsymbol{b}} \cdot \nabla_{\boldsymbol{R}} \times \hat{\boldsymbol{b}}\left(\partial_{u}-\frac{u}{B} \partial_{\mu}\right) F_{\sigma 1}^{\mathrm{lw}}$,

$\left\langle\mathcal{T}_{\sigma, 0}^{*}\left[\mathcal{T}_{\sigma, 2}^{-1 *} F_{\sigma 0}\right]^{\mathrm{lw}}\right\rangle=\frac{\mu}{2 B}(\stackrel{\leftrightarrow}{\boldsymbol{I}}-\hat{\boldsymbol{b}} \hat{\boldsymbol{b}}):\left[\nabla_{\boldsymbol{R}} \nabla_{\boldsymbol{R}} \ln n_{\sigma}\right.$

$\left.+\left(\frac{u^{2} / 2+\mu B}{T_{\sigma}}-\frac{3}{2}\right) \nabla_{R} \nabla_{R} \ln T_{\sigma}\right] F_{\sigma 0}$

$-\frac{\mu}{B} \frac{Z_{\sigma}}{T_{\sigma}^{2}} \nabla_{R} \varphi_{0} \cdot \nabla_{R} T_{\sigma} F_{\sigma 0}-\frac{\mu}{2 B} \frac{u^{2} / 2+\mu B}{T_{\sigma}^{3}}\left|\nabla_{R} T_{\sigma}\right|^{2} F_{\sigma 0}$

$+\frac{\mu}{2 B} \mid \frac{\nabla_{R^{n}} n_{\sigma}}{n_{\sigma}}+\frac{Z_{\sigma} \nabla_{R} \varphi_{0}}{T_{\sigma}}$

$+\left.\left(\frac{u^{2} / 2+\mu B}{T_{\sigma}}-\frac{3}{2}\right) \frac{\nabla_{R} T_{\sigma}}{T_{\sigma}}\right|^{2} F_{\sigma 0}$

$-\frac{\mu}{2 B^{2}} \nabla_{\boldsymbol{R}_{\perp}} B \cdot\left(\frac{\nabla_{\boldsymbol{R}} n_{\sigma}}{n_{\sigma}}+\frac{Z_{\sigma} \nabla_{\boldsymbol{R}} \varphi_{0}}{T_{\sigma}}\right.$

$\left.+\left(\frac{u^{2} / 2+\mu B}{T_{\sigma}}-\frac{3}{2}\right) \frac{\nabla_{R} T_{\sigma}}{T_{\sigma}}\right) F_{\sigma 0}$

$+\frac{Z_{\sigma}^{2} \lambda_{\sigma}^{2}}{2 T_{\sigma}^{2}}\left[\left\langle\left(\widetilde{\phi}_{\sigma 1}^{\mathrm{sw}}\right)^{2}\right\rangle\right]^{1 \mathrm{w}} F_{\sigma 0}+\frac{1}{T_{\sigma}}\left[-\frac{Z_{\sigma}^{2}}{2 B^{2}}\left|\nabla_{R} \varphi_{0}\right|^{2}\right.$

$-\frac{Z_{\sigma}^{2} \lambda_{\sigma}^{2}}{2 B} \partial_{\mu}\left[\left\langle\left(\widetilde{\phi}_{\sigma 1}^{\mathrm{sw}}\right)^{2}\right\rangle\right]^{1 \mathrm{w}}-\frac{3 Z_{\sigma} \mu}{2 B^{2}} \nabla_{\boldsymbol{R}_{\perp}} B \cdot \nabla_{\boldsymbol{R}} \varphi_{0}$

$-\frac{Z_{\sigma} u^{2}}{B^{2}} \hat{\boldsymbol{b}} \cdot \nabla_{\boldsymbol{R}} \hat{\boldsymbol{b}} \cdot \nabla_{R} \varphi_{0}+\Psi_{B, \sigma}$

$\left.+\frac{Z_{\sigma} \mu}{B}(\stackrel{\leftrightarrow}{\boldsymbol{I}}-\hat{\boldsymbol{b}} \hat{\boldsymbol{b}}): \nabla_{\boldsymbol{R}} \nabla_{\boldsymbol{R}} \varphi_{0}\right] F_{\sigma 0}$,

and $\mathcal{T}_{\sigma, 1}^{-1 *}$ is obtained from the results of appendix $\mathrm{C}$.

Finally, the last term in equation (99) is given by

$\left\langle\mathcal{T}_{\sigma, 0}^{*} \mathbb{C}_{\sigma \sigma^{\prime}}\right\rangle=\left\langle\mathcal{T}_{\sigma, 0}^{*} C_{\sigma \sigma^{\prime}}^{(2) \mathrm{lw}}\right\rangle-\mathcal{T}_{\sigma, 0}^{*} C_{\sigma \sigma^{\prime}}\left[\mathcal{T}_{\sigma, 0}^{-1 *} G_{\sigma 2}^{\mathrm{lw}}, \mathcal{T}_{\sigma^{\prime}, 0}^{-1 *} F_{\sigma^{\prime} 0}\right]$

$-\left(\frac{\lambda_{\sigma}}{\lambda_{\sigma}^{\prime}}\right)^{2} C_{\sigma \sigma^{\prime}}\left[\mathcal{T}_{\sigma, 0}^{-1 *} F_{\sigma 0}, \mathcal{T}_{\sigma^{\prime}, 0}^{-1 *} G_{\sigma^{\prime} 2}^{\mathrm{lw}}\right]$.

Explicitly,

$$
\begin{aligned}
& \left\langle\mathcal{T}_{\sigma, 0}^{*} \mathbb{C}_{\sigma \sigma^{\prime}}\right\rangle=\mathcal{T}_{\sigma, 0}^{*} C_{\sigma \sigma^{\prime}}\left[\mathcal{T}_{\sigma, 0}^{-1 *}\left\langle\mathcal{T}_{\sigma, 0}^{*}\left[\mathcal{T}_{\sigma, 1}^{-1 *} F_{\sigma 1}^{\mathrm{lw}}\right]^{1 \mathrm{w}}\right\rangle\right. \\
& +\mathcal{T}_{\sigma, 0}^{-1 *}\left\langle\mathcal{T}_{\sigma, 0}^{*}\left[\mathcal{T}_{\sigma, 2}^{-1 *} F_{\sigma 0}\right]^{\mathrm{lw}}\right\rangle \\
& +\mathcal{T}_{\sigma, 0}^{-1 *}\left\{-\frac{1}{T_{\sigma}}\left(\frac{Z_{\sigma} \mu}{2 B}(\stackrel{\leftrightarrow}{\boldsymbol{I}}-\hat{\boldsymbol{b}} \hat{\boldsymbol{b}}): \nabla_{\boldsymbol{R}} \nabla_{\boldsymbol{R}} \varphi_{0}\right.\right. \\
& \left.+\Psi_{B, \sigma}+Z_{\sigma} \lambda_{\sigma} \Psi_{\phi B, \sigma}^{\mathrm{lw}}+Z_{\sigma}^{2} \lambda_{\sigma}^{2} \Psi_{\phi, \sigma}^{\mathrm{lw}}\right) F_{\sigma 0} \\
& \left.\left.+\frac{Z_{\sigma} \lambda_{\sigma} \varphi_{1}^{\mathrm{lw}}}{u} \partial_{u} G_{\sigma 1}^{1 \mathrm{w}}\right\}, \mathcal{T}_{\sigma^{\prime}, 0}^{-1 *} F_{\sigma^{\prime} 0}\right] \\
& +\left(\frac{\lambda_{\sigma}}{\lambda_{\sigma^{\prime}}}\right)^{2} \mathcal{T}_{\sigma, 0}^{*} C_{\sigma \sigma^{\prime}}\left[\mathcal{T}_{\sigma, 0}^{-1 *} F_{\sigma 0},\right.
\end{aligned}
$$




$$
\begin{aligned}
& \mathcal{T}_{\sigma^{\prime}, 0}^{-1 *}\left\langle\mathcal{T}_{\sigma^{\prime}, 0}^{*}\left[\mathcal{T}_{\sigma^{\prime}, 1}^{-1 *} F_{\sigma^{\prime} 1}^{\mathrm{lw}}\right]^{\mathrm{lw}}\right\rangle+\mathcal{T}_{\sigma^{\prime}, 0}^{-1 *}\left\langle\mathcal{T}_{\sigma^{\prime}, 0}^{*}\left[\mathcal{T}_{\sigma^{\prime}, 2}^{-1 *} F_{\sigma^{\prime} 0}\right]^{\mathrm{lw}}\right\rangle \\
&+ \mathcal{T}_{\sigma^{\prime}, 0}^{-1 *}\left\{-\frac{1}{T_{\sigma^{\prime}}}\left(\frac{Z_{\sigma^{\prime}} \mu}{2 B}(\stackrel{\leftrightarrow}{\boldsymbol{I}}-\hat{\boldsymbol{b}} \hat{\boldsymbol{b}}): \nabla_{R} \nabla_{R} \varphi_{0}\right.\right. \\
&+\left.\Psi_{B, \sigma^{\prime}}+Z_{\sigma^{\prime}} \lambda_{\sigma^{\prime}} \Psi_{\phi B, \sigma^{\prime}}^{\mathrm{lw}}+Z_{\sigma^{\prime}}^{2} \lambda_{\sigma^{\prime}}^{2} \Psi_{\phi, \sigma^{\prime}}^{\mathrm{lw}}\right) F_{\sigma^{\prime} 0} \\
&+\frac{\left.\left.Z_{\sigma}^{\prime} \lambda_{\sigma}^{\prime} \varphi_{1}^{\mathrm{lw}} \partial_{u} G_{\sigma^{\prime} 1}^{1 \mathrm{w}}\right\}\right]}{u} \\
&+\frac{\lambda_{\sigma}}{\lambda_{\sigma^{\prime}}}\left\langle\mathcal { T } _ { \sigma , 0 } ^ { * } C _ { \sigma \sigma ^ { \prime } } \left[\mathcal{T}_{\sigma, 0}^{-1 *} F_{\sigma 1}^{\mathrm{lw}}+\left[\mathcal{T}_{\sigma, 1}^{-1 *} F_{\sigma 0}\right]^{\mathrm{lw}},\right.\right. \\
& \mathcal{T}_{\sigma^{\prime}, 0}^{-1 *}\left.\left.F_{\sigma^{\prime} 1}^{\mathrm{lw}}+\left[\mathcal{T}_{\sigma^{\prime}, 1}^{-1 *} F_{\sigma^{\prime} 0}\right]^{\mathrm{lw}}\right]\right\rangle \\
&+ \frac{\lambda_{\sigma}}{\lambda_{\sigma^{\prime}}}\left\langle\mathcal { T } _ { \sigma , 0 } ^ { * } \left[C _ { \sigma \sigma ^ { \prime } } \left[\mathbb{T}_{\sigma, 0} F_{\sigma 1}^{\mathrm{sw}}-\frac{Z_{\sigma} \lambda_{\sigma}}{T_{\sigma}} \mathbb{T}_{\sigma, 0} \widetilde{\phi}_{\sigma 1}^{\mathrm{sw}} \mathcal{T}_{\sigma, 0}^{-1 *} F_{\sigma 0},\right.\right.\right. \\
&\left.\left.\left.\mathbb{T}_{\sigma^{\prime}, 0} F_{\sigma^{\prime} 1}^{\mathrm{sw}}-\frac{Z_{\sigma^{\prime}} \lambda_{\sigma^{\prime}}}{T_{\sigma^{\prime}}} \mathbb{T}_{\sigma^{\prime}, 0} \widetilde{\phi}_{\sigma^{\prime} 1}^{\mathrm{sw}} \mathcal{T}_{\sigma^{\prime}, 0}^{-1 *} F_{\sigma^{\prime} 0}\right]\right]^{\mathrm{lw}}\right\rangle .
\end{aligned}
$$

\section{Appendix C. Gyrokinetic transformation to first order}

The explicit expressions for the gyrokinetic transformation $(\boldsymbol{r}, \boldsymbol{v})=\mathcal{T}_{\sigma}(\boldsymbol{R}, u, \mu, \theta, t)$ to order $\epsilon_{\sigma}$ were derived in detail in appendix $C$ of [14]. Here, we only quote the results. Namely,

$$
\begin{gathered}
r=\boldsymbol{R}+\epsilon_{\sigma} \boldsymbol{\rho}+O\left(\epsilon_{\sigma}^{2}\right), \\
v_{\|}=u+\epsilon_{\sigma} \hat{u}_{1}+O\left(\epsilon_{\sigma}^{2}\right), \\
\mu_{0}=\mu+\epsilon_{\sigma} \hat{\mu}_{1}+O\left(\epsilon_{\sigma}^{2}\right), \\
\theta_{0}=\theta+\epsilon_{\sigma} \hat{\theta}_{1}+O\left(\epsilon_{\sigma}^{2}\right),
\end{gathered}
$$

where

$$
\begin{aligned}
\hat{u}_{1} & =u \hat{\boldsymbol{b}} \cdot \nabla_{R} \hat{\boldsymbol{b}} \cdot \boldsymbol{\rho}+\frac{B}{4}[\boldsymbol{\rho}(\boldsymbol{\rho} \times \hat{\boldsymbol{b}})+(\boldsymbol{\rho} \times \hat{\boldsymbol{b}}) \boldsymbol{\rho}]: \nabla_{\boldsymbol{R}} \hat{\boldsymbol{b}} \\
& -\mu \hat{\boldsymbol{b}} \cdot \nabla_{\boldsymbol{R}} \times \hat{\boldsymbol{b}} \\
\hat{\mu}_{1} & =-\frac{\mu}{B} \boldsymbol{\rho} \cdot \nabla_{R} B-\frac{u}{4}(\boldsymbol{\rho}(\boldsymbol{\rho} \times \hat{\boldsymbol{b}})+(\boldsymbol{\rho} \times \hat{\boldsymbol{b}}) \boldsymbol{\rho}): \nabla_{R} \hat{\boldsymbol{b}} \\
& +\frac{u \mu}{B} \hat{\boldsymbol{b}} \cdot \nabla_{R} \times \hat{\boldsymbol{b}}-\frac{u^{2}}{B} \hat{\boldsymbol{b}} \cdot \nabla_{R} \hat{\boldsymbol{b}} \cdot \boldsymbol{\rho}-\frac{Z_{\sigma} \lambda_{\sigma}}{B} \tilde{\phi}_{\sigma 1}, \\
\hat{\theta}_{1} & =(\boldsymbol{\rho} \times \hat{\boldsymbol{b}}) \cdot\left(\nabla_{R} \ln B+\frac{u^{2}}{2 \mu B} \hat{\boldsymbol{b}} \cdot \nabla_{R} \hat{\boldsymbol{b}}\right. \\
& \left.-\hat{\boldsymbol{b}} \times \nabla_{\boldsymbol{R}} \hat{e}_{2} \cdot \hat{\boldsymbol{e}}_{1}\right)-\frac{u}{8 \mu}(\boldsymbol{\rho} \boldsymbol{\rho}-(\boldsymbol{\rho} \times \hat{\boldsymbol{b}})(\boldsymbol{\rho} \times \hat{\boldsymbol{b}})): \nabla_{\boldsymbol{R}} \hat{\boldsymbol{b}} \\
& +\frac{u}{2 B^{2}} \hat{\boldsymbol{b}} \cdot \nabla_{R} B+\frac{Z_{\sigma} \lambda_{\sigma}}{B} \partial_{\mu} \tilde{\Phi}_{\sigma 1} \cdot
\end{aligned}
$$

In the main text of this paper we need the long-wavelength limit of the previous expressions. Using (52) and (53) we get

$$
\begin{aligned}
\hat{u}_{1}^{\mathrm{lw}} & =\hat{u}_{1} \\
\hat{\mu}_{1}^{\mathrm{lw}} & =-\frac{\mu}{B} \boldsymbol{\rho} \cdot \nabla_{\boldsymbol{R}} B-\frac{u}{4}(\boldsymbol{\rho}(\boldsymbol{\rho} \times \hat{\boldsymbol{b}})+(\boldsymbol{\rho} \times \hat{\boldsymbol{b}}) \boldsymbol{\rho}): \nabla_{\boldsymbol{R}} \hat{\boldsymbol{b}} \\
& +\frac{u \mu}{B} \hat{\boldsymbol{b}} \cdot \nabla_{\boldsymbol{R}} \times \hat{\boldsymbol{b}}-\frac{u^{2}}{B} \hat{\boldsymbol{b}} \cdot \nabla_{\boldsymbol{R}} \hat{\boldsymbol{b}} \cdot \boldsymbol{\rho}-\frac{Z_{\sigma}}{B} \boldsymbol{\rho} \cdot \nabla_{\boldsymbol{R}} \varphi_{0}, \\
\hat{\theta}_{1}^{\mathrm{lw}} & =(\boldsymbol{\rho} \times \hat{\boldsymbol{b}}) \cdot\left(\nabla_{\boldsymbol{R}} \ln B+\frac{u^{2}}{2 \mu B} \hat{\boldsymbol{b}} \cdot \nabla_{\boldsymbol{R}} \hat{\boldsymbol{b}}\right. \\
& \left.-\hat{\boldsymbol{b}} \times \nabla_{\boldsymbol{R}} \hat{\boldsymbol{e}}_{2} \cdot \hat{\boldsymbol{e}}_{1}\right)-\frac{u}{8 \mu}(\boldsymbol{\rho} \boldsymbol{\rho}-(\boldsymbol{\rho} \times \hat{\boldsymbol{b}})(\boldsymbol{\rho} \times \hat{\boldsymbol{b}})): \nabla_{\boldsymbol{R}} \hat{\boldsymbol{b}} \\
& +\frac{u}{2 B^{2}} \hat{\boldsymbol{b}} \cdot \nabla_{\boldsymbol{R}} B+\frac{Z_{\sigma}}{2 \mu B}(\boldsymbol{\rho} \times \hat{\boldsymbol{b}}) \cdot \nabla_{\boldsymbol{R}} \varphi_{0} .
\end{aligned}
$$

Next, we proceed to calculate the long-wavelength limit of $\mathcal{T}_{\sigma}^{-1 *} F_{\sigma 0}$ to first order in $\epsilon_{\sigma}$, employed in section 3.1.3. Inverting (C.1) to first order, and recalling (C.3) and the relations $\partial_{u} F_{\sigma 0}=-\left(u / T_{\sigma}\right) F_{\sigma 0}, \partial_{\mu} F_{\sigma 0}=-\left(B / T_{\sigma}\right) F_{\sigma 0}$, one finds that

$$
\begin{aligned}
& {\left[\mathcal{T}_{\sigma, 1}^{-1 *} F_{\sigma 0}\right]^{1 \mathrm{w}}=\frac{1}{T_{\sigma}}\left[\boldsymbol{v} \cdot \boldsymbol{V}_{\sigma}^{\mathrm{p}}+\left(\frac{v^{2}}{2 T_{\sigma}}-\frac{5}{2}\right) \boldsymbol{v} \cdot \boldsymbol{V}_{\sigma}^{\mathrm{T}}\right.} \\
& \left.+\frac{Z_{\sigma}}{B} \boldsymbol{v} \cdot\left(\hat{\boldsymbol{b}} \times \nabla_{r} \varphi_{0}\right)\right] \mathcal{T}_{\sigma, 0}^{-1 *} F_{\sigma 0},
\end{aligned}
$$

with $\boldsymbol{V}_{\sigma}^{\mathrm{p}}$ and $\boldsymbol{V}_{\sigma}^{\mathrm{T}}$ defined in (65).

\section{Appendix D. A convenient way to write the second-order Fokker-Planck equation}

In this appendix we rewrite equation (79) in a way that is very useful to compute the transport equations for density and energy. Defining $G_{\sigma 2}^{\text {lw }}$ by

$$
\begin{aligned}
\left\langle F_{\sigma 2}^{\mathrm{lw}}\right\rangle & =G_{\sigma 2}^{\mathrm{lw}}-\frac{1}{T_{\sigma}}\left[Z _ { \sigma } \lambda _ { \sigma } ^ { 2 } \left(\varphi_{2}^{\mathrm{lw}}\right.\right. \\
& \left.+\frac{\mu}{2 \lambda_{\sigma}^{2} B}(\stackrel{\leftrightarrow}{\boldsymbol{I}}-\hat{\boldsymbol{b}} \hat{\boldsymbol{b}}): \nabla_{\boldsymbol{R}} \nabla_{R} \varphi_{0}\right) \\
+ & \left.\Psi_{B, \sigma}+Z_{\sigma} \lambda_{\sigma} \Psi_{\phi B, \sigma}^{\mathrm{lw}}+Z_{\sigma}^{2} \lambda_{\sigma}^{2} \Psi_{\phi, \sigma}^{\mathrm{lw}}\right] F_{\sigma 0} \\
& +\frac{1}{2}\left(\frac{Z_{\sigma} \lambda_{\sigma} \varphi_{1}^{\mathrm{lw}}}{T_{\sigma}}\right)^{2} F_{\sigma 0}+\frac{Z_{\sigma} \lambda_{\sigma} \varphi_{1}^{\mathrm{lw}}}{u} \partial_{u} G_{\sigma 1}^{\mathrm{lw}}
\end{aligned}
$$

and using (72), equation (79) becomes

$$
\begin{aligned}
(u \hat{\boldsymbol{b}} & \left.\cdot \nabla_{\boldsymbol{R}}-\mu \hat{\boldsymbol{b}} \cdot \nabla_{\boldsymbol{R}} B \partial_{u}\right) G_{\sigma 2}^{\mathrm{lw}}+\frac{\lambda_{\sigma}^{2}}{\tau_{\sigma}} \partial_{\epsilon_{\mathrm{s}}^{2} t} F_{\sigma 0} \\
& +\left(\boldsymbol{v}_{M}+\boldsymbol{v}_{E, \sigma}^{(0)}\right) \cdot \nabla_{\boldsymbol{R}} G_{\sigma 1}^{\mathrm{lw}} \\
& +u \boldsymbol{\kappa} \cdot\left(\boldsymbol{v}_{\nabla B}+\boldsymbol{v}_{E, \sigma}^{(0)}\right) \partial_{u} G_{\sigma 1}^{\mathrm{lw}} \\
+ & {\left[-\frac{u}{B}\left(\hat{\boldsymbol{b}} \cdot \nabla_{\boldsymbol{R}} \times \hat{\boldsymbol{b}}\right)\left(\boldsymbol{v}_{M}+\boldsymbol{v}_{E, \sigma}^{(0)}\right)\right.}
\end{aligned}
$$




$$
\begin{aligned}
& \left.-\frac{u \mu}{B}\left(\nabla_{R} \times K\right)_{\perp}\right] \cdot \nabla_{R} F_{\sigma 0} \\
& -\left[\frac{u^{2}}{B}\left(\hat{\boldsymbol{b}} \cdot \nabla_{R} \times \hat{\boldsymbol{b}}\right) \kappa\right. \\
& \left.+\mu\left(\nabla_{\boldsymbol{R}} \times \boldsymbol{K}\right) \times \hat{\boldsymbol{b}}\right] \cdot\left(\boldsymbol{v}_{\nabla B}+\boldsymbol{v}_{E, \sigma}^{(0)}\right) \partial_{u} F_{\sigma 0} \\
& +\frac{Z_{\sigma} \lambda_{\sigma}}{B}\left[\nabla_{\boldsymbol{R}} \cdot\left(\hat{\boldsymbol{b}} \times \nabla_{\boldsymbol{R}_{\perp} / \epsilon_{\sigma}}\left\langle\phi_{\sigma 1}^{\mathrm{sw}}\right\rangle F_{\sigma 1}^{\mathrm{sw}}\right)\right]^{\mathrm{lw}} \\
& -Z_{\sigma} \lambda_{\sigma} \partial_{u}\left[\left(\hat{\boldsymbol{b}} \cdot \nabla_{\boldsymbol{R}}\left\langle\phi_{\sigma 1}^{\mathrm{sw}}\right\rangle\right.\right. \\
& \left.\left.+\frac{u}{B}(\hat{\boldsymbol{b}} \times \boldsymbol{\kappa}) \cdot \nabla_{\boldsymbol{R}_{\perp} / \epsilon_{\sigma}}\left\langle\phi_{\sigma 1}^{\mathrm{sw}}\right\rangle\right) F_{\sigma 1}^{\mathrm{sw}}\right]^{\mathrm{lw}} \\
& -\frac{1}{B} \nabla_{\boldsymbol{R}} \cdot\left[Z_{\sigma} \lambda_{\sigma} \varphi_{1}^{\mathrm{l} \mathrm{w}} \hat{\boldsymbol{b}} \times \nabla_{\boldsymbol{R}} \psi\left(\Upsilon_{\sigma}+\frac{Z_{\sigma}}{T_{\sigma}} \partial_{\psi} \varphi_{0}\right) F_{\sigma 0}\right] \\
& +\frac{1}{B} \partial_{u}\left[\frac { Z _ { \sigma } \lambda _ { \sigma } \varphi _ { 1 } ^ { \mathrm { lw } } } { u } \mu ( \hat { \boldsymbol { b } } \times \nabla _ { \boldsymbol { R } } \psi ) \cdot \nabla _ { \boldsymbol { R } } B \left(\Upsilon_{\sigma}\right.\right. \\
& \left.\left.+\frac{Z_{\sigma}}{T_{\sigma}} \partial_{\psi} \varphi_{0}\right) F_{\sigma 0}\right]=\partial_{u}\left[-\frac{Z_{\sigma} \lambda_{\sigma} \varphi_{1}^{\mathrm{lw}}}{u} \sum_{\sigma^{\prime}}\left\langle\mathcal{T}_{\sigma, 0}^{*} C_{\sigma \sigma^{\prime}}^{(1) \mathrm{lw}}\right\rangle\right] \\
& +\sum_{\sigma^{\prime}}\left\langle\left[\mathcal{T}_{\sigma, 1}^{*} C_{\sigma \sigma^{\prime}}^{(1)}\right]^{\mathrm{lw}}\right\rangle+\sum_{\sigma^{\prime}}\left\langle\mathcal{T}_{\sigma, 0}^{*} C_{\sigma \sigma^{\prime}}^{(2) \mathrm{l}}\right\rangle,
\end{aligned}
$$

where we have used the relations

$$
\begin{aligned}
& Z_{\sigma} \lambda_{\sigma} \hat{\boldsymbol{b}} \cdot \nabla_{\boldsymbol{R}} \varphi_{1}^{\mathrm{lw}} \partial_{u}\left(\frac{Z_{\sigma} \lambda_{\sigma} \varphi_{1}^{\mathrm{lw}}}{T_{\sigma}} F_{\sigma 0}\right) \\
& \quad=\left(u \hat{\boldsymbol{b}} \cdot \nabla_{\boldsymbol{R}}-\mu \hat{\boldsymbol{b}} \cdot \nabla_{\boldsymbol{R}} B \partial_{u}\right)\left[-\frac{1}{2}\left(\frac{Z_{\sigma} \lambda_{\sigma} \varphi_{1}^{\mathrm{lw}}}{T_{\sigma}}\right)^{2} F_{\sigma 0}\right],
\end{aligned}
$$

$$
\begin{aligned}
& -Z_{\sigma} \lambda_{\sigma} \hat{\boldsymbol{b}} \cdot \nabla_{\boldsymbol{R}} \varphi_{1}^{\mathrm{lw}} \partial_{u} G_{\sigma 1}^{\mathrm{lw}} \\
& =\left(u \hat{\boldsymbol{b}} \cdot \nabla_{\boldsymbol{R}}-\mu \hat{\boldsymbol{b}} \cdot \nabla_{\boldsymbol{R}} B \partial_{u}\right)\left[-\frac{Z_{\sigma} \lambda_{\sigma} \varphi_{1}^{\mathrm{lw}}}{u} \partial_{u} G_{\sigma 1}^{\mathrm{lw}}\right] \\
& \quad+\partial_{u}\left[\frac{Z_{\sigma} \lambda_{\sigma} \varphi_{1}^{\mathrm{lw}}}{u}\left(u \hat{\boldsymbol{b}} \cdot \nabla_{\boldsymbol{R}}-\mu \hat{\boldsymbol{b}} \cdot \nabla_{\boldsymbol{R}} B \partial_{u}\right) G_{\sigma 1}^{\mathrm{lw}}\right], \\
& \left(\boldsymbol{v}_{M}+\boldsymbol{v}_{E, \sigma}^{(0)}\right) \cdot \nabla_{\boldsymbol{R}}\left(-\frac{Z_{\sigma} \lambda_{\sigma} \varphi_{1}^{\mathrm{lw}}}{T_{\sigma}} F_{\sigma 0}\right) \\
& -\frac{u}{B}(\hat{\boldsymbol{b}} \times \boldsymbol{\kappa}) \cdot\left(\mu \nabla_{\boldsymbol{R}} B+Z_{\sigma} \nabla_{\boldsymbol{R}} \varphi_{0}\right) \partial_{u}\left(-\frac{Z_{\sigma} \lambda_{\sigma} \varphi_{1}^{\mathrm{lw}}}{T_{\sigma}} F_{\sigma 0}\right) \\
& +\boldsymbol{v}_{E, \sigma}^{(1)} \cdot \nabla_{\boldsymbol{R}} F_{\sigma 0}-Z_{\sigma} \lambda_{\sigma} \frac{u}{B}(\hat{\boldsymbol{b}} \times \boldsymbol{\kappa}) \cdot \nabla_{\boldsymbol{R}} \varphi_{1}^{\mathrm{lw}} \partial_{u} F_{\sigma 0} \\
& =-\frac{1}{B} \nabla_{\boldsymbol{R}} \cdot\left[Z_{\sigma} \lambda_{\sigma} \varphi_{1}^{\mathrm{lw}} \hat{\boldsymbol{b}} \times \nabla_{\boldsymbol{R}} \psi\left(\Upsilon_{\sigma}+\frac{Z_{\sigma}}{T_{\sigma}} \partial_{\psi} \varphi_{0}\right) F_{\sigma 0}\right]
\end{aligned}
$$

To obtain (D.6) we have employed equation (73). Using (B.6), the relation

$$
\begin{aligned}
\left(\boldsymbol{v}_{M}+\boldsymbol{v}_{E, \sigma}^{(0)}\right) \cdot \nabla_{\boldsymbol{R}} G_{\sigma 1}^{\mathrm{lw}}+u \boldsymbol{\kappa} \cdot\left(\boldsymbol{v}_{\nabla B}+\boldsymbol{v}_{E, \sigma}^{(0)}\right) \partial_{u} G_{\sigma 1}^{\mathrm{lw}} & =\frac{1}{B} \nabla_{\boldsymbol{R}} \cdot\left[\left(\mu \hat{\boldsymbol{b}} \times \nabla_{\boldsymbol{R}} B+u^{2} \nabla_{\boldsymbol{R}} \times \hat{\boldsymbol{b}}+Z_{\sigma} \hat{\boldsymbol{b}} \times \nabla_{\boldsymbol{R}} \varphi_{0}\right) G_{\sigma 1}^{\mathrm{lw}}\right] \\
& -\frac{1}{B} \partial_{u}\left[u\left(\nabla_{\boldsymbol{R}} \times \hat{\boldsymbol{b}}\right) \cdot\left(\mu \nabla_{\boldsymbol{R}} B+Z_{\sigma} \nabla_{\boldsymbol{R}} \varphi_{0}\right) G_{\sigma 1}^{\mathrm{lw}}\right] \\
& -\frac{u}{B} \hat{\boldsymbol{b}} \cdot \nabla_{\boldsymbol{R}} \times \hat{\boldsymbol{b}}\left(u \hat{\boldsymbol{b}} \cdot \nabla_{\boldsymbol{R}}-\mu \hat{\boldsymbol{b}} \cdot \nabla_{\boldsymbol{R}} B \partial_{u}\right) G_{\sigma 1}^{\mathrm{lw}}
\end{aligned}
$$

and

and again (73) to write

$$
\begin{aligned}
& -\frac{u}{B}\left(\hat{\boldsymbol{b}} \cdot \nabla_{\boldsymbol{R}} \times \hat{\boldsymbol{b}}\right)\left[\left(u \hat{\boldsymbol{b}} \cdot \nabla_{\boldsymbol{R}}-\mu \hat{\boldsymbol{b}} \cdot \nabla_{\boldsymbol{R}} B \partial_{u}\right) G_{\sigma 1}^{\mathrm{lw}}\right. \\
& \left.\quad+\left(\boldsymbol{v}_{M}+\boldsymbol{v}_{E, \sigma}^{(0)}\right) \cdot \nabla_{\boldsymbol{R}} F_{\sigma 0}+u \boldsymbol{\kappa} \cdot\left(\boldsymbol{v}_{\nabla B}+\boldsymbol{v}_{E, \sigma}^{(0)}\right) \partial_{u} F_{\sigma 0}\right] \\
& =-\frac{u}{B}\left(\hat{\boldsymbol{b}} \cdot \nabla_{\boldsymbol{R}} \times \hat{\boldsymbol{b}}\right) \sum_{\sigma^{\prime}}\left\langle\mathcal{T}_{\sigma, 0}^{*} C_{\sigma \sigma^{\prime}}^{(1) 1 \mathrm{w}}\right\rangle,
\end{aligned}
$$

we find

$\left(u \hat{\boldsymbol{b}} \cdot \nabla_{\boldsymbol{R}}-\mu \hat{\boldsymbol{b}} \cdot \nabla_{\boldsymbol{R}} B \partial_{u}\right) G_{\sigma 2}^{\mathrm{lw}}+\frac{\lambda_{\sigma}^{2}}{\tau_{\sigma}} \partial_{\epsilon_{\mathrm{s}}^{2} t} F_{\sigma 0}$

$\frac{1}{B} \nabla_{\boldsymbol{R}} \cdot\left[\left(\mu \hat{\boldsymbol{b}} \times \nabla_{\boldsymbol{R}} B+u^{2} \nabla_{\boldsymbol{R}} \times \hat{\boldsymbol{b}}+Z_{\sigma} \hat{\boldsymbol{b}} \times \nabla_{\boldsymbol{R}} \varphi_{0}\right) G_{\sigma 1}^{\mathrm{lw}}\right.$

$\left.-Z_{\sigma} \lambda_{\sigma} \varphi_{1}^{\mathrm{lw}} \hat{\boldsymbol{b}} \times \nabla_{\boldsymbol{R}} \psi\left(\Upsilon_{\sigma}+\frac{Z_{\sigma}}{T_{\sigma}} \partial_{\psi} \varphi_{0}\right) F_{\sigma 0}\right]$

$-\frac{1}{B} \partial_{u}\left[u\left(\nabla_{\boldsymbol{R}} \times \hat{\boldsymbol{b}}\right) \cdot\left(\mu \nabla_{\boldsymbol{R}} B+Z_{\sigma} \nabla_{\boldsymbol{R}} \varphi_{0}\right) G_{\sigma 1}^{\mathrm{lw}}\right.$

$\left.-\frac{Z_{\sigma} \lambda_{\sigma} \varphi_{1}^{\mathrm{lw}}}{u} \mu\left(\hat{\boldsymbol{b}} \times \nabla_{\boldsymbol{R}} \psi\right) \cdot \nabla_{\boldsymbol{R}} B\left(\Upsilon_{\sigma}+\frac{Z_{\sigma}}{T_{\sigma}} \partial_{\psi} \varphi_{0}\right) F_{\sigma 0}\right]$

$-\frac{u \mu}{B}\left(\nabla_{\boldsymbol{R}} \times \boldsymbol{K}\right)_{\perp} \cdot \nabla_{\boldsymbol{R}} F_{\sigma 0}$

$+\mu\left(\nabla_{\boldsymbol{R}} \times \boldsymbol{K}\right)_{\perp} \cdot\left(\mu \nabla_{\boldsymbol{R}} B+Z_{\sigma} \nabla_{\boldsymbol{R}} \varphi_{0}\right) \partial_{u} F_{\sigma 0}$ 


$$
\begin{aligned}
& +\frac{Z_{\sigma} \lambda_{\sigma}}{B}\left[\nabla_{\boldsymbol{R}} \cdot\left(\hat{\boldsymbol{b}} \times \nabla_{\boldsymbol{R}_{\perp} / \epsilon_{\sigma}}\left\langle\phi_{\sigma 1}^{\mathrm{sw}}\right\rangle F_{\sigma 1}^{\mathrm{sw}}\right)\right]^{\mathrm{lw}} \\
& -Z_{\sigma} \lambda_{\sigma} \partial_{u}\left[\left(\hat{\boldsymbol{b}} \cdot \nabla_{\boldsymbol{R}}\left\langle\phi_{\sigma 1}^{\mathrm{sw}}\right\rangle+\frac{u}{B}(\hat{\boldsymbol{b}} \times \boldsymbol{\kappa}) \cdot \nabla_{\boldsymbol{R}_{\perp} / \epsilon_{\sigma}}\left\langle\phi_{\sigma 1}^{\mathrm{sw}}\right\rangle\right) F_{\sigma 1}^{\mathrm{sw}}\right]^{\mathrm{lw}} \\
& =\partial_{u}\left[-\frac{Z_{\sigma} \lambda_{\sigma} \varphi_{1}^{1 \mathrm{w}}}{u} \sum_{\sigma^{\prime}}\left\langle\mathcal{T}_{\sigma, 0}^{*} C_{\sigma \sigma^{\prime}}^{(1) \mathrm{lw}}\right\rangle\right] \\
& +\partial_{u}\left(\left\langle u \hat{\boldsymbol{b}} \cdot \nabla_{\boldsymbol{R}} \hat{\boldsymbol{b}} \cdot \boldsymbol{\rho} \mathcal{T}_{\sigma, 0}^{*} C_{\sigma \sigma^{\prime}}^{(1) \mathrm{lw}}\right\rangle\right. \\
& \left.-\mu \hat{\boldsymbol{b}} \cdot \nabla_{\boldsymbol{R}} \times \hat{\boldsymbol{b}}\left\langle\mathcal{T}_{\sigma, 0}^{*} C_{\sigma \sigma^{\prime}}^{(1) \mathrm{lw}}\right\rangle\right) \\
& +\partial_{\mu}\left\{\frac{u \mu}{B} \hat{\boldsymbol{b}} \cdot \nabla_{\boldsymbol{R}} \times \hat{\boldsymbol{b}}\left\langle\mathcal{T}_{\sigma, 0}^{*} C_{\sigma \sigma^{\prime}}^{(1) \mathrm{w}}\right\rangle\right. \\
& -\left\langle\left(\frac{Z_{\sigma}}{B} \rho \cdot \nabla_{R} \varphi_{0}+\frac{\mu}{B} \rho \cdot \nabla_{R} B\right.\right. \\
& \left.\left.\left.+\frac{u^{2}}{B} \hat{\boldsymbol{b}} \cdot \nabla \hat{\boldsymbol{b}} \cdot \boldsymbol{\rho}\right) \mathcal{T}_{\sigma, 0}^{*} C_{\sigma \sigma^{\prime}}^{(1) \mathrm{lw}}\right\rangle\right\}+\frac{1}{B} \nabla_{\boldsymbol{R}} \cdot\left\langle B \boldsymbol{\rho} \mathcal{T}_{\sigma, 0}^{*} C_{\sigma \sigma^{\prime}}^{(1) \mathrm{lw}}\right\rangle \\
& +\left\langle\left[\mathcal{T}_{\sigma, 1}^{*} C_{\sigma \sigma^{\prime}}^{(1) \mathrm{sw}}\right]^{1 \mathrm{w}}\right\rangle+\sum_{\sigma^{\prime}}\left\langle\mathcal{T}_{\sigma, 0}^{*} C_{\sigma \sigma^{\prime}}^{(2) \mathrm{lw}}\right\rangle \text {. }
\end{aligned}
$$

Finally, we remind the reader that the term $\left\langle\left[\mathcal{T}_{\sigma, 1}^{*} C_{\sigma \sigma^{\prime}}^{(1) \mathrm{sw}}\right]^{\mathrm{lw}}\right\rangle$ is a total derivative with respect to $\mu$ (see (B.7)).

\section{Appendix E. Calculation of the particle density transport equation}

The transport equation for the particle density of species $\sigma$ is obtained from (100), with $j=2$ and $R_{\sigma 2}$ given by (99). Easily, one gets

$$
\begin{gathered}
\partial_{\epsilon_{\mathrm{s}}^{2}} n_{\sigma}=\left\langle\frac { \tau _ { \sigma } } { \lambda _ { \sigma } ^ { 2 } } \int \left\{-\nabla_{\boldsymbol{R}} \cdot\left[\left(\mu \hat{\boldsymbol{b}} \times \nabla_{\boldsymbol{R}} B\right.\right.\right.\right. \\
\left.+u^{2} \nabla_{\boldsymbol{R}} \times \hat{\boldsymbol{b}}+Z_{\sigma} \hat{\boldsymbol{b}} \times \nabla_{\boldsymbol{R}} \varphi_{0}\right) G_{\sigma 1}^{\mathrm{lw}} \\
\left.-Z_{\sigma} \lambda_{\sigma} \varphi_{1}^{\mathrm{lw}} \hat{\boldsymbol{b}} \times \nabla_{\boldsymbol{R}} \psi\left(\Upsilon_{\sigma}+\frac{Z_{\sigma}}{T_{\sigma}} \partial_{\psi} \varphi_{0}\right) F_{\sigma 0}\right] \\
-Z_{\sigma} \lambda_{\sigma}\left[\nabla_{\boldsymbol{R}} \cdot\left(\hat{\boldsymbol{b}} \times \nabla_{\boldsymbol{R}_{\perp} / \epsilon_{\sigma}}\left\langle\phi_{\sigma 1}^{\mathrm{sw}}\right\rangle F_{\sigma 1}^{\mathrm{sw}}\right)\right]^{\mathrm{lw}} \\
\left.\left.+\sum_{\sigma^{\prime}} \nabla_{\boldsymbol{R}} \cdot\left\langle B \boldsymbol{\rho} \mathcal{T}_{\sigma, 0}^{*} C_{\sigma \sigma^{\prime}}^{(1) 1 \mathrm{w}}\right\rangle\right\} \mathrm{d} u \mathrm{~d} \mu \mathrm{d} \theta\right\rangle_{\psi},
\end{gathered}
$$

where the particle conservation property of the collision operator, integrations by parts in $u$ and arguments of parity in $u$ have been used to drop many terms from $R_{\sigma 2}$. Also, we have used the fact that the term $\left\langle\left[\mathcal{T}_{\sigma, 1}^{*} C_{\sigma \sigma^{\prime}}^{(1) \mathrm{sw}}\right]^{\mathrm{lw}}\right\rangle$ is an exact derivative in $\mu$, as shown in (B.7).

Finally, we recall (106) and the fact that $\varphi_{0}$ is a flux function to obtain (107).

\section{Appendix F. Calculation of the energy transport equation}

It is illustrative to show that the transport equation for the energy density of species $\sigma$ is not a solvability condition, because it still involves $\left\langle F_{\sigma 2}^{1 \mathrm{w}}\right\rangle$. It is obtained by multiplying (D.9) by $\tau_{\sigma} \lambda_{\sigma}^{-2} B\left(u^{2} / 2+\mu B\right)$, integrating over $u, \mu$ and $\theta$, and taking the flux-surface average, giving

$$
\begin{aligned}
& \partial_{\epsilon_{\mathrm{s}}^{2} t}\left(\frac{3}{2} n_{\sigma} T_{\sigma}\right) \\
& +\left\langle\nabla _ { \boldsymbol { R } } \cdot \int \frac { \tau _ { \sigma } } { \lambda _ { \sigma } ^ { 2 } } B ( u ^ { 2 } / 2 + \mu B ) \left[\left(\boldsymbol{v}_{\nabla B}+\boldsymbol{v}_{E, \sigma}^{(0)}\right) G_{\sigma 1}^{\mathrm{lw}}\right.\right. \\
& \left.\left.-Z_{\sigma} \lambda_{\sigma} \varphi_{1}^{\mathrm{l} w} \hat{\boldsymbol{b}} \times \nabla_{\boldsymbol{R}} \psi\left(\Upsilon_{\sigma}+\frac{Z_{\sigma}}{T_{\sigma}} \partial_{\psi} \varphi_{0}\right) F_{\sigma 0}\right] \mathrm{~d} u \mathrm{~d} \mu \mathrm{d} \theta\right\rangle_{\psi} \\
& +\left\langle\nabla_{\boldsymbol{R}} \cdot \int \frac{\tau_{\sigma}}{\lambda_{\sigma}^{2}} u^{2}\left(u^{2} / 2+\mu B\right) \nabla_{\boldsymbol{R}} \times \hat{\boldsymbol{b}} G_{\sigma 1}^{\mathrm{lw}} \mathrm{d} u \mathrm{~d} \mu \mathrm{d} \theta\right\rangle_{\psi} \\
& +\left\langle\nabla _ { \boldsymbol { R } } \cdot \int ( u ^ { 2 } / 2 + \mu B ) \left\{\left[\left(\hat{\boldsymbol{b}} \times \nabla_{\boldsymbol{R}_{\perp} / \epsilon_{\sigma}}\left\langle\phi_{\sigma 1}^{\mathrm{sw}}\right\rangle F_{\sigma 1}^{\mathrm{sw}}\right)\right]^{\mathrm{lw}}\right.\right. \\
& \left.\left.-\frac{\tau_{\sigma}}{\lambda_{\sigma}^{2}} \sum_{\sigma^{\prime}}\left\langle B \rho \mathcal{T}_{\sigma, 0}^{*} C_{\sigma \sigma^{\prime}}^{(1) l \mathrm{w}}\right\rangle\right\} \mathrm{d} u \mathrm{~d} \mu \mathrm{d} \theta\right\rangle_{\psi} \\
& +\left\langle\int B \boldsymbol{v}_{M} \cdot\left[\nabla_{\boldsymbol{R}_{\perp} / \epsilon_{\sigma}}\left\langle\phi_{\sigma 1}^{\mathrm{sw}}\right\rangle F_{\sigma 1}^{\mathrm{sw}}\right]^{\mathrm{lw}} \mathrm{d} u \mathrm{~d} \mu \mathrm{d} \theta\right\rangle_{\psi} \\
& +\left\langle\int B u\left[\hat{b} \cdot \nabla_{R}\left\langle\phi_{\sigma 1}^{\mathrm{sw}}\right\rangle F_{\sigma 1}^{\mathrm{sw}}\right]^{\mathrm{lw}} \mathrm{d} u \mathrm{~d} \mu \mathrm{d} \theta\right\rangle_{\psi} \\
& +\frac{1}{\lambda_{\sigma}}\left\langle\int B \boldsymbol{v}_{M} \cdot \nabla_{\boldsymbol{R}} \varphi_{0} G_{\sigma 1}^{\mathrm{lw}} \mathrm{d} u \mathrm{~d} \mu \mathrm{d} \theta\right\rangle_{\psi} \\
& =\left\langle\frac{\tau_{\sigma}}{\lambda_{\sigma}^{2}} \int B\left(u^{2} / 2+\mu B\right) \sum_{\sigma^{\prime}}\left\langle\left[\mathcal{T}_{\sigma, 1}^{*} C_{\sigma \sigma^{\prime}}^{(1) \mathrm{sw}}\right]^{1 \mathrm{lw}}\right\rangle \mathrm{d} u \mathrm{~d} \mu \mathrm{d} \theta\right\rangle_{\psi} \\
& +\left\langle\frac{\tau_{\sigma}}{\lambda_{\sigma}^{2}} \int B Z_{\sigma} \sum_{\sigma^{\prime}}\left\langle\rho \cdot \nabla_{R} \varphi_{0} \mathcal{T}_{\sigma, 0}^{*} C_{\sigma \sigma^{\prime}}^{(1) \mathrm{lw}}\right\rangle \mathrm{d} u \mathrm{~d} \mu \mathrm{d} \theta\right\rangle_{\psi} \\
& +\left\langle\frac{\tau_{\sigma}}{\lambda_{\sigma}^{2}} \int B\left(u^{2} / 2+\mu B\right) \sum_{\sigma^{\prime}}\left\langle\mathcal{T}_{\sigma, 0}^{*} C_{\sigma \sigma^{\prime}}^{(2) l \mathrm{w}}\right\rangle \mathrm{d} u \mathrm{~d} \mu \mathrm{d} \theta\right\rangle_{\psi} .
\end{aligned}
$$

Here, conservation of particles by the collision operator, integrations by parts in $\boldsymbol{R}, u$ and $\mu$, and arguments of parity in $u$ have been employed. The last term of (F.1) still contains $\left\langle F_{\sigma 2}^{\mathrm{lw}}\right\rangle$. Summing (F.1) over $\sigma$, one gets the solvability condition corresponding to (101) for $j=2$ and $R_{\sigma 2}$ given in (99), which is a transport equation for the total energy. Hence, adding (F.1) over all the species:

$$
\begin{aligned}
\partial_{\epsilon_{\mathrm{s}}^{2} t} & \sum_{\sigma} \frac{3}{2} n_{\sigma} T_{\sigma} \\
& +\left\langle\nabla _ { \boldsymbol { R } } \cdot \sum _ { \sigma } \frac { \tau _ { \sigma } } { \lambda _ { \sigma } ^ { 2 } } \int B ( u ^ { 2 } / 2 + \mu B ) \left[\left(\boldsymbol{v}_{\nabla B}\right.\right.\right.
\end{aligned}
$$




$$
\begin{aligned}
& \left.+\boldsymbol{v}_{E, \sigma}^{(0)}+u^{2} \nabla_{\boldsymbol{R}} \times \hat{\boldsymbol{b}}\right) G_{\sigma 1}^{\mathrm{lw}} \\
& \left.\left.-Z_{\sigma} \lambda_{\sigma} \varphi_{1}^{\mathrm{lw}} \hat{\boldsymbol{b}} \times \nabla_{\boldsymbol{R}} \psi\left(\Upsilon_{\sigma}+\frac{Z_{\sigma}}{T_{\sigma}} \partial_{\psi} \varphi_{0}\right) F_{\sigma 0}\right] \mathrm{~d} u \mathrm{~d} \mu \mathrm{d} \theta\right\rangle_{\psi} \\
& +\left\langle\nabla _ { \boldsymbol { R } } \cdot \int ( u ^ { 2 } / 2 + \mu B ) \left\{\sum_{\sigma}\left[\left(\hat{\boldsymbol{b}} \times \nabla_{\boldsymbol{R}_{\perp} / \epsilon_{\sigma}}\left\langle\phi_{\sigma 1}^{\mathrm{sw}}\right\rangle F_{\sigma 1}^{\mathrm{sw}}\right)\right]^{\mathrm{lw}}\right.\right. \\
& \left.\left.-\sum_{\sigma, \sigma^{\prime}} \frac{\tau_{\sigma}}{\lambda_{\sigma}^{2}}\left\langle B \rho \mathcal{T}_{\sigma, 0}^{*} C_{\sigma \sigma^{\prime}}^{(1) \mathrm{lw}}\right\rangle\right\} \mathrm{d} u \mathrm{~d} \mu \mathrm{d} \theta\right\rangle_{\psi} \\
& +\sum_{\sigma}\left\langle\int B \boldsymbol{v}_{M} \cdot\left[\nabla_{\boldsymbol{R}_{\perp} / \epsilon_{\sigma}}\left\langle\phi_{\sigma 1}^{\mathrm{sw}}\right\rangle F_{\sigma 1}^{\mathrm{sw}}\right]^{\mathrm{lw}} \mathrm{d} u \mathrm{~d} \mu \mathrm{d} \theta\right\rangle_{\psi} \\
& +\sum_{\sigma}\left\langle\int B u\left[\hat{\boldsymbol{b}} \cdot \nabla_{\boldsymbol{R}}\left\langle\phi_{\sigma 1}^{\mathrm{sw}}\right\rangle F_{\sigma 1}^{\mathrm{sw}}\right]^{\mathrm{lw}} \mathrm{d} u \mathrm{~d} \mu \mathrm{d} \theta\right\rangle_{\psi} \\
& -\left\langle\int B\left(u^{2} / 2+\mu B\right) \sum_{\sigma, \sigma^{\prime}} \frac{\tau_{\sigma}}{\lambda_{\sigma}^{2}}\left\langle\left[\mathcal{T}_{\sigma, 1}^{*} C_{\sigma \sigma^{\prime}}^{(1) \mathrm{sw}}\right]^{1 \mathrm{w}}\right\rangle \mathrm{d} u \mathrm{~d} \mu \mathrm{d} \theta\right\rangle_{\psi} \\
& +\partial_{\psi} \varphi_{0} \sum_{\sigma} \frac{1}{\lambda_{\sigma}}\left\langle\int B \boldsymbol{v}_{M} \cdot \nabla_{\boldsymbol{R}} \psi G_{\sigma 1}^{\mathrm{lw}} \mathrm{d} u \mathrm{~d} \mu \mathrm{d} \theta\right\rangle_{\psi}=0 .
\end{aligned}
$$

We have employed the momentum and energy conserving properties of the collision operator to deduce that

$$
\begin{aligned}
& \sum_{\sigma, \sigma^{\prime}}\left\langle\frac{1}{\lambda_{\sigma}} \int B\left\langle\rho \cdot \nabla_{R} \psi \mathcal{T}_{\sigma, 0}^{*} C_{\sigma \sigma^{\prime}}^{(1) \mathrm{lw}}\right\rangle \mathrm{d} u \mathrm{~d} \mu \mathrm{d} \theta\right\rangle_{\psi}=0, \\
& \sum_{\sigma, \sigma^{\prime}}\left\langle\frac{\tau_{\sigma}}{\lambda_{\sigma}^{2}} \int B\left(u^{2} / 2+\mu B\right)\left\langle\mathcal{T}_{\sigma, 0}^{*} C_{\sigma \sigma^{\prime}}^{(2) \mathrm{l}}\right\rangle \mathrm{d} u \mathrm{~d} \mu \mathrm{d} \theta\right\rangle_{\psi}=0 .
\end{aligned}
$$

The vanishing of these terms is checked easily after realizing that the sums over $\sigma$ and $\sigma^{\prime}$ can be split into terms of the form

$$
\begin{aligned}
& \int B \boldsymbol{\rho} \cdot \nabla_{\boldsymbol{R}} \psi\left(\mathcal{T}_{\sigma, 0}^{*} C_{\sigma \sigma^{\prime}}\left[\lambda_{\sigma}^{-1} g_{\sigma}, \mathcal{T}_{\sigma^{\prime}, 0}^{-1 *} F_{\sigma^{\prime} 0}\right]\right. \\
& \left.+\mathcal{T}_{\sigma^{\prime}, 0}^{*} C_{\sigma^{\prime} \sigma}\left[\mathcal{T}_{\sigma^{\prime}, 0}^{-1 *} F_{\sigma^{\prime} 0}, \lambda_{\sigma}^{-1} g_{\sigma}\right]\right) \mathrm{d} u \mathrm{~d} \mu \mathrm{d} \theta=0, \\
& \int B\left(u^{2} / 2+\mu B\right)\left(\tau_{\sigma} \mathcal{T}_{\sigma, 0}^{*} C_{\sigma \sigma^{\prime}}\left[\lambda_{\sigma}^{-1} g_{\sigma}, \lambda_{\sigma^{\prime}}^{-1} g_{\sigma^{\prime}}\right]\right. \\
& \left.+\tau_{\sigma^{\prime}} \mathcal{T}_{\sigma^{\prime}, 0}^{*} C_{\sigma^{\prime} \sigma}\left[\lambda_{\sigma^{\prime}}^{-1} g_{\sigma^{\prime}}, \lambda_{\sigma}^{-1} g_{\sigma}\right]\right) \mathrm{d} u \mathrm{~d} \mu \mathrm{d} \theta=0, \\
& \int B\left(u^{2} / 2+\mu B\right)\left(\tau_{\sigma} \mathcal{T}_{\sigma, 0}^{*} C_{\sigma \sigma^{\prime}}\left[\lambda_{\sigma}^{-2} g_{\sigma}, \mathcal{T}_{\sigma^{\prime}, 0}^{-1 *} F_{\sigma^{\prime} 0}\right]\right. \\
& \left.+\tau_{\sigma^{\prime}} \mathcal{T}_{\sigma^{\prime}, 0}^{*} C_{\sigma^{\prime} \sigma}\left[\mathcal{T}_{\sigma^{\prime}, 0}^{-1 *} F_{\sigma^{\prime} 0}, \lambda_{\sigma}^{-2} g_{\sigma}\right]\right) \mathrm{d} u \mathrm{~d} \mu \mathrm{d} \theta=0,
\end{aligned}
$$

that are zero because of (A.9) for any pair of functions $g_{\sigma}(\boldsymbol{r}, \boldsymbol{v}, t)$ and $g_{\sigma^{\prime}}(\boldsymbol{r}, \boldsymbol{v}, t)$.

In appendix L of [14] it has been shown that, independently of the magnetic geometry,

$$
\begin{aligned}
& \left\langle\sum _ { \sigma } \int B \left[F _ { \sigma 1 } ^ { \mathrm { sw } } \left( u \hat{\boldsymbol{b}} \cdot \nabla_{\boldsymbol{R}}\left\langle\phi_{\sigma 1}^{\mathrm{sw}}\right\rangle\right.\right.\right. \\
& \left.\left.\left.\quad+\boldsymbol{v}_{M} \cdot \nabla_{\boldsymbol{R}_{\perp} / \epsilon_{\sigma}}\left\langle\phi_{\sigma 1}^{\mathrm{sw}}\right\rangle\right)\right]^{\mathrm{lw}} \mathrm{d} u \mathrm{~d} \mu \mathrm{d} \theta\right\rangle_{\psi}
\end{aligned}
$$

$$
\begin{aligned}
& -\left\langle\sum_{\sigma, \sigma^{\prime}} \frac{\tau_{\sigma}}{\lambda_{\sigma}^{2}} \int B\left(u^{2} / 2+\mu B\right)\left[\left\langle\mathcal{T}_{\sigma, 1}^{*} C_{\sigma \sigma^{\prime}}^{(1) \mathrm{sw}}\right\rangle\right]^{\mathrm{lw}} \mathrm{d} u \mathrm{~d} \mu \mathrm{d} \theta\right\rangle_{\psi} \\
& =O\left(\epsilon_{\mathrm{s}}\right) .
\end{aligned}
$$

Besides, since $\varphi_{0}$ is a flux function, the ambipolarity condition (114) makes the last term on the left side of (F.2) vanish. Then, we can drop the terms that are not written explicitly as a divergence in (F.2). Using formula (106) to rewrite the remaining ones, we reach (108).

\section{Appendix G. Collision operator in drift-kinetic coordinates}

For the proof of section 7 it is useful to exhibit the form of the kernel of the collision operator in coordinates $(\boldsymbol{R}, u, \mu, \theta)=$ $\mathcal{T}_{\sigma, 0}^{-1}(\boldsymbol{r}, \boldsymbol{v})$. Recall that when $\epsilon_{\sigma}=0$ the change of coordinates is simply given by

$$
\begin{gathered}
\boldsymbol{R}=\boldsymbol{r}, \\
u=\boldsymbol{v} \cdot \hat{\boldsymbol{b}}(\boldsymbol{r}), \\
\mu=\frac{1}{2 B(\boldsymbol{r})}(\boldsymbol{v}-\boldsymbol{v} \cdot \hat{\boldsymbol{b}}(\boldsymbol{r}) \hat{\boldsymbol{b}}(\boldsymbol{r}))^{2}, \\
\theta=\arctan \left(\frac{\boldsymbol{v} \cdot \hat{\boldsymbol{e}}_{2}(\boldsymbol{r})}{\boldsymbol{v} \cdot \hat{\boldsymbol{e}}_{1}(\boldsymbol{r})}\right) .
\end{gathered}
$$

Since $\boldsymbol{R}=\boldsymbol{r}$, to this order the change of coordinates only affects the velocities and the spatial position enters as a parameter. An additional simplification is provided by the fact that, for the purposes of section 7 , we can restrict ourselves to gyrophase-independent distribution functions.

It is convenient to rewrite the collision operator (4) in terms of the Rosenbluth potentials $p_{\sigma^{\prime}}(\boldsymbol{r}, \boldsymbol{v}, t)$ and $q_{\sigma^{\prime}}(\boldsymbol{r}, \boldsymbol{v}, t)$, defined by

$$
p_{\sigma^{\prime}}=\int_{\mathbb{R}^{3}}\left|\boldsymbol{v}-\boldsymbol{v}^{\prime}\right| f_{\sigma^{\prime}}\left(\boldsymbol{r}, \boldsymbol{v}^{\prime}, t\right) \mathrm{d}^{3} v^{\prime}
$$

and

$$
q_{\sigma^{\prime}}=\int_{\mathbb{R}^{3}} \frac{2}{\left|\boldsymbol{v}-\boldsymbol{v}^{\prime}\right|} f_{\sigma^{\prime}}\left(\boldsymbol{r}, \boldsymbol{v}^{\prime}, t\right) \mathrm{d}^{3} v^{\prime} .
$$

Namely,

$$
\begin{aligned}
C_{\sigma \sigma^{\prime}} & {\left[f_{\sigma}, f_{\sigma^{\prime}}\right](\boldsymbol{r}, \boldsymbol{v}) } \\
= & \frac{\gamma_{\sigma \sigma^{\prime}}}{m_{\sigma}} \nabla_{\boldsymbol{v}} \cdot\left(\frac{1}{m_{\sigma}} \nabla_{\boldsymbol{v}} \nabla_{\boldsymbol{v}} p_{\sigma^{\prime}}(\boldsymbol{r}, \boldsymbol{v}, t) \cdot \nabla_{\boldsymbol{v}} f_{\sigma}(\boldsymbol{r}, \boldsymbol{v}, t)\right. \\
& \left.-\frac{1}{m_{\sigma^{\prime}}} f_{\sigma}(\boldsymbol{r}, \boldsymbol{v}, t) \nabla_{\boldsymbol{v}} q_{\sigma^{\prime}}(\boldsymbol{r}, \boldsymbol{v}, t)\right) .
\end{aligned}
$$

This can be immediately checked by using

$$
\overleftrightarrow{\boldsymbol{W}}(\boldsymbol{w})=\nabla_{\boldsymbol{w}} \nabla_{\boldsymbol{w}} w
$$

and

$$
\nabla_{\boldsymbol{w}} \cdot \stackrel{\leftrightarrow}{\boldsymbol{W}}(\boldsymbol{w})=\nabla_{\boldsymbol{w}}\left(\frac{2}{w}\right)
$$


where $\stackrel{\leftrightarrow}{\boldsymbol{W}}(\boldsymbol{w})$ has been defined in (6). An equivalent definition of the Rosenbluth potentials is provided by saying that they are the solutions of

$$
\nabla_{v}^{2} p_{\sigma^{\prime}}(\boldsymbol{r}, \boldsymbol{v}, t)=q_{\sigma^{\prime}}(\boldsymbol{r}, \boldsymbol{v}, t)
$$

and

$$
\nabla_{v}^{2} q_{\sigma^{\prime}}(\boldsymbol{r}, \boldsymbol{v}, t)=-8 \pi f_{\sigma^{\prime}}(\boldsymbol{r}, \boldsymbol{v}, t)
$$

with the appropriate boundary conditions. In order to show that (G.2) and (G.3) fulfill these equations, one has to use

$$
\nabla_{\boldsymbol{w}}^{2} w=\frac{2}{w}
$$

and

$$
\nabla_{w}^{2}\left(\frac{1}{w}\right)=-4 \pi \delta(\boldsymbol{w}) .
$$

It is more convenient for us to take (G.7) and (G.8) as the expressions defining the potentials.

Denote by $F_{\sigma}(\boldsymbol{R}, u, \mu, t), P_{\sigma^{\prime}}(\boldsymbol{R}, u, \mu, t), Q_{\sigma^{\prime}}(\boldsymbol{R}, u, \times$ $\mu, t)$, the distribution function and Rosenbluth potentials in the new coordinates. That is,

$$
\begin{gathered}
F_{\sigma}(\boldsymbol{R}, u, \mu, t)=\mathcal{T}_{\sigma, 0}^{*} f_{\sigma}, \\
P_{\sigma^{\prime}}(\boldsymbol{R}, u, \mu, t)=\mathcal{T}_{\sigma^{\prime}, 0}^{*} p_{\sigma^{\prime}}, \\
Q_{\sigma^{\prime}}(\boldsymbol{R}, u, \mu, t)=\mathcal{T}_{\sigma^{\prime}, 0}^{*} q_{\sigma^{\prime}} .
\end{gathered}
$$

Since the distribution functions are gyrophase independent, $P_{\sigma^{\prime}}$ and $Q_{\sigma^{\prime}}$ are also required to be so. We have to apply to (G.4) the divergence formula for a transformation from euclidean coordinates $\boldsymbol{v}$ to arbitrary coordinates $\boldsymbol{Y}$ :

$$
\nabla_{\boldsymbol{v}} \cdot \boldsymbol{\Gamma}_{\sigma \sigma^{\prime}}=\sum_{i=1}^{3} \frac{1}{\mathcal{J}} \frac{\partial}{\partial Y^{i}}\left(\mathcal{J} \boldsymbol{\Gamma}_{\sigma \sigma^{\prime}} \cdot \nabla_{\boldsymbol{v}} Y^{i}\right),
$$

where $\mathcal{J}=\operatorname{det}(\partial \boldsymbol{v} / \partial \boldsymbol{Y})$. In our case, $\boldsymbol{Y} \equiv\{u, \mu, \theta\}$ and the transformation is given in (G.1) (recall that the spatial coordinates do not change, so we abuse a bit the notation and understand the transformation as acting only on velocity coordinates). The vector field $\boldsymbol{\Gamma}_{\sigma \sigma^{\prime}}$ reads

$$
\begin{aligned}
\boldsymbol{\Gamma}_{\sigma \sigma^{\prime}} & =\frac{\gamma_{\sigma \sigma^{\prime}}}{m_{\sigma}}\left(\frac{1}{m_{\sigma}} \nabla_{\boldsymbol{v}} \nabla_{\boldsymbol{v}} p_{\sigma^{\prime}}(\boldsymbol{r}, \boldsymbol{v}, t) \cdot \nabla_{\boldsymbol{v}} f_{\sigma}(\boldsymbol{r}, \boldsymbol{v}, t)\right. \\
& \left.-\frac{1}{m_{\sigma^{\prime}}} f_{\sigma}(\boldsymbol{r}, \boldsymbol{v}, t) \nabla_{\boldsymbol{v}} q_{\sigma^{\prime}}(\boldsymbol{r}, \boldsymbol{v}, t)\right),
\end{aligned}
$$

$\mathcal{J}=B, \nabla_{\boldsymbol{v}} u=\hat{\boldsymbol{b}}, \nabla_{\boldsymbol{v}} \mu=B^{-1} \boldsymbol{v}_{\perp}$, and $\nabla_{\boldsymbol{v}} \theta=\left(\hat{\boldsymbol{b}} \times \boldsymbol{v}_{\perp}\right) / \boldsymbol{v}_{\perp}^{2}$. Here, $\boldsymbol{v}_{\perp}=\boldsymbol{v}-\boldsymbol{v} \cdot \hat{\boldsymbol{b}} \hat{\boldsymbol{b}}$.

We proceed to write (G.4) in the new coordinates, and denote it by $C_{\sigma \sigma^{\prime}}^{\mathcal{T}_{\sigma, 0}}\left[F_{\sigma}, F_{\sigma^{\prime}}\right]$,

$$
C_{\sigma \sigma^{\prime}}^{\mathcal{T}_{\sigma, 0}}\left[F_{\sigma}, F_{\sigma^{\prime}}\right]=\partial_{u} \Gamma_{\sigma \sigma^{\prime}}^{u}+\partial_{\mu} \Gamma_{\sigma \sigma^{\prime}}^{\mu}+\partial_{\theta} \Gamma_{\sigma \sigma^{\prime}}^{\theta} .
$$

Here, $\Gamma_{\sigma \sigma^{\prime}}^{i}:=\Gamma_{\sigma \sigma^{\prime}} \cdot \nabla_{v} Y^{i}$. Noting that $\partial_{u}\left(\nabla_{v} \mu\right)=0$, $\partial_{\mu}\left(\nabla_{v} \mu\right)=(2 B \mu)^{-1} \boldsymbol{v}_{\perp}$, it is easy to reach explicit expressions for $\Gamma_{\sigma \sigma^{\prime}}^{u}, \Gamma_{\sigma \sigma^{\prime}}^{\mu}$ and $\Gamma_{\sigma \sigma^{\prime}}^{\theta}$ :

$\Gamma_{\sigma \sigma^{\prime}}^{u}=\frac{\gamma_{\sigma \sigma^{\prime}}}{m_{\sigma}^{2}}\left(\partial_{u}^{2} P_{\sigma^{\prime}} \partial_{u} F_{\sigma}\right.$

$$
\left.+\frac{2 \mu}{B} \partial_{u} \partial_{\mu} P_{\sigma^{\prime}} \partial_{\mu} F_{\sigma}-\frac{m_{\sigma}}{m_{\sigma^{\prime}}} \partial_{u} Q_{\sigma^{\prime}} F_{\sigma}\right),
$$

$\Gamma_{\sigma \sigma^{\prime}}^{\mu}=\frac{\gamma_{\sigma \sigma^{\prime}}}{m_{\sigma}^{2}} \frac{2 \mu}{B}\left[\partial_{u} \partial_{\mu} P_{\sigma^{\prime}} \partial_{u} F_{\sigma}\right.$

$$
\left.+\frac{1}{B}\left(\partial_{\mu} P_{\sigma^{\prime}}+2 \mu \partial_{\mu}^{2} P_{\sigma^{\prime}}\right) \partial_{\mu} F_{\sigma}-\frac{m_{\sigma}}{m_{\sigma^{\prime}}} \partial_{\mu} Q_{\sigma^{\prime}} F_{\sigma}\right],
$$

$\Gamma_{\sigma \sigma^{\prime}}^{\theta}=0$.

Finally, we write the equations that determine the Rosenbluth potentials, (G.7) and (G.8), in the new coordinates,

$$
\begin{array}{r}
\left(\partial_{u}^{2}+\frac{2 \mu}{B} \partial_{\mu}^{2}+\frac{1}{B} \partial_{\mu}\right) P_{\sigma^{\prime}}=Q_{\sigma^{\prime}} \\
\left(\partial_{u}^{2}+\frac{2 \mu}{B} \partial_{\mu}^{2}+\frac{1}{B} \partial_{\mu}\right) Q_{\sigma^{\prime}}=-8 \pi F_{\sigma^{\prime}}
\end{array}
$$

Euratom (C) 2013.

\section{References}

[1] Helander P and Simakov A N 2008 Phys. Rev. Lett. 101145003

[2] Simakov A N and Helander P 2011 Plasma Phys. Control. Fusion 53024005

[3] Mantica P et al 2009 Phys. Rev. Lett. 102175002

[4] Garren D A and Boozer A H 1991 Phys. Fluids B 32822

[5] Simakov A N and Helander P 2009 Phys. Plasmas 16042503

[6] Parra F I and Catto P J 2008 Plasma Phys. Control. Fusion 50065014

[7] Parra F I and Catto P J 2009 Plasma Phys. Control. Fusion 51095008

[8] Parra F I and Catto P J 2010 Plasma Phys. Control. Fusion 52085011

[9] Parra F I, Barnes M, Calvo I and Catto P J 2012 Phys. Plasmas 19056116

[10] Parra F I and Calvo I 2011 Plasma Phys. Control. Fusion 53045001

[11] Parra F I and Catto P J 2010 Plasma Phys. Control. Fusion 52045004

Parra F I and Catto P J 2010 Plasma Phys. Control. Fusion 52059801

[12] Parra F I and Catto P J 2010 Phys. Plasmas 17056106

[13] Parra F I, Barnes M and Catto P J 2011 Nucl. Fusion 51113001

[14] Calvo I and Parra F I 2012 Plasma Phys. Control. Fusion 54115007

[15] Cary J R and Shasharina S G 1997 Phys. Plasmas 43323

[16] Cary J R and Shasharina S G 1997 Phys. Rev. Lett. 78647

[17] Landreman M and Catto P J 2012 Phys. Plasmas 19056103

[18] Helander P and Nührenberg J 2009 Plasma Phys. Control. Fusion $\mathbf{5 1} 055004$

[19] Boozer A H 1983 Phys. Fluids 26496

[20] Nührenberg J and Zille R 1988 Phys. Lett. A 129113

[21] Helander P 2007 Phys. Plasmas 14104501

[22] Sugama H, Watanabe T H, Nunami M and Nishimura S 2011 Phys. Plasmas 18082505 
[23] Reiman A et al 1999 Plasma Phys. Control. Fusion 41 B273

[24] Dimits A M, Williams T J, Byers J A and Cohen B I 1996 Phys. Rev. Lett. 7771

[25] Dorland W, Jenko F, Kotschenreuther M and Rogers B N 2000 Phys. Rev. Lett. 855579

[26] Dannert T and Jenko F 2005 Phys. Plasmas 12072309

[27] Candy J and Waltz R E 2003 J. Comput. Phys. 186545

[28] Chen Y and Parker S E 2003 J. Comput. Phys. 189463

[29] Peeters A G et al 2009 Comput. Phys. Commun. 1802650

[30] Kornilov V, Kleiber R, Hatzky R, Villard L and Jost G 2004 Phys. Plasmas 113196

[31] Xanthopoulos P and Jenko F 2007 Phys. Plasmas 14042501

[32] Baumgaertel J A, Belli E A, Dorland W, Guttenfelder W, Hammett G W, Mikkelsen D R, Rewoldt G, Tang W M and Xanthopoulos P 2011 Phys. Plasmas 18122301

[33] Watanabe T H, Sugama H and Nunami M 2011 Nucl. Fusion 51123003

[34] Catto P J 1978 Plasma Phys. 20719

[35] Krommes J A 2012 Annu. Rev. Fluid Mech. 44175

[36] McKee G R et al 2001 Nucl. Fusion 411235

[37] Braginskii S I 1965 in Reviews of Plasma Physics ed M A Leontovich (New York: Consultants Bureau)

[38] Barnes M, Parra F I and Schekochihin A A 2011 Phys. Rev. Lett. 107115003

[39] Frieman E A and Chen L 1983 Phys. Fluids 25502
[40] Lee X S, Myra J R and Catto P J 1983 Phys. Fluids 26223

[41] Lee W W 1983 Phys. Fluids 26556

[42] Bernstein I B and Catto P J 1985 Phys. Fluids 281342

[43] Dubin D H E, Krommes J A, Oberman C and Lee W W 1983 Phys. Fluids 263524

[44] Hahm T S 1988 Phys. Fluids 312670

[45] Brizard A J and Hahm T S 2007 Rev. Mod. Phys. 79421

[46] Littlejohn R G 1983 J. Plasma Phys. 29111

[47] Dimits A M, LoDestro L L and Dubin D H E 1992 Phys. Fluids B 4274

[48] Dimits A M 2012 Phys. Plasmas 19022504

[49] D'haeseleer W D, Hitchon W N G, Callen J D and Shohet J L 1991 Flux Coordinates and Magnetic Field Structure ed R Glowinski et al (Berlin: Springer)

[50] Chapman S and Cowling T G 1970 The Mathematical Theory of Non-uniform Gases 3rd edn (Cambridge Mathematical Library) (Cambridge: Cambridge University Press)

[51] Sugama H, Okamoto M, Horton W and Wakatani M 1996 Phys. Plasmas 32379

[52] Boozer A H 1981 Phys. Fluids 241999

[53] Connor J W and Hastie R J 1973 Nucl. Fusion 13221

[54] Sugama H, Watanabe T H, Nunami M and Nishimura S 2011 Plasma Phys. Control. Fusion 53024004

[55] Lenard A 1960 Ann. Phys. 10390 